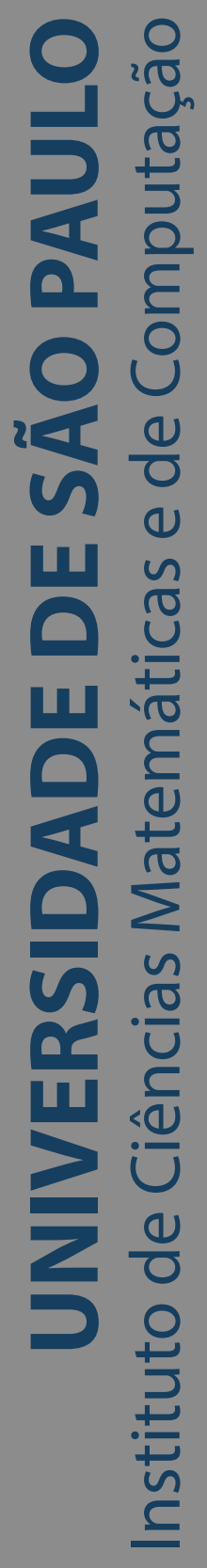

\title{
Visual crime pattern analysis
}

\section{Germain García Zanabria}

Tese de Doutorado do Programa de Pós-Graduação em Ciências de Computação e Matemática Computacional (PPG-CCMC) 

SERVIÇO DE PÓS-GRADUAÇÃO DO ICMC-USP

Data de Depósito:

Assinatura:

\title{
Germain García Zanabria
}

\section{Visual crime pattern analysis}

\begin{abstract}
Thesis submitted to the Instituto de Ciências Matemáticas e de Computação - ICMC-USP - in accordance with the requirements of the Computer and Mathematical Sciences Graduate Program, for the degree of Doctor in Science. FINAL VERSION

Concentration Area: Computer Science and Computational Mathematics
\end{abstract}

Advisor: Prof. Dr. Luis Gustavo Nonato

\section{USP - São Carlos}

February 2021 
Ficha catalográfica elaborada pela Biblioteca Prof. Achille Bassi e Seção Técnica de Informática, ICMC/USP, com os dados inseridos pelo(a) autor(a)

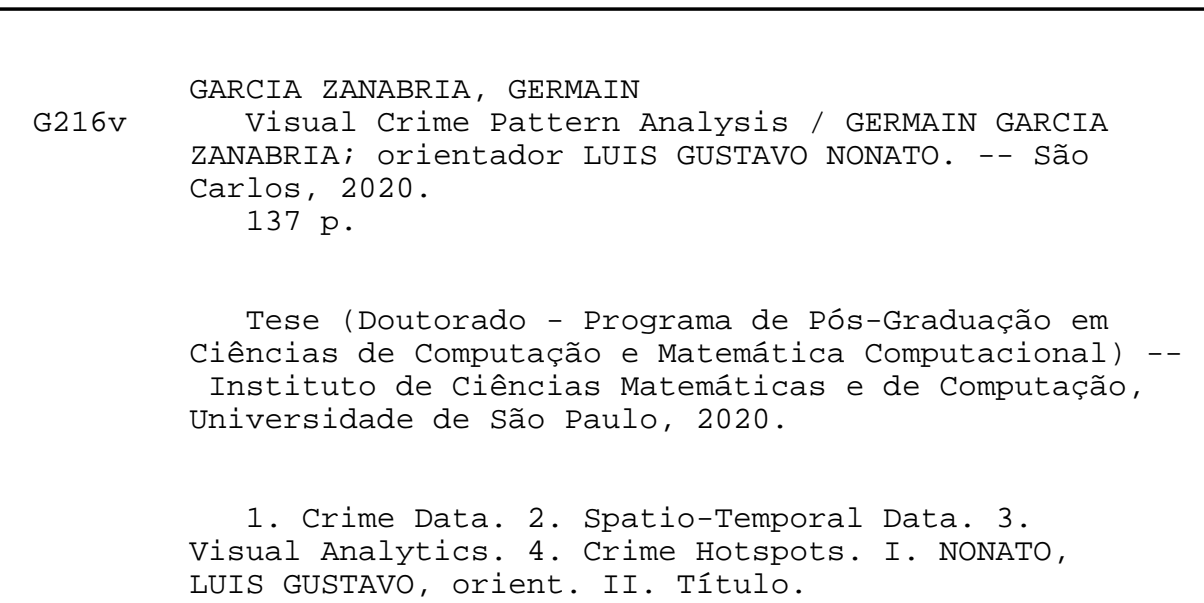

1. Crime Data. 2. Spatio-Temporal Data. 3. Visual Analytics. 4. Crime Hotspots. I. NONATO, LUIS GUSTAVO, orient. II. Título. 


\title{
Germain García Zanabria
}

\section{Análise de padrões criminais}

\author{
Tese apresentada ao Instituto de Ciências \\ Matemáticas e de Computação - ICMC-USP, \\ como parte dos requisitos para obtenção do título \\ de Doutor em Ciências - Ciências de Computação e \\ Matemática Computacional. VERSÃO REVISADA \\ Área de Concentração: Ciências de Computação e \\ Matemática Computacional \\ Orientador: Prof. Dr. Luis Gustavo Nonato
}

USP - São Carlos

Fevereiro de 2021 

A mis primeros maestros

mis padres Grimaldo y Celia.

A mis hermanos Hilarion, Yeny y Kelvin 

First, I would like to thank God!!!.

I want to thank the University of São Paulo (USP) in Brazil and the Instituto de Ciências Matemáticas e de Computação (ICMC) for all these years of education and research.

I would like to thank my parents Grimaldo and Celia, for their support to forge me as a good professional. To my sister and brothers Yeny, Hilarion, and Kelvin, for the motivation throughout the doctorate process. Thank you so much for believing in my capacities, for your support and patience despite the distance.

This thesis would not have been possible without the patience, dedication, and support of my mentor Prof. Dr. Luis Gustavo Nonato. Thanks for believing in me. In this process, I was lucky to have worked with good professionals and friends Afonso Paiva, Claudio Silva, Jorge Poco, Erick Gomez, and Alex Cuadros. All their advice helped me overcome the different problems that arose during the project, from planning to the conclusion of the project. I really appreciate all the effort you put into guiding me.

I would like to thank my friends and collages Jaqueline Silveira, Marcos Raimundo, Filomen Incahuanaco, Laura Cruz, Diego Sintra, and Thalisson Santos. Especially to Jaque, despite many unfavorable situations, we have overcome all of them. Great job, my friend!. I was very lucky to have in my team a good professional and awesome person.

To Prof. Dr. Claudio Silva, Prof. Dr. Juliana Fleire, and Ann Messinger; members of the VIDA lab of Tandon School of Engineering of New York University (NYU). Thanks for the support and advice during the internship. I would also like to thank Raoni Lourenço, Daniela Bertoli, Sonia Castelo, and Roque López for helping me to survive in New York City.

To faculty professors of the ICMC-USP, especially for Maria Cristina Ferreira de Oliveira, Rosane Minghim, Afonso Paiva, Adenilso Simão, and Moacir Ponti. Thanks for sharing your knowledge and experience. It will be always invaluable.

A special thanks to Fundação Amparo à Pesquisa do Estado de São Paulo (FAPESP), processes \#2017/05416 - 1 and \#2019/04434 - 1 for funding my research during my $\mathrm{PhD}$ period and my internship abroad.

To my colleges of Visual and Geometry Processing Group (VPGP) at ICMC-USP Liz Huancapaza, Ivar Vargas, Eric Macedo, Fabio Dias, Lucas Pagliosa, Leonardo Sampaio, Thales Gonçalves, and Evandro. 
To Nucleo de Estudo da Violência of the University of São Paulo (NEV - USP) to provide the data sets used during the doctorate process. Especially to Prof. Dr. Sergio Adorno and Dr. Marcelo Batista Nery, thanks for the feedback and advice during the development of the systems. 
"I have no special talent.

I am only passionately curious."

(Albert Einstein) 



\section{RESUMO}

GARCIA-ZANABRIA, G. Análise de padrões criminais. 2021. 134 p. Tese (Doutorado em Ciências - Ciências de Computação e Matemática Computacional) - Instituto de Ciências Matemáticas e de Computação, Universidade de São Paulo, São Carlos - SP, 2021.

O estudo e análise dos padrões criminais nas grandes cidades é um problema espaço-temporal desafiador. A dificuldade do problema está ligada a diferentes fatores como a modelagem de dados, detecção de hotspots de forma robusta e versátil, análise de padrões espaço-temporais e a delimitação do estudo. Trabalhos anteriores concentraram-se principalmente na análise da criminalidade com o intuito de descobrir padrões associados a fatores sociais, sazonalidade e atividades de rotina urbana em distritos, regiões e bairros inteiros. Portanto, essas ferramentas dificilmente conseguem viabilizar análises de crimes em microescala intimamente relacionadas às oportunidades de crimes, cujo entendimento é fundamental para o planejamento de ações preventivas. Permitir uma análise combinada de padrões espaciais e a visualização dos diferentes padrões de crime ocultos em sua evolução ao longo do tempo é outro desafio enfrentado pela maioria das ferramentas de análise de crime. Nesta tese, propomos um conjunto de abordagens para a análise visual interativa do crime. Com base em métodos de aprendizado de máquina, mecanismos estatísticos e matemáticos e visualização cada metodologia proposta tem como foco problemas específicos de análise de crime. As ferramentas propostas são capazes de explorar locais específicos da cidade o que é essencial para que os especialistas realizarem suas análises de forma detalhada, revelando como características urbanas relacionadas com a mobilidade, comportamento de transeuntes e a infraestrutura das cidades (por exemplo, terminais de transporte público e escolas) podem influenciar a quantidade de algum tipo de atividade criminal. A eficácia e utilidade das metodologias propostas foram demonstradas com um conjunto abrangente de análises quantitativas e qualitativas, bem como estudos de caso executados por especialistas envolvendo dados reais de diferentes cidades. Os experimentos mostram a capacidade das nossas abordagens em identificar diferentes fenômenos relacionados ao crime.

Palavras-chave: Dados Criminais, Mapeamento de Crimes, Dado Spacio-Temporal, Análise Visual, Hotspots de Crimes, Decomposição de Matriz Não-Negativa, Matriz Estocástica . 



\section{ABSTRACT}

GARCIA-ZANABRIA, G. Visual crime pattern analysis. 2021. 134 p. Tese (Doutorado em Ciências - Ciências de Computação e Matemática Computacional) - Instituto de Ciências Matemáticas e de Computação, Universidade de São Paulo, São Carlos - SP, 2021.

Studying and analyzing crime patterns in big cities is a challenging spatio-temporal problem. The difficulty of the problem is linked to different factors such as data modeling, unsophisticated hotspot detection techniques, spatio-temporal patterns, and study delimitation. Previous works have mostly focused on the analysis of crimes with the intent of uncovering patterns associated to social factors, seasonality, and urban activities in whole districts, regions, and neighborhoods. Those tools can hardly allow micro-scale crime analysis closely related to crime opportunity, whose understanding is fundamental for planning preventive actions. Given that, enabling a combined analysis of spatial patterns and the visualization of the different crime patterns hidden in their evolution over time is another challenge faced by most crime analysis tools. In this dissertation, we propose a set of approaches for interactive visual crime analysis. Relying on machine learning methods, statistical and mathematical mechanisms, and visualization, each proposed methodology focus on solving specific crime-related problems. These proposed tools to explore specific locations of the city turned out to be essential for domain experts to accomplish their analysis in a bottom-up fashion, revealing how urban features related to mobility, passerby behavior, and presence of public infrastructures (e.g., terminals of public transportation and schools) can influence the quantity and type of crimes. The effectiveness and usefulness of the proposed methodologies have been demonstrated with a comprehensive set of quantitative and qualitative analyses, as well as case studies performed by domain experts involving real data of different-sized cities. The experiments show the capability of our approaches in identifying different crime-related phenomena.

Keywords: Crime Data, Crime Mapping, Spatio-Temporal Data, Visual Analytics, Crime Hotspots, Non-Negative Matrix Factorization, Stochastic Matrix. 

Figure 1 - Three visual mapping methods . . . . . . . . . . . .

Figure 2 - Hotspot identification improvement. (a) A methodology which uses GDPatterns to crime hotspot study through their related variables, extracted from (WANG et al., 2013a). (b) MSKDE, method to hotspot identification improvement via Marching Squares, extracted from (NETO; SANTOS; VIDAL, 2016). (c) Overview of a web-based tool, temporal view, 3D Hotspot view, 2D spatial view, and spatial slice. . . . . . . . . . . . . . . . . 43

Figure 3 - The COPLINK dashboard . . . . . . . . . . . . . . . . . 44

Figure 4 - VALET screenshot, showing map view to visualize crime. Linked views show the line graph, calendar views, temporal plots, and time slider. . . . . 45

Figure 5 - Web based crime Hotspot mapping techniques . . . . . . . . . . . 46

Figure 6 - HotSketch dashboard. A route has been drawn in the map view window. . . 47

Figure 7 - CrimeVis screen that combine parallel coordinates chart and map with crime events distribution. . . . . . . . . . . . . . . . . . . . 44 47

Figure 8 - SHOC screen showing different hotspots from different periods . . . . . . 48

Figure 9 - Histogram of the $10 \%$ most prevalent crime types, labeling the three most frequent ones, passerby robbery, auto theft, and passerby larceny . . . . . .

Figure 10 - Distribution of crime data from São Paulo over the years, and the quality label of the data, Ouro, Prata, Bronze, Pirita, and Chumbo . . . . . . . . 52

Figure 11 - Histogram of the number of criminal records by crime type. . . . . . . . . 52

Figure 12 - Pipeline overview of the CrimAnalyzer System . . . . . . . . . . . . 59

Figure 13 - a) Region of interest. b) Data matrix containing crime information from the regions in a). Rows correspond to sites while columns are time slices. The darker the color, the closer to zero the number of crimes is. c) Rank $3 \mathrm{NMF}$ decomposition from $X$. d) Rank 5 NMF from $X \ldots$. . . . . . . . .

Figure 14 - (a) Division of São Paulo into 300 groups and (b) SSI distribution in those regions. NMF and $G_{i}^{*}$ detect the same hotspots in most of the cases. . . . .

Figure 15 - Qualitative comparison between NMF and $G_{i}^{*}$. (a) Region where NMF detects more hotspots and (b) region where $G_{i}^{*}$ detects more hotspots. The darkest sites are the ones with high criminality. . . . . . . . . . .

Figure 16 - CrimAnalyzer system: the spatial and temporal interactive views enable the exploration of local regions while revealing their criminal patterns over time. 66 
Figure 17 - Summary of criminal activities and corresponding patterns in four different regions of São Paulo. Crime patterns might change substantially among the regions and also along the time (five years) . . . . . . . . . 70

Figure 18 - Hotspots around the BR116 and SP230 highways considering all crime types. The highways are highlighted in red and the nearby avenues in blue. The ranking type views below show the five most prevalent crimes of each hotspot. 72

Figure 19 - Cargo theft hotspots along two important highways, BR116 and SP230. BR116 presents a much larger and more frequent number of cargo theft than

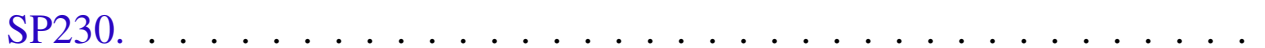

Figure 20 - Commercial establishment burglary tends to increase during the winter (winter in South America goes from mid June to mid September). . . . . . . . .

Figure 21 - The Near Repeat Victimization phenomena. When a home is burgled, the risk of recidivism in a short period of time is not only higher for the targeted home, but also for the nearby homes. . . . . . . . . . . . . . . . .

Figure 22 - Two ways to build a crime-based street-network by closest node based on: (a) Euclidean distance, and (b) edge-node strategy. . . . . . . . . . . . . . .

Figure 23 - An overview of the Mirante tool, a set of spatiotemporal visual resources enabling the exploration of crime patterns in a region: (a) Street-level Heatmap, (b) Temporal Evolution View, (c) Temporal Histogram View, (d) Selector toolbox, (e) Address Search Bar, (f) Evolution Animation Controller, and (g) Local/global crime ruler. . . . . . . . . . . . . . . .

Figure 24 - Street-level heatmap construction in two steps: Color assignation and linear interpolation. . . . . . . . . . . . . . . . . 85

Figure 25 - Two different types of selection of the region of interest. . . . . . . . . 86

Figure 26 - Four months depicted by the evolution animation. . . . . . . . . . . . 87

Figure 27 - Vehicle robbery patterns around São Paulo city: (first-row) overview of Mirante with two hotspots in the street-level heatmap. (second-row) Crime behavior using Temporal Evolution View selecting two periods of time, one from to $1 / 2006$ to $11 / 2010$ and another from $11 / 2010$ to $12 / 2017$. (Third-row) Google Street View images of highlighted nodes in different years and from

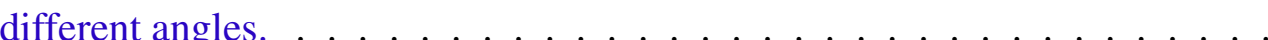

Figure 28 - Urban infrastructure impacting in passerby robbery in the city of São Carlos: (first row) Mirante system with a region of interest. (a), (b), and (c) Street-level Heatmap, Temporal Evolution View with selected time intervals, Temporal Histogram View showing the seasonality of crimes, respectively. . 
Figure 29 - The proposed street-level crime visualization methodology, CriPAV, comprises three main steps. Hotspot Identification: identifying hotspots based on crime intensity and crime probability. Finding Similar Hotspots: hotspot time series embedding (Hotspot2Vec), clustering, and projection into a visual space. Crime X Urban Infrastructure: finding the relation between urban infrastructure and crimes. . . . . . . . . . . . . . . 96

Figure 30 - Data modeling process: (left) Region of study with anchor points (black dots) and criminal events (red dots), (center) approximation of each criminal event to the nearest anchor point, and (right) final street network, each anchor point contains a temporal series. . . . . . . . . . . . . . . . 100

Figure 31 - (a) Probability versus Intensity scatter plot, (b) filtering based on intensity and probability with $\alpha=1$, and (c) filtering based on intensity and probability with $\alpha=0.3$, the red anchor points are the hotspots. Parameter $\alpha$ controls the slope of the separation line. The value of $\alpha$ is the weight one wants to give to intensity of probability when filtering the hotspots. . . . . . . . . . 102

Figure 32 - (1) Region of interest. (2) time series containing information of interest anchor points in (1). (3) filtering in Probability versus Intensity. (4) Hotspots clusterization and LAMP visualization based on hotspot2vec. . . . . . . . . 103

Figure 33 - Autoencoder result: the blue line represent the original time series, and the orange ones are the decoded time series. . . . . . . . . . . . . . . 104

Figure 34 - CriPAV system: Hotspot, spatial, and temporal interactive views enabling the exploration of local regions while revealing their criminal patterns over time. 106

Figure 35 - Analysis of hotspot identification technique. Comparison between missed hotspots with different cut variables. (left) Probability versus Intensity, red dots are hotspots identified by Intensity and Mixed definitions, blue dots are hotspots identified just by Mixed definition, and green dots are points identified only by Intensity Definition. (middle) The time series of blue and green dots. (right) Location View with plotted points. . . . . . . . . . . . 110

Figure 36 - Comparison of Passerby, Commercial Establishment, and Vehicle robberies patterns over the city. (a-first row) Concentration and dispersion tendency of hotspots; (a-second row) Relation between hotspots and socioeconomic variables, and (b) Spatial behavior of hotspots clusters and socioeconomic variables. . . . . . . . . . . . . . . . . . . . . . . . 112

Figure 37 - Hotspots infrastructure comparisons over time. (1) Clusterization and projection of hotspots using Hotspot2Vec, (2) Between-Group Chart showing temporal behavior of each group, (3) time series of the selected group of hotspots (Group 0 and Group 2), and (4) temporal images of point P1, P2, and $\mathrm{P} 3 \ldots \ldots \ldots \ldots \ldots \ldots . \ldots \ldots \ldots$ 



\section{LIST OF ALGORITHMS}

Algorithm 1 - Assigning crimes to vertices. . . . . . . . . . . . . . . . 83 

Table 1 - Classification of relevant related works in crime Analysis. . . . . . . . . . 40

Table 2 - Nomenclature used throughout the manuscript. . . . . . . . . . . . 50

Table 3 - View properties and their analytical tasks (Sec. 4.2.2). . . . . . . . . 65

Table 4 - Methodological and visualization properties and their analytical tasks presented in Sec.5.2.2. . . . . . . . . . . . . . . . . . 85

Table 5 - Goals and their related analytical tasks. . . . . . . . . . . . . 99

Table 6 - Methodological and visualization properties and their related tools. . . . . 106 



\section{LIST OF ABBREVIATIONS AND ACRONYMS}

\begin{tabular}{ll} 
CTC & Crime, Traffic, and Civil \\
DBSCAN & Density-Based Spatial Clustering \\
DCKDE & Dynamic Covariance KDE \\
DTW & Discrete Time Warping \\
FAPESP & Fundação Amparo à Pesquisa do Estado de São Paulo \\
GDP & Gross Domestic Product \\
GDPatterns & Geo-spatial Discriminative Patterns \\
GIS & Geographic Information Systems \\
HDBSCAN & Hierarchical Density-Based Spatial Clustering \\
HOT & Hotspot Optimization Tool \\
ICMC & Instituto de Ciências Matemáticas e de Computação \\
KDE & Kernel Density Estimation \\
LAMP & Local Affine Multidimensional Projection \\
MAUP & Modifiable Areal Unit Problem \\
MSKDE & Marching Squares KDE \\
NEV - USP & Nucleo de Estudo da Violência of the University of São Paulo \\
NKDE & Network-constrained KDE \\
NMF & Non-Negative Matrix Factorization \\
NYU & New York University \\
PAI & Predictive Accuracy Index \\
RTM & Risk Terrain Modeling \\
SOM & Self Organizing Map \\
STAC & Spatial and Temporal Analysis of Crime \\
STKDE & Spatio-Temporal KDE \\
TPD & Tucson Police Department \\
TVCG & IEEE Transactions on Visualization and Computer Graphics \\
US & United States \\
USP & University of São Paulo \\
VALE & Visual Analytics Law Enforcement Toolkit \\
Visual and Geometry Processing Group \\
\hline NPG
\end{tabular}



INTRODUCTION ....................... 29

$1.1 \quad$ Motivation ..................... 29

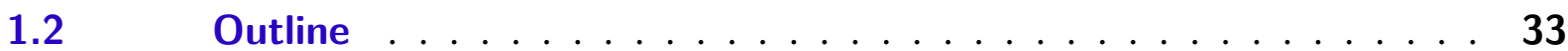

2 CRIME DATA ANALYSIS . . . . . . . . . . . . . 35

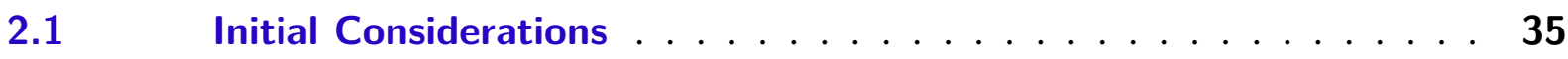

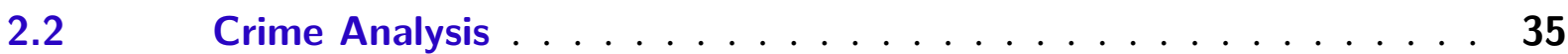

$2.3 \quad$ Hotspot Centered Crime Analysis . . . . . . . . . . . . . 36

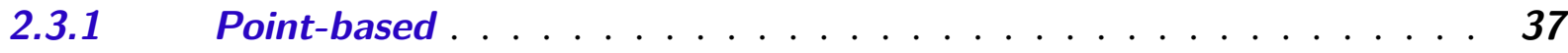

2.3.2 Choropleth Mapping . . . . . . . . . . . . . . . 37

2.3.3 Local Auto-correlation . . . . . . . . . . . . . . . . . 38

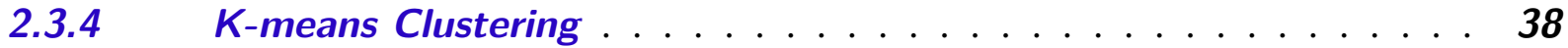

2.3.5 Spatial and Temporal Analysis . . . . . . . . . . . . . . 38

$2.4 \quad$ Environmental Crime Analysis . . . . . . . . . . . . . . . . . 39

2.4.1 Socio-Economic based Crime Analysis . . . . . . . . . . . . . . . . 39

2.4.2 Environmental Infrastructure based Crime Analysis . . . . . . . . . . 41

2.4.3 Social Entities based Crime Analysis . . . . . . . . . . . . . . . 41

$2.5 \quad$ Crime Pattern Visualization . . . . . . . . . . . . . 42

2.5.1 Spatio-temporal Data Visualization . . . . . . . . . . . . . . 42

2.5.2 Street-Level Visualization Techniques . . . . . . . . . . . . 46

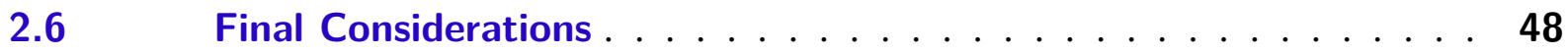

3 DATA SETS AND NOMENCLATURE $\ldots \ldots . \ldots$

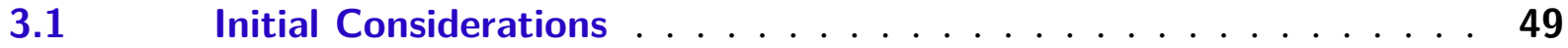

3.2 Setting the Nomenclature . . . . . . . . . . . . . 49

$3.3 \quad$ Data sets . . . . . . . . . . . . . . . . 49

3.3.1 Data Set 1 (2000 - 2006) - São Paulo . . . . . . . . . . . . . . 51

3.3.2 Data Set 2 (2006 - 2017) - São Paulo . . . . . . . . . . . . . . . . . 51

3.3.3 Data Set 3 (2014 - 2019) - São Carlos . . . . . . . . . . . . . . . 52

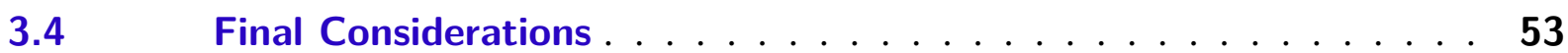

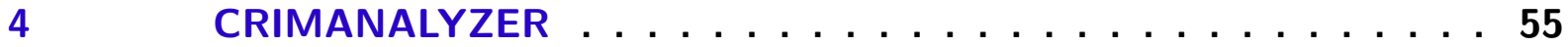

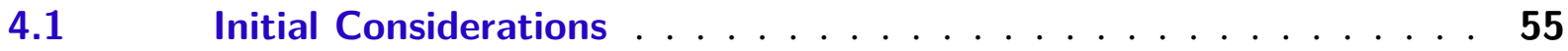

$4.2 \quad$ Challenges and Analytical Tasks .............. 56 
4.2.1 Problem Analysis . . . . . . . . . . . . . . . 56

4.2.2 Analytical Tasks . . . . . . . . . . . . . . . . . 57

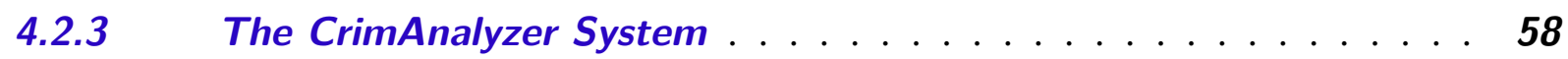

$4.3 \quad$ Hotspot Identification Model . . . . . . . . . . . . . . 59

4.3.1 Non-Negative Matrix Factorization . . . . . . . . . . . 60

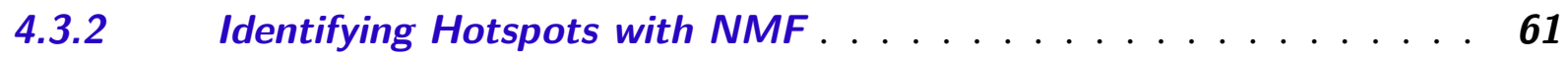

$4.4 \quad$ Visual Design . . . . . . . . . . . . . . . . 65

4.4.1 Control Menu . . . . . . . . . . . . . . . . . . . . 66

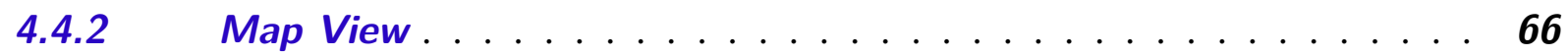

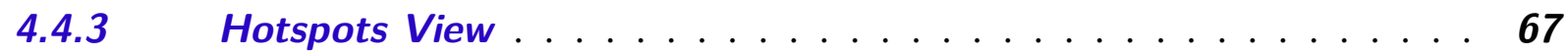

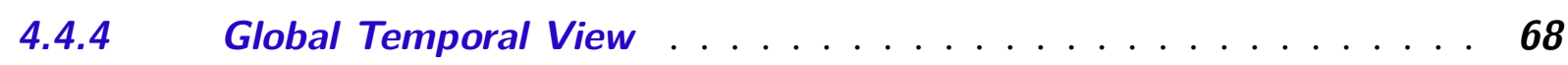

4.4.5 Cumulative Temporal View . . . . . . . . . . . . . . . . 68

4.4.6 Ranking Type View . . . . . . . . . . . . . . . 68

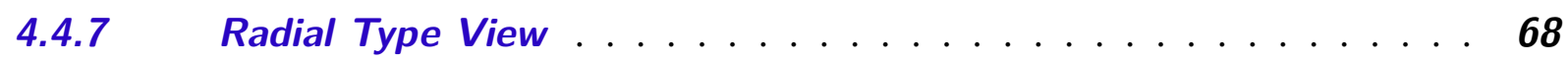

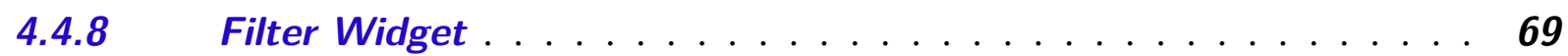

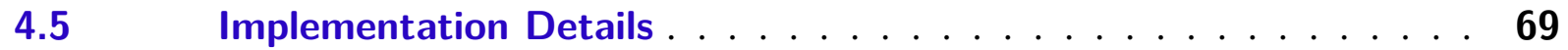

$4.6 \quad$ Case Studies . . . . . . . . . . . . . . . . . . 69

4.6.1 Comparing Crime Patterns over the City $(T 1, T 2, T 3) \ldots \ldots$

4.6.2 Hotspot Analysis and Cargo Theft (T1, T4, T5) . . . . . . . 72

4.6.3 Seasonality and the Temporal Element of Crime (T3) . . . . . . 74

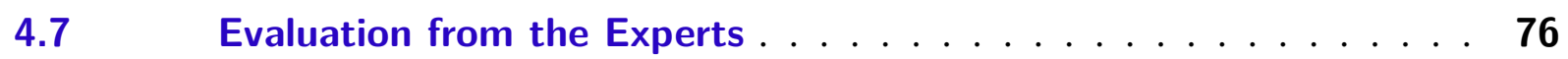

4.8 Discussion and Limitations . . . . . . . . . . . . . . 76

$4.9 \quad$ Final Considerations . . . . . . . . . . . . . . . . 78

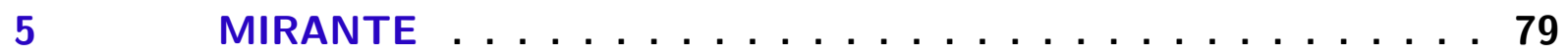

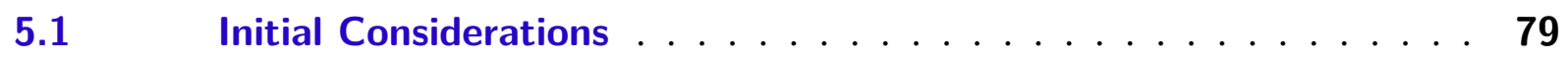

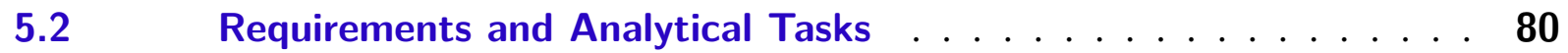

5.2.1 Requirements. . . . . . . . . . . . . . . . . 80

5.2.2 Analytical Tasks . . . . . . . . . . . . . . . . 81

$5.3 \quad$ Mirante System . . . . . . . . . . . . . . . . . . . . . 81

5.3.1 Building the spatial representation . . . . . . . . . . . 82

5.3.2 Assigning data to the nodes of the spatial graph representation . . 82

$5.4 \quad$ Visual Components . . . . . . . . . . . . . . . . . 84

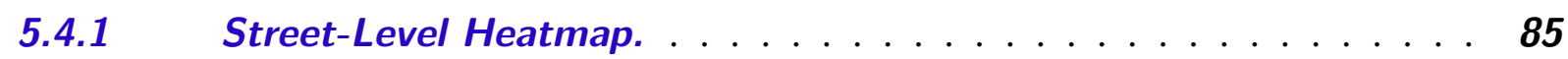

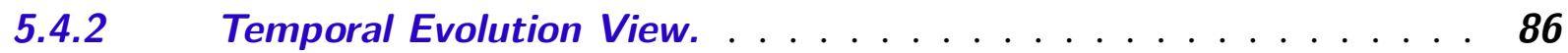

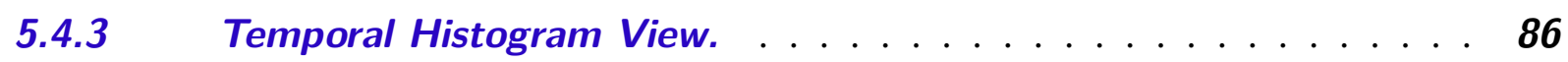

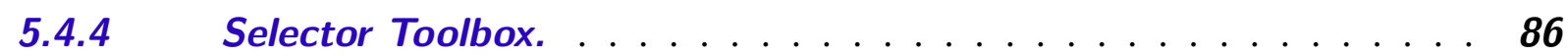

5.4.5 Address Search Bar. . . . . . . . . . . . . . . . . 87

5.4.6 Evolution Animation Controller. . . . . . . . . . . . . 87

5.4.7 Local/Global Crime Ruler. . . . . . . . . . . . . . . . . . 87 


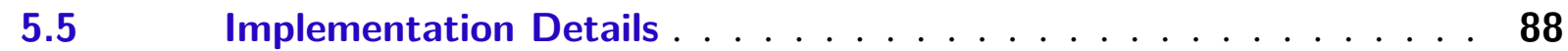

$5.6 \quad$ Case Studies . . . . . . . . . . . . . . . . . 88

5.6.1 Vehicular Robbery in São Paulo City. . . . . . . . . . . . . 88

5.6.2 Passerby Robbery in São Carlos . . . . . . . . . . . . . . . 89

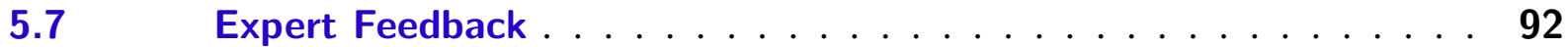

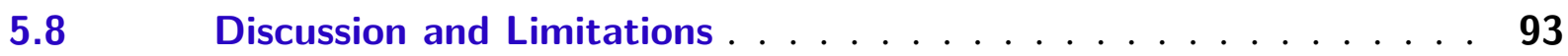

$5.9 \quad$ Final Considerations . . . . . . . . . . . . . . . . . . 94

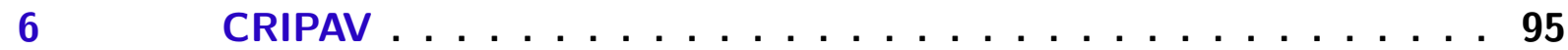

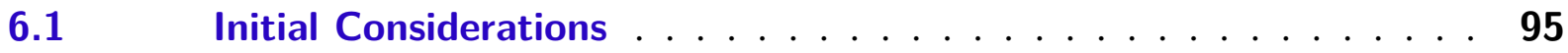

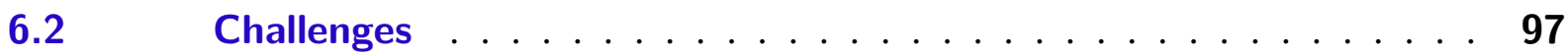

6.2.1 Goals and Analytical Tasks . . . . . . . . . . . . . . . . 97

6.2.2 Analytical Tasks . . . . . . . . . . . . . . . . . 98

6.3 Data Modelling . . . . . . . . . . . . . . . . . 99

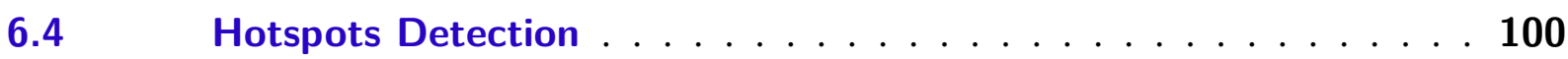

6.4.1 Mathematical Foundation . . . . . . . . . . . . . . . 101

6.4.2 Computing the Stochastic Matrix . . . . . . . . . . . . . . . 101

6.4.3 Selecting Hotspots . . . . . . . . . . . . . . . . . 102

6.4.4 Validation - Selecting Hotspots . . . . . . . . . . . . . . 103

$6.5 \quad$ Finding Similar Hotspots . . . . . . . . . . . . . . . . . . . . 104

6.5.1 Validation - Grouping Similar Hotspots . . . . . . . . . . . . 105

$6.6 \quad$ Visual Components . . . . . . . . . . . . . . . 105

6.6.1 Location view . . . . . . . . . . . . . . . . . . . 106

6.6.2 Hotspot Scatter View (Probability vs Intensity) . . . . . . . . . 107

6.6.3 Crime Pattern Projection . . . . . . . . . . . . . . . . . . 107

6.6.4 Within-Group Joy Chart . . . . . . . . . . . . . . . . 107

6.6.5 Between-Group Chart . . . . . . . . . . . . . . . . . . . . 108

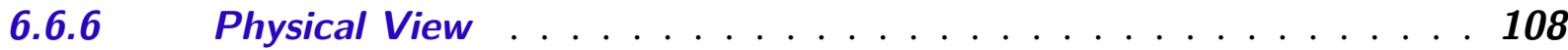

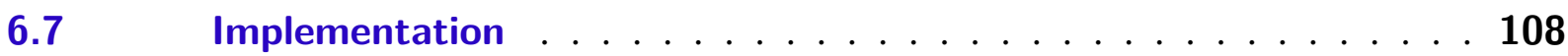

$6.8 \quad$ Case Studies . . . . . . . . . . . . . . . . . . . . . 109

6.8.1 Intensity only versus Intensity \& Probability . . . . . . . . . . . 109

6.8.2 Understanding Crime Patterns Over the City . . . . . . . . . . 111

6.8.3 Relating Urban Infrastructure and Crime Patterns . . . . . . . 113

6.9 Evaluation from Experts . . . . . . . . . . . . . . . 114

6.10 Discusion and Limitations . . . . . . . . . . . . . . 115

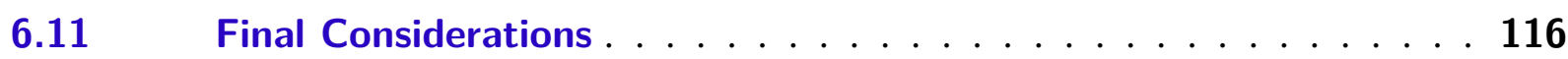

7 CONCLUSION AND FUTURE WORKS $\ldots \ldots \ldots \ldots$

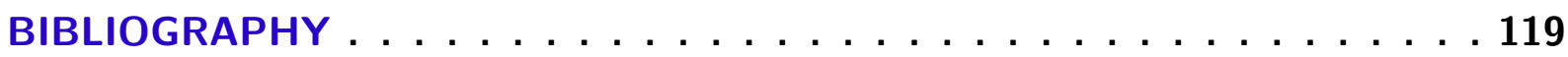


A.1 
CHAPTER

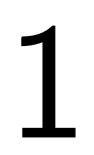

INTRODUCTION

\subsection{Motivation}

Since the mid-1970s, Brazilian society has experienced a transition process from military dictatorship to democracy. With this political transition, it was expected that conflicts would increasingly be solved, reducing the prevalence of violence. That has not happened. In fact, the transition has been accompanied by an explosion of conflicts, many of which were associated with urban crimes (ADORNO, 2013). In the early 1980s, the Brazilian homicide rates were similar to the ones in the United States (US); however, by the end of the decade (1990s), Brazil had already the double homicides when compared to U.S. (CALDEIRA; HOLSTON, 1999). In 2012, the number of homicides in Brazil stood at about four times the U.S. rate (INGRAM; COSTA, 2017). In 2018, fourteen out of the fifty most violent cities in the world were in Brazil (this list does not include cities in war zones, such as Syria or Ukraine) (WOODY, 2018). In 2019, after adopting new policies to fight crime, Brazil's murders rates finally fell, but it still remains among the largest in the world. Besides a large number of lethal violence, Brazil also presents high levels of general crime victimization, similar to other Latin American countries (MURRAY; CERQUEIRA; KAHN, 2013). There is still no consensus among social scientists about the reasons that explain these trends in the evolution of crime and violence in Brazilian society, in particular in the big cities (ADORNO, 2013). Among the explanations that arise more frequently is the exhaustion of traditional security policy models.

Extracting and analyzing crime patterns in urban areas is a challenging spatio-temporal problem. The hardness of the problem is linked to the great variability of patterns among the different types of crimes and the large amount of data involved in such analysis. In recent years, it is undeniable that crimes have not only grown but also become more violent and modernized. In contrast, agencies in charge of law and order (e.g., police and the criminal justice system) have not kept up with these trends. The gap between the dynamics of crime and violence and the state's ability to contain them within the law rule has widened. Therefore, introducing modern 
instruments for the management of public order and crime containment is imperative to make public security policies more efficient in any big city. In this context, the branch of Geographic Information Systems (GIS) called Crime Mapping focuses on the development of tools to explore and analyze the spatio-temporal behavior of crimes, leveraging the importance of urban, social, and environmental characteristics as determinants for crime opportunity (CHEN et al., 2005; CHAINEY; RATCLIFFE, 2013). Current crime mapping tools combine techniques from different fields such as mathematics and statistics (ALVES; RIBEIRO; RODRIGUES, 2018; NONATO; CARMO; SILVA, 2020; VITO et al., 2020), machine learning (Yadav et al., 2017; Joshi; Sabitha; Choudhury, 2017), optimization and visualization (GARCIA-ZANABRIA et al., 2019; SILVA et al., 2017; BADAWY et al., 2018), and social sciences (CANTER; YOUNGS, 2016; SANDERS; CONDON, 2017).

Hotspot analysis has been one of the main resources employed by the public security agencies of most cities to plan police patrolling and design preventive actions (CHAINEY; TOMPSON; UHLIG, 2008). Hotspot identification techniques aim to identify high-risk locations that require more profound attention. Although sophisticated mechanisms have been proposed to detect hotspots (EFTELIOGLU; SHEKHAR; TANG, 2020), the search for a high prevalence of crimes ends up neglecting sites where certain types of crimes are frequent but not sufficiently intense to be considered statistically significant (WANG et al., 2013a), mainly when compared to their surroundings, which can be more harmful to the community than intensive crime waves that occur in a short period of time (WANG et al., 2013a). Moreover, most techniques enable only rudimentary mechanisms to analyze an important component of unlawful activities, the temporal evolution of crimes, and corresponding patterns.

There is another important aspect in the context of crime analysis, the spatial discretization of the urban areas under analysis. The spatial discretization impacts directly the computation and detection of hotspots. Moreover, according to environmental criminology, the concentration and persistence of crimes in certain locations are not random; that is, they occur due to prevalent characteristics present in those locations (MCCULLOCH et al., 2005). Early studies demonstrate that crimes are related to demography (ALVES et al., 2013; ALVES; RIBEIRO; MENDES, 2013), population (GOMEZ-LIEVANO; YOUN; BETTENCOURT, 2012b; OLIVEIRA; BASTOS-FILHO; MENEZES, 2017), socioeconomic level (POVEDA, 2012; KELLY, 2000; LAURITSEN; REZEY; HEIMER, 2014), and unemployment (HOJMAN et al., 2001; HOJMAN, 2002; LEVITT, 2001a) of each location. Therefore, characteristics' changes of particular locations impact crime activity over time, making spatio-temporal hotspot analysis a fundamental task (BRANTINGHAM; BRANTINGHAM, 1998; ECK; WEISBURD, 2015). Crime events are typically examined across many different spatial discretizations, such as states, cities, census blocks, neighborhoods, and blocks. Nevertheless, urban crime activities happen on micro-places (i.e., street segments and corners), which are thus a more meaningful representation of locations than arbitrarily-defined regions. 
Understanding the dynamic of crime patterns over time is another important aspect of crime analysis. Space-time hotspot researches sustain that there is empirical evidence that locations of crime incidents tend to exhibit both spatial as well as temporal concentrations (BRANTINGHAM; BRANTINGHAM, 1998; ECK; WEISBURD, 2015; BLOCK; DAVIS, 1996). Moreover, patterns of spatial and temporal concentration of crime are deciding factors for planning crime prevention measures (JOHNSON et al., 2007). However, most hotspot based analytic tools, mainly the ones in use by security agencies, do not enable resources to identify and group hotspots according to their temporal behavior, hampering the identification of factors that can make crime viable or not over time.

This dissertation presents a compilation of different methodologies for interactive visual crime analysis. Each proposed methodology addresses specific problems in crime analysis. These methodologies have been developed in close collaboration with domain experts and supported by mathematical, computational, and machine learning mechanisms.

In summary, this dissertation presents methodologies, techniques, and results developed during the doctorate period. It compiles the results from the following publications:

- "CrimAnalyzer: Understanding Crime Patterns in São Paulo", (GARCIA-ZANABRIA et $a l ., 2019)$. Published at IEEE Transactions on Visualization and Computer Graphics (TVCG) - 2020.

The main contributions of this work are:

- A new methodology to identify crime hotspots based not only on the number of crimes but also on their variation and recurrence rate.

- A visual analytics machinery that allows users to visually perform spatial and temporal queries towards understanding patterns and temporal dynamics of crimes.

- CrimAnalyzer, a visualization-assisted tool that integrates the analytical machinery in a set of linked views. CrimAnalyzer operates on target spatial regions to uncover relevant information of the region as a whole and also from its individual sites.

- A set of case studies revealing interesting phenomena about the dynamics of crime in São Paulo, supporting hypotheses and theories raised by domain experts and described in the literature.

- "Mirante: Interactive Monitor for Crime Data Analysis", (GARCIA-ZANABRIA et al., 2020a). This paper has got the Best Computer Graphics/Visualization Main Track Paper Award of SIBGRAPI - 2020.

The main contributions of this work are:

- A crime mapping methodology that relies on street maps as spatial representation, which allows the spatio-temporal analysis of large regions as well as specific locations of a city; 
- Mirante, a simple web-based visual analytic tool that provides a number of interactive resources to explore and identify spatio-temporal crime patterns;

- Two case studies based on real data that demonstrate the usefulness of our methodology to reveal interesting crime-related phenomena in large and medium-sized cities in Brazil.

- "CriPAV: Street-Level Crime Patterns Analysis and Visualization", (GARCIA-ZANABRIA et al., 2020b). Submitted to TVCG - 2020.

The main contributions of this work are:

- A new method to identify crime hotspots based not only on the number of crimes but also on their occurrence probability. By combining probability and intensity of crimes in a scatter plot diagram our methodology enables the visual identification of locations where crimes are concentrated as well as sites where crimes are frequent but do not necessarily occur in large amounts.

- A method to create groups of hotspots with similar temporal behavior despite their spatial location. The method relies on a deep learning autoencoder embedding mechanism called Hotspot2Vec.

- CriPAV, a visualization-assisted analytical tool that integrates a set of linked views designed to uncover relevant information about hotspots. CriPAV enables mechanisms to identify, explore, and analyze crime hotspots in a street-level of detail.

- A set of case studies that attest the effectiveness and usefulness of the proposed methodology to reveal interesting phenomena about the dynamics of crime in São Paulo - Brazil.

Additional works developed in the doctorate period:

- Alvarenga Silveira, Jaqueline; Garcia-Zanabria, G.; Paiva Neto, Afonso; Piccirillo, Debora; Batista Nery, Marcelo; Adorno de Abreu, Sergio França; Nonato, Luis Gustavo. Crime Patterns and Urban Infrastructure around São Paulo City Schools: An Analytical Study via Non-Negative Tensor Factorization. In: NETSCI - NETCRIME, 2019, Burlington Vermont. NetCrime, 2019

- Alvarenga Silveira, J., Garcia-Zanabria, G., Paiva A., Piccirillo D.,Batista Nery, M., Adorno S., \& Luis Gustavo Nonato. São Paulo City Schools and Their Surroundings: from Non-Negative Tensor Factorization to Pattern Identification. XL Congresso Nacional de Matemática Aplicada e Computacional. Minisymposium on Mathematics Against Crime, 2019. 
The core of the proposal consists in presenting different methodologies that allow a visual spatio-temporal crime pattern analysis of urban areas considering different characteristics (socio-economic, infrastructure, and social factors). To do this, we have to sort out different problems: hotspot definition and detection, space modeling, and identifying patterns related to the dynamics of crime patterns. Our approaches faced these problems from different fronts: (1) Given the crime events in an urban space, we propose two different methods (depending on the spatial discretization) to identify and present hotspots considering not only the intensity but also the frequency of crimes; (2) street level domain discretization, switching from grid-based to a street-based spatial discretization; (3) Spatio-temporal crime patterns analysis, supported by visualization and machine learning mechanisms to extract and visually present different spatio-temporal patterns. Considering these solutions, we have divided our proposals into three projects that brought three papers listed above (three principal publications). Moreover, for each project, we have presented different qualitative and quantitative experiments with real data from large and mid-sized cities, which have been validated by domain experts.

\subsection{Outline}

This dissertation is organized into three main parts: The first part (Chapter 2) covers concepts and relevant literature about crime analysis to contextualize the problems addressed during the doctorate. The second part (Chapters 3 to 6) presents details about our contributions developed during the $\mathrm{PhD}$ process. Finally, the third part (Chapter 7) presents the conclusions.

In summary, the subjects covered by each chapter are:

- Chapter 2: Review of the Literature describes a set of relevant methodologies to crime analysis organized into three main categories: Hotspot-centered crime analysis techniques, environmental crime analysis, and visualization techniques as the core of crime analysis.

- Chapter 3: Data sets and Nomenclature presents the data used in our experiments. Moreover, this chapter sets up the terminology that will be important to make the reading easier.

- Chapter 4: CrimAnalyzer, a visualization assisted analytic tool that allows users to analyze the behavior of crimes in specific regions of the city. CrimAnalyzer allows users to identify local hotspots and the pattern of crimes associated with them while still showing how hotspots and corresponding crime patterns change over time.

- Chapter 5: Mirante, a crime mapping visualization system that allows spatio-temporal analysis of crime patterns in a street-level of detail. Mirante addresses a street-level analysis following three stages: Selection of a region of study, region modeling as a graph, and crime visualization. In the visualization, the edges are colored depending on the criminality, highlighting the most dangerous streets. 
- Chapter 6: CriPAV (Crime Pattern Analysis and Visualization), a street-level visualizationassisted analytical tool that integrates a set of linked views designed to uncover relevant information about crime hotspots. It is a new methodology to identify crime hotspots based not only on intensity but also on the probability of occurrence, a hotspot grouping technique based on the similarity of crime time series.

- Chapter 7: Conclusion summarizes our contributions, discusses the limitations of our approaches, and point future research directions. 
CHAPTER

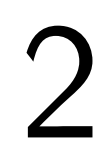

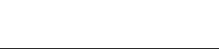

\section{CRIME DATA ANALYSIS}

\subsection{Initial Considerations}

Crime analysis refers to systematic and analytical processes that provide timely and pertinent information about crime patterns and trends to assist users (i.e., authorities, and experts) in crime comprehension, reduction, prevention, and evaluation (EMIG; HECK; KRAVITZ, 1980; FELSON; BOBA, 2010). This chapter provides the literature about crime data analysis from different perspectives. We organize the related work into three main categories (not exclusive) according to their focus of study. In the first category (Section 2.3 ), we list a set of hotspotcentered crime analysis techniques. The second category (Section 2.4) describes a set of works that focus on environmental crime analysis. Finally, the third category (Section 2.5) presents works that use visualization techniques as the core mechanism to crime analysis. The division of the techniques is presented in Table 1, and it must be clear that there is considerable overlap between those categories.

\subsection{Crime Analysis}

Crime can be defined as breaking or breaching criminal law (penal code) that governs a particular geographical area (jurisdiction) aimed at protecting the lives, property, and the right of citizens in that jurisdiction (AKPINAR, 2005). In other words, crime is an offense against a person, or his/her property, violation of socially accepted rules of human ethical or moral behavior.

In order to help law enforcement agencies deploy resources in a more effective manner to prevent, control, and reduce criminal activities, it is necessary to identify and analyze their patterns. In this context, crime analysis plays an essential role in enabling methodologies and tools to analyze criminal activities from different perspectives. 
The main focus of crime analysis is the study of crime type (e.g., rape, robbery, and burglary), discover their patterns (e.g., seasonality, and spatial crime type variation), and related traits such as the nature of incidents, offenders, victims or targets (SANTOS, 2016). Although many different characteristics are relevant in crime analysis, there are three key features that are essential: socio-demographic, spatial, and temporal attributes. Socio-demographic information consists of the features of individuals and groups such as sex, race, income, age, and education. Spatial information includes geographic characteristics such as latitudes, longitudes, streets, and blocks. Finally, the temporal information consists of chronological information such as hour and date.

The literature of crime analysis is extensive, ranging from mathematical to computational mechanisms such as data mining (CHEN et al., 2004; YING, 2016), optimization (WANG et al., 2013a), machine learning (WANG et al., 2013b; Yadav et al., 2017), statistics (OSGOOD, 2010), and data visualization (GAO et al., 2014; XU; CHEN, 2005). In order to better contextualize our contributions we divided these works into three main categories.

\subsection{Hotspot Centered Crime Analysis}

Hotspot analysis is a ubiquitous technique used to visualize where the crime tends to be the highest. This method is commonly used by law enforcement, police, and crime reduction agencies to aid in the decision-making and determine where to target and deploy resources. Actually, crime hotspot analysis has been one of the main resources employed by public security agencies of most cities to plan police patrolling and design preventive actions (CHAINEY; TOMPSON; UHLIG, 2008). Existing hotspot based techniques can be divided into six categories: Kernel Density Estimation (KDE) (BAILEY; GATRELL, 1995; MCLAFFERTY; WILLIAMSON; MCGUIRE, 2000; JOHNSON; BOWERS et al., 2008; ANDERSON, 2009), point-based (CHAINEY; RATCLIFFE, 2013), choropleth mapping (HIRSCHFIELD; BOWERS, 2014), local auto-correlation (MENCKEN; BARNETT, 1999; RATCLIFFE; MCCULLAGH, 1999; CRAGLIA; HAINING; WILES, 2000), k-means clustering (GRUBESIC; MURRAY, 2001; MOHD; HERMAN; SHARIF, 2017), and Spatial and Temporal Analysis of Crime (STAC) (BLOCK, 1995; LEVINE, 2017).

Kernel Density Estimation (KDE). It is based on crime incidents aggregation within a userspecified search radius; after that, it is calculated a continuous surface that represents the density of crimes across the desired area. The smooth surface map shows the variation of crime density across the area (see Figure 1 (right)). In other words, KDE generalizes or "smooths" discrete crime events positions (data points) in a continuous surface area (BAILEY; GATRELL, 1995). However, as pointed out by Hart and Zandbergen (2014), properly setting the parameters of a KDE is not easy. A loose choice of parameters can lead to erroneous or inaccurate results that overestimate or disregard hotspot location (CHAINEY; REID; STUART, 2002; KALINIC; 
Figure 1 - Three ways of hotspots visual representation. (Left) discrete point mapping, extracted from (VASCONCELOS; PINHO, 2017). (Center) choropleth mapping, extracted from (VASCONCELOS; PINHO, 2017). (Rigth) Kernel Density Estimation.
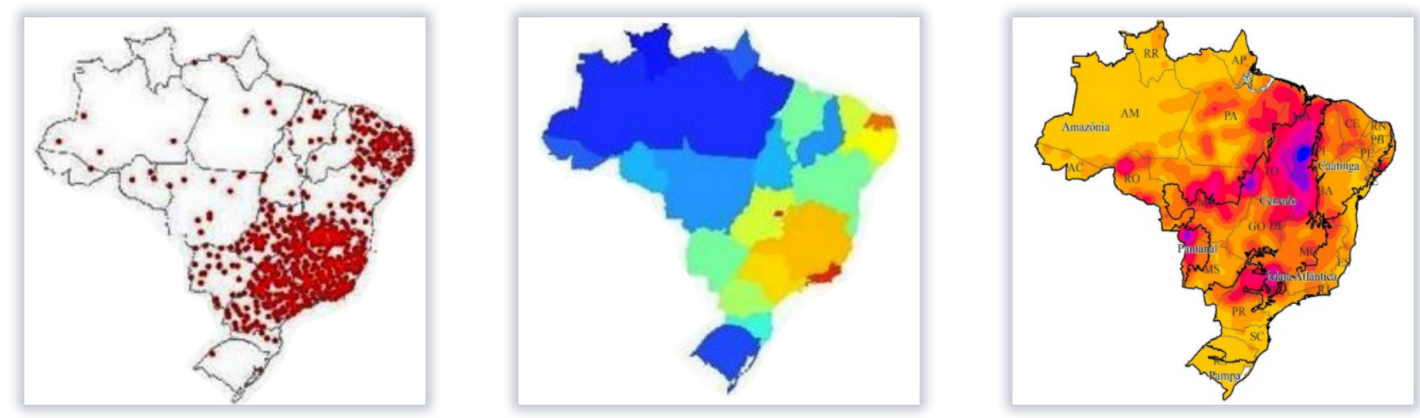

Source: MORAIS, SILVA and ROCHA (2014).

KRISP, 2018). Another issue with KDE based techniques is that locations presenting regular, but not intense, criminal activities are hardly pointed out as hotspots.

Later works improve some drawbacks proposing some variations of $\mathrm{KDE}$, such as Marching Squares KDE (MSKDE) (NETO; SANTOS; VIDAL, 2016), Network-constrained KDE (NKDE) (NIE et al., 2015), and Spatio-Temporal KDE (STKDE) (HU et al., 2018). Dulce, Ramírez-Amaya and Riascos (2018) developed a Self-Exciting point process model to predict crime and present partial results of its deployment on field in Bogotá - Colombia. For that, they use KDE in the process based on the model proposed in (MOHLER et al., 2011). Table 1 (first row) shows a list of works based on KDE.

\subsubsection{Point-based}

The most common method for displaying crime information is by point mapping, consisting of a point representing a crime incident into a map (CHAINEY; RATCLIFFE, 2005). The conglomeration of points is considered a hotspot. An example of point mapping is shown in the Figure 1 (Left); each point represents a Dengue case in Brazil. Table 1 (second row) shows a list of works based on Point mapping.

The main drawback of point mapping methods is the difficulty of identifying the location, relative scale, size, and shape of hotspots when crime incidents are presented as points. The large volume of data makes it difficult to visualize and interpret accurate patterns in the incidents' spatial distribution. Also, certain locations on the map appear to be a single incident point, but maybe multiple events mapped on top of each other.

\subsubsection{Choropleth Mapping}

It is a popular hotspot representation method in which geographic boundary areas (i.e., census blocks, and uniform grids) are used as the basic mapping elements (HIRSCHFIELD; BOWERS, 2014). Unlike point mapping, choropleth mapping uses aggregate data removing 
spatial details. Also, identified hotspots are restricted to the geometrical shape to describe the area of interest. Figure 1 (Center) shows a choropleth representation of Dengue cases in Brazil, considering the states as the unity areas. Table 1 (third row) shows a list of works based on Choropleth mapping.

Choropleth mapping has some limitations; for instance, the distribution of crime events is static at the scale of the map; no inference can be made at a more detailed level. Moreover, the geometric units or boundary areas may not have much to do with crime events (the crime rate probably has more to do with social characteristics than with the state administrative boundaries).

\subsubsection{Local Auto-correlation}

Spatial auto-correlation can be loosely defined as the coincidence of value similarity with location similarity. In other words, high or low values for a random variable tend to cluster in space (positive spatial auto-correlation), or locations tend to be surrounded with very dissimilar values (negative spatial auto-correlation) (ANSELIN; BERA, 1998). Spatial auto-correlation is present when a value for variable $X$ at location $j$ is dependent upon the value of variable $X$ at location $i$ (MENCKEN; BARNETT, 1999) - high or low values of criminality tend to be clustered in space.

The visual representation could be as choropleth mapping techniques. Besides sharing the drawbacks of choropleth mapping, auto-correlation techniques are particularly problematic for dependent variables in regression analysis (LAND; DEANE, 1992). Moreover, negative spatial auto-correlation (very dissimilar values) implies a checkerboard pattern of values and does not always have a meaningful, substantive interpretation (ULLAH, 1998). Table 1 (four row) shows a list of works based on Local auto-correlation.

\subsubsection{K-means Clustering}

This group of techniques use k-means clustering algorithm (KRISHNA; MURTY, 1999) to identify similar spatial groups based on data points (SIRINGI; MALA; RAWAT, 2020) or images (MOHD; HERMAN; SHARIF, 2017).

However, the ability to identify the appropriate number of clusters for a given set of crime events is one of the most fundamental shortcomings of k-means in hotspot detection (GRUBESIC; MURRAY, 2001). Although there are numerous methods outlined in statistics literature for detecting an appropriate number of clusters, the result could be variable depending on the stopping criterion. Table 1 (fifth row) shows works based on k-means Clustering.

\subsubsection{Spatial and Temporal Analysis}

STAC identifies high-intensity areas of crimes based on the spatial and temporal concentrations. It is widely used partly because of simplicity, and it is readily available through a 
comprehensive crime analysis tool called CrimeStat (LEVINE, 2017). Table 1 (sixth row) shows works based on STAC.

However, the critical issue of these techniques is the spatial and temporal discretization. For instance, different spatial discretization can produce different results and lead to erroneous conclusions. Recent works have adopted some strategies such as the search-window approach to carry out street-level hotspot detection to improve the analysis (SHIODE, 2011; SHIODE; SHIODE, 2013; SHIODE et al., 2015).

\subsection{Environmental Crime Analysis}

According to environmental criminology, the concentration and persistence of crimes in certain locations are not random; that is, they occur due to prevalent characteristics present in those locations (MCCULLOCH et al., 2005). Consequently, changes in the characteristics of particular locations impact crime activity over time (BRANTINGHAM; BRANTINGHAM, 1998; ECK; WEISBURD, 2015). In this section, we present works that analyze crimes from an environmental criminology point of view. We group methods into three classes: Socio-economic, infrastructure, and social entities variables.

\subsubsection{Socio-Economic based Crime Analysis}

This group of methods aims to understand the relation between crime events and socioeconomic indicators such as demographics (ALVES et al., 2013; ALVES; RIBEIRO; MENDES, 2013), population (GOMEZ-LIEVANO; YOUN; BETTENCOURT, 2012a; OLIVEIRA; BASTOSFILHO; MENEZES, 2017; OSGOOD, 2000), economic level (POVEDA, 2012; KELLY, 2000; LAURITSEN; REZEY; HEIMER, 2014), and unemployment (HOJMAN, 2004; HOJMAN, 2002; LEVITT, 2001b). For instance, Gomez-Lievano, Youn and Bettencourt (2012a) build a self-consistent statistical framework that characterizes the joint probability distributions of urban indicators and city population sizes across an urban system. To develop this framework, they use urban indicators in Brazil, Colombia, and Mexico. They use these data to derive the conditional probability of the number of homicides per year given the population. Kelly (2000) analyzed the relation between inequality and crime in urban counties in the United States and demonstrates that socially disadvantaged people commit most violent crimes. This study concluded that the most underprivileged members of society who lived in areas of high inequality faced greater pressure and incentives to commit violent crimes. Oliveira, Bastos-Filho and Menezes (2017) developed a methodology to characterize crime concentration dividing cities into regions with the same population size. They confirmed that crime concentrates regardless of city and revealed that the level of concentration does not scale with city size. In particular, they showed that thefts tend to concentrate more than robberies and robberies more than burglaries. Poveda (2012) studied socio-economic and violent crime in seven cities, showing that cities' economic deprivation 
Table 1 - Classification of relevant related works in crime Analysis.

\begin{tabular}{|c|c|c|}
\hline Category & Class & Method \\
\hline \multirow{21}{*}{$\begin{array}{l}\text { Hotspot } \\
\text { Centered }\end{array}$} & \multirow{7}{*}{$\mathrm{KDE}$} & (BAILEY; GATRELL, 1995) \\
\hline & & (MCLAFFERTY; WILLIAMSON; MCGUIRE, 2000) \\
\hline & & (JOHNSON; BOWERS et al., 2008) \\
\hline & & MSKDE (NETO; SANTOS; VIDAL, 2016; NETO et al., 2020) \\
\hline & & NKDE (NIE et al., 2015) \\
\hline & & STKDE (HU et al., 2018), DCKDE (MALIK et al., 2014) \\
\hline & & (DULCE; RAMÍREZ-AMAYA; RIASCOS, 2018) \\
\hline & \multirow[t]{2}{*}{ Point-based } & $\begin{array}{c}\text { (CHAINEY; RATCLIFFE, 2013; CHAINEY; RATCLIFFE, 2005) } \\
\text { (MCEWEN; TAXMAN, 1995) }\end{array}$ \\
\hline & & (HIRSCHFIELD; BOWERS, 2014) \\
\hline & \multirow{2}{*}{ Choropleth mapping } & (BRIMICOMBE et al., 2001) \\
\hline & & CrimAnalyzer (GARCIA-ZANABRIA et al., 2019) \\
\hline & \multirow{4}{*}{ Local auto-correlation } & (MENCKEN; BARNETT, 1999) \\
\hline & & (RATCLIFFE; MCCULLAGH, 1999) \\
\hline & & (CRAGLIA; HAINING; WILES, 2000) \\
\hline & & (SOLTANI; ASKARI, 2017) \\
\hline & \multirow{3}{*}{ K-means clustering } & (GRUBESIC; MURRAY, 2001) \\
\hline & & (SIRINGI; MALA; RAWAT, 2020) \\
\hline & & (MOHD; HERMAN; SHARIF, 2017) \\
\hline & \multirow{3}{*}{$\begin{array}{l}\text { Spatio-Temporal } \\
\text { analysis }\end{array}$} & STAC-NT (SHIODE, 2011; SHIODE; SHIODE, 2013; SHIODE et al., 2015) \\
\hline & & CriPav (GARCIA-ZANABRIA et al., 2020b) \\
\hline & & Mirante (GARCIA-ZANABRIA et al., 2020a) \\
\hline
\end{tabular}

\begin{tabular}{|c|c|c|}
\hline \multirow{12}{*}{$\begin{array}{l}\text { Environmental } \\
\text { Analysis }\end{array}$} & \multirow{4}{*}{ Socio-Economic } & $\begin{array}{l}\text { Population and Demographics (ALVES et al., 2013; ALVES; RIBEIRO; } \\
\text { MENDES, 2013; GOMEZ-LIEVANO; YOUN; BETTENCOURT, 2012a; } \\
\text { OLIVEIRA; BASTOS-FILHO; MENEZES, 2017; OSGOOD, 2000) }\end{array}$ \\
\hline & & $\begin{array}{l}\text { Economic level (POVEDA, 2012; KELLY, 2000; LAURITSEN; REZEY; } \\
\text { HEIMER, 2014) }\end{array}$ \\
\hline & & Unemployment (HOJMAN, 2004; HOJMAN, 2002; LEVITT, 2001b) \\
\hline & & Socio-Economic, infrastructure and population (TensorAnalyzer) \\
\hline & \multirow{4}{*}{ Infraestructure } & RTM (COZENS; SAVILLE; HILLIER, 2005a), (CAPLAN; KENNEDY, 2010) \\
\hline & & $\begin{array}{l}\text { liquor stores (GRUENEWALD et al., 2006; LIVINGSTON, 2010; GRUBESIC; } \\
\text { PRIDEMORE, 2011; DAY et al., 2012) }\end{array}$ \\
\hline & & Subway stations (MCCORD; RATCLIFFE, 2009) \\
\hline & & Parks (DEMOTTO; DAVIES, 2006) \\
\hline & \multirow{4}{*}{ Social Entities } & $\begin{array}{l}\text { (BENNETT; HOLLOWAY; FARRINGTON, 2006; BENNETT; HOLLOWAY; } \\
\text { FARRINGTON, 2009) }\end{array}$ \\
\hline & & (PATTAVINA; BYRNE; GARCIA, 2006) \\
\hline & & $($ GRAAN, 2016) \\
\hline & & (BRUNTON-SMITH; BULLOCK, 2019) \\
\hline \multirow{17}{*}{$\begin{array}{l}\text { Crime Pattern } \\
\text { Visualization }\end{array}$} & \multirow{9}{*}{$\begin{array}{l}\text { Spatio-temporal } \\
\text { Visualization }\end{array}$} & COPLINK (CHEN et al., 2003) \\
\hline & & VALET (MALIK et al., 2010) \\
\hline & & Web_hotspot (ZHOU; LIN; ZHENG, 2012) \\
\hline & & HotSketch (GODWIN; STASKO, 2017) \\
\hline & & CrimeVis (SILVA et al., 2017) \\
\hline & & CrimeWatcher (NETO et al., 2020) \\
\hline & & CrimAnalyzer (GARCIA-ZANABRIA et al., 2019), \\
\hline & & CriPav (GARCIA-ZANABRIA et al., 2020b), \\
\hline & & Mirante (GARCIA-ZANABRIA et al., 2020a), HOT (WANG et al., 2013a) \\
\hline & \multirow{8}{*}{$\begin{array}{l}\text { Street-Level } \\
\text { Visualization }\end{array}$} & CriPav (GARCIA-ZANABRIA et al., 2020b) \\
\hline & & Mirante (GARCIA-ZANABRIA et al., 2020a) \\
\hline & & Street segment crime density (SPICER et al., 2016) \\
\hline & & VitalVizor (Zeng; Ye, 2018), (FENG et al., 2020) \\
\hline & & Trajgraph (Huang et al., 2016) \\
\hline & & VitalVizor (Zeng; Ye, 2018), PHAR (JUNIOR et al., 2019) \\
\hline & & StreetExplorer (SHAO et al., 2016), (DAVIES; JOHNSON, 2015) \\
\hline & & STAC-NT (SHIODE, 2011; SHIODE; SHIODE, 2013; SHIODE et al., 2015) \\
\hline
\end{tabular}

Source: Elaborated by the author. 
and high population density are strong factors in homicide rates. Additionally, they found that economic growth, inequality, poverty, and human capital had a negative influence on violent crimes.

Hojman (2004) proposes an interesting example in our context studying inequality, unemployment, and crime in Latin American cities considering the diversity among cities and using regression analysis to indicate the role of poverty and inequality as causes of crime. Finally, in a Brazilian Context; Alves et al. (2013) studied the relationship between homicide and urban factors with crime data in Brazilian cities. Their study found that Gross Domestic Product (GDP), GDP per capita, income, and male population have positive correlations with homicide. In contrast, child labor, elderly population, female population, illiteracy, sanitation, and unemployment have a negative correlation with homicide.

\subsubsection{Environmental Infrastructure based Crime Analysis}

Although socio-demographic, spatial, and temporal attributes are essential information for crime analysis, some studies move toward the analysis of physical characteristics of individual crime scenes. Caplan and Kennedy (2010) propose Risk Terrain Modeling (RTM) as a methodology to analyze criminality based on the dynamic interaction among social, physical, and behavioral factors that occur in particular places. Risk is defined as the likelihood of an event occur given what is known about the correlation between that event and social and urban factors. Terrain is a grid discretizing the study area where values of risk exist. Modeling broadly refers to the abstraction of the real world at certain places. In this context, some works find correlations between criminality and liquor stores (GRUENEWALD et al., 2006; LIVINGSTON, 2010; GRUBESIC; PRIDEMORE, 2011; DAY et al., 2012), subway stations (MCCORD; RATCLIFFE, 2009), and parks (DEMOTTO; DAVIES, 2006). Cozens, Saville and Hillier (2005a) divide crime factors related to physical characteristics into six categories: surveillance, territoriality, activity support, access control, target hardening, and image maintenance factor.

Image maintenance promotes a positive image and routinely maintaining the built environment to ensure that the physical environment transmits positive signals to all users. Physical incivilities such as damaged buildings, graffiti, and garbage are associated with high crime rates (COZENS; SAVILLE; HILLIER, 2005a). The techniques described above have a common characteristic; namely, they fail to reflect how physical characteristics evolve over time and how environmental changes impact crime dynamics.

\subsubsection{Social Entities based Crime Analysis}

Social organization (i.e., neighborhood, block, apartment, home, and community watch) is a way to promote greater involvement of citizens in the prevention of crime. Some studies show that neighborhood watch programs are generally effective in helping to prevent crime in different 
contexts (BENNETT; HOLLOWAY; FARRINGTON, 2009; GRAAN, 2016; PATTAVINA; BYRNE; GARCIA, 2006).

Bennett, Holloway and Farrington (2006) and Bennett, Holloway and Farrington (2009) present two systematic reviews about the effectiveness of neighborhood watch programs. They found favorable effects, and the same effect is repeated across all presented studies. For instance, Bennett, Holloway and Farrington (2009) found that neighborhood watch was associated with a relative reduction in crime between 16 and 26 percent. In the same vein, a recent work proposed by Graan (2016) focuses on the role and contribution of communities as an effective vehicle of crime prevention in Roodekrans city (Roodepoort - South Africa). They presented a general framework for the growth of successful multi-sector community-based crime prevention initiatives.

Intuitively, citizen involvement in crime programs can be useful in preventing crimes such as house robbery, residential burglary, and vehicle theft. However, Pattavina, Byrne and Garcia (2006) revealed that citizen involvement in collective crime prevention does indeed vary according to each neighborhood, where high-risk neighborhoods demonstrate higher rates of citizens' involvement than low- to moderate-risk neighborhoods.

\subsection{Crime Pattern Visualization}

As detailed above, most crime analysis methods aim to analyze data based on statistics, data mining, mathematics, machine learning, etc. However, in the literature, some methods use visualization as the main tool to visually explore high volumes of crime data. These works use visual analysis to represent simple patterns (WANG et al., 2013a; ROBINSON; CARNES; ORESKOVIC, 2016; MELO; MATIAS; ANDRESEN, 2015; BREETZKE; PEARSON, 2014; YE et al., 2015; NATH, 2006; DERYOL et al., 2016; GAO et al., 2014; KIM et al., 2018; SPICER et al., 2016) to combine different visualization techniques in more sophisticated visual analytic tools (CHEN et al., 2003; MALIK et al., 2010; RAZIP et al., 2014; GODWIN; STASKO, 2017; CALHOUN et al., 2008; SILVA et al., 2017; GARCIA-ZANABRIA et al., 2019; GARCIAZANABRIA et al., 2020a; GARCIA-ZANABRIA et al., 2020b; NETO et al., 2020).

\subsubsection{Spatio-temporal Data Visualization}

Spatio-temporal crime pattern identification and representation is the most popular visual analytic mechanism (YE et al., 2015; NATH, 2006; MELO; MATIAS; ANDRESEN, 2015; GAO et al., 2014). Hotspot identification being a central component, Wang et al. (2013a) introduce a spatial data mining methodology that relies on Geo-spatial Discriminative Patterns (GDPatterns) to capture differences between hotspots and regular areas. They developed a model called Hotspot Optimization Tool (HOT) to improve crime hotspots identification, whose complete pipeline is illustrated in the Figure 2 (a). Other methods aim to improve visual hotspot representation based 
Figure 2 - Hotspot identification improvement. (a) A methodology which uses GDPatterns to crime hotspot study through their related variables, extracted from (WANG et al., 2013a). (b) MSKDE, method to hotspot identification improvement via Marching Squares, extracted from (NETO; SANTOS; VIDAL, 2016). (c) Overview of a web-based tool, temporal view, 3D Hotspot view, 2D spatial view, and spatial slice.

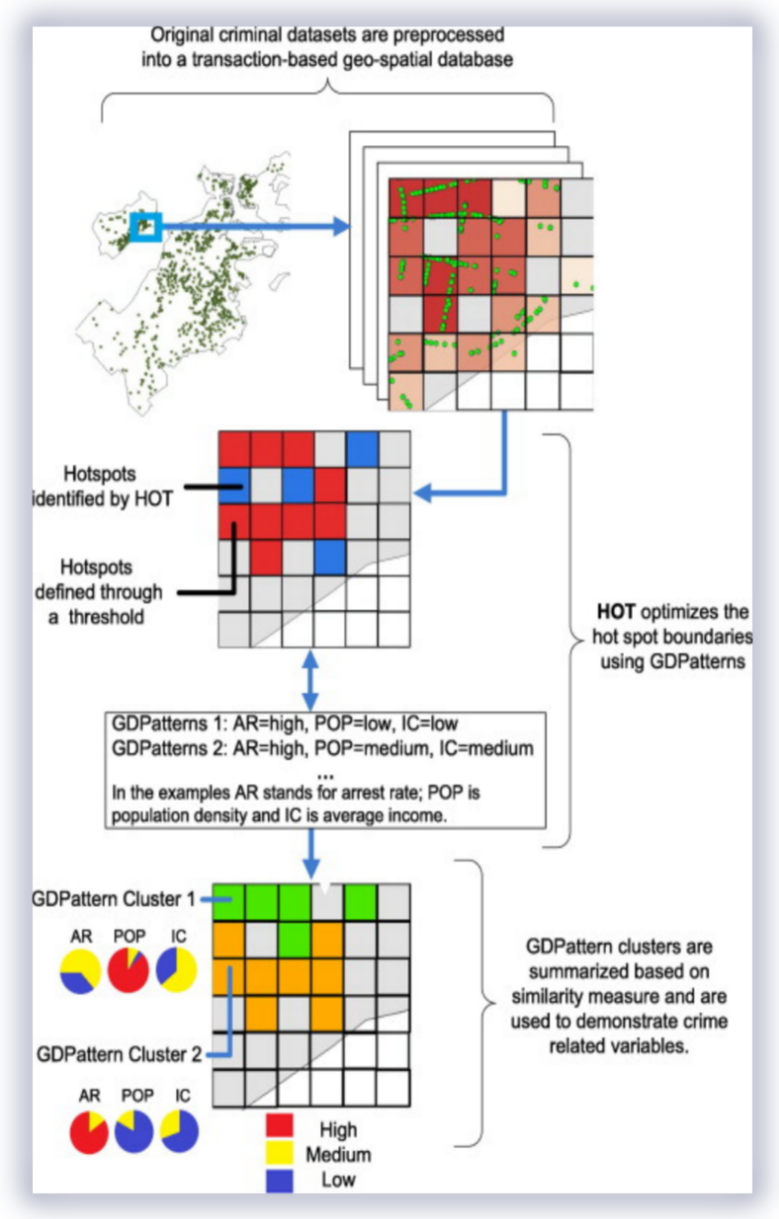

(a) GDPattern

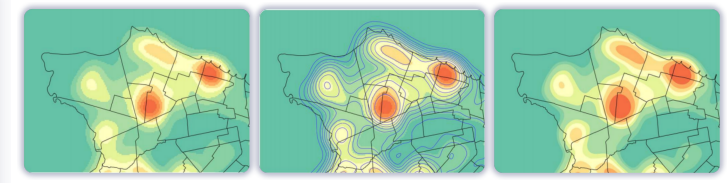

(b) MSKDE

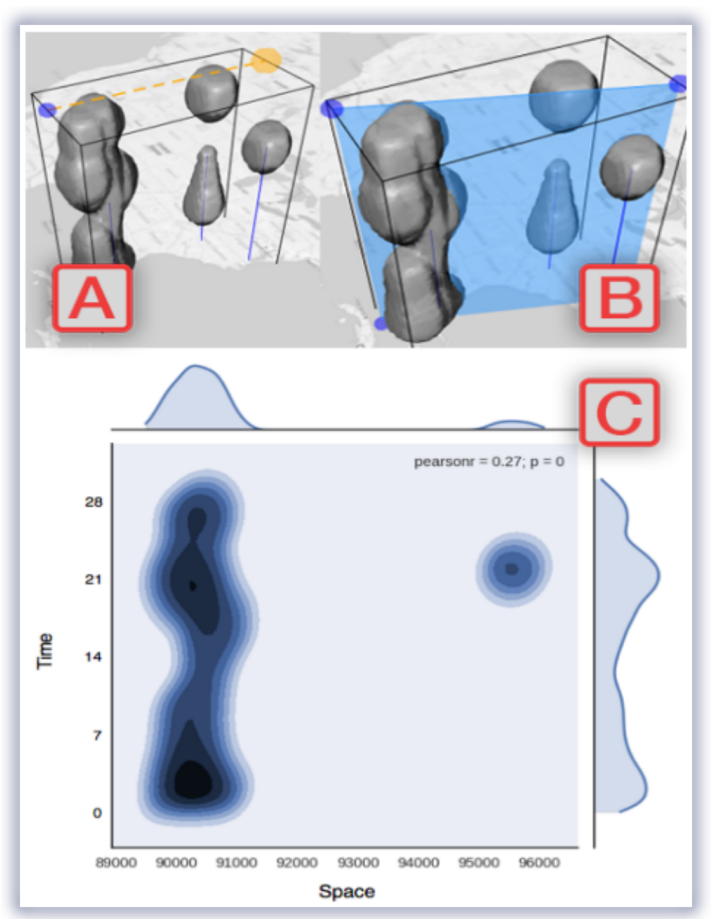

(c) Hotpost and Spatial View

Source: Wang et al. (2013a), Neto, Santos and Vidal (2016), Eaglin, Cho and Ribarsky (2017).

on marching squares (NETO; SANTOS; VIDAL, 2016; NETO et al., 2020) and 3D visualization (EAGLIN; CHO; RIBARSKY, 2017), as illustrated in Figure 2 (b) and Figure 2 (c) respectively.

Chen et al. (2003), in collaboration with the Tucson Police Department (TPD) proposed a system called COPLINK, a user-friendly information system that integrates data from various sources to enable law enforcement agencies with effective information. COPLINK includes spatio-temporal crime analysis using Self Organizing Map (SOM), and various visualization techniques (Hyperbolic Tree, timeline, GIS, and Hierarchical List). Figure 3 shows a screenshot of COPLINK.

Malik et al. (2010) proposed Visual Analytics Law Enforcement Toolkit (VALET) using KDE to help police to better analyze crime data. VALET enables tools to interactively visualize and analyze historical Crime, Traffic, and Civil (CTC) incidents in different geo-spatial and 
Figure 3 - The COPLINK dashboard: representation of a search result using Hyperbolic Tree and Hierarchical List View.

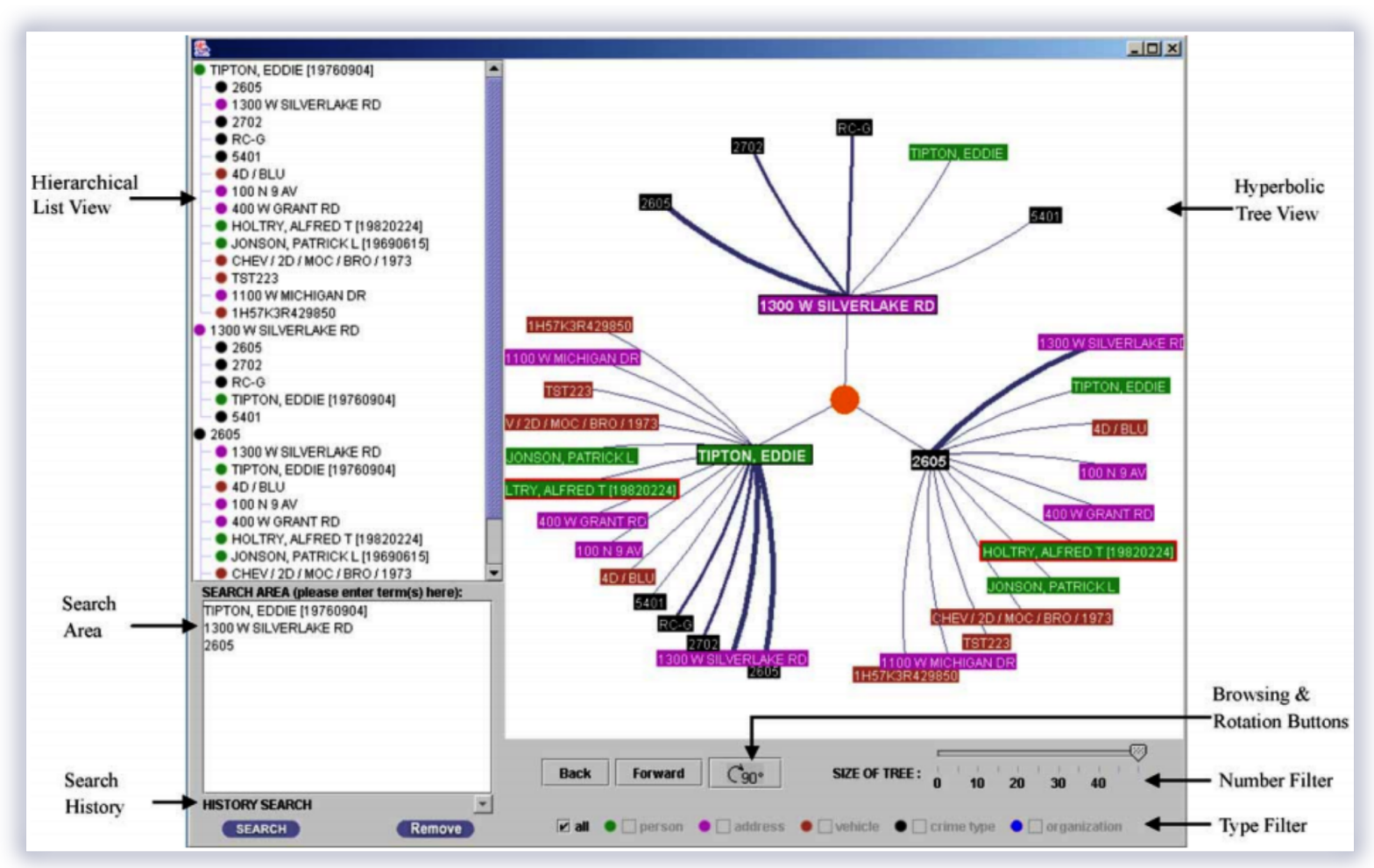

Source: Xiang et al. (2005).

temporal locations. Figure 4 shows a snapshot including linked views with temporal and spatial visualization. VALET has additional characteristics such as mobile visual analysis (RAZIP et al., 2014) and Dynamic Covariance KDE (DCKDE) (MALIK et al., 2014) to highlight areas where future incidents may occur.

Zhou, Lin and Zheng (2012) designed and implanted a simple Web-based GIS methodology for crime mapping and decision support. They present four hotspot techniques: choropleth, grid, spatial ellipse, and kernel density mapping to represent crime hotspots, predict the location of future crime, and optimize crime reduction efforts. Figure 5 shows examples of the four hotspot techniques produced by Zhou, Lin and Zheng (2012).

The use of sketch-based visualization has been proposed for dynamic route planning (GODWIN; STASKO, 2017), combining interactive spatio-temporal hotspots and route planning. The major components are: (1) a sketch-based method for dynamic route planning, which allows police to fast establish a route across the city and discover the number and types of crime along the route, and (2) a spatio-temporal hotspot method considering time, location, season, and crime volume. Figure 6 shows the system interface and its characteristics.

Other approaches present different crime mapping system considering the effectiveness of a wider range of visualization techniques to understand spatio-temporal trends (BRUNSDON; 
Figure 4 - VALET screenshot, showing map view to visualize crime. Linked views show the line graph, calendar views, temporal plots, and time slider.

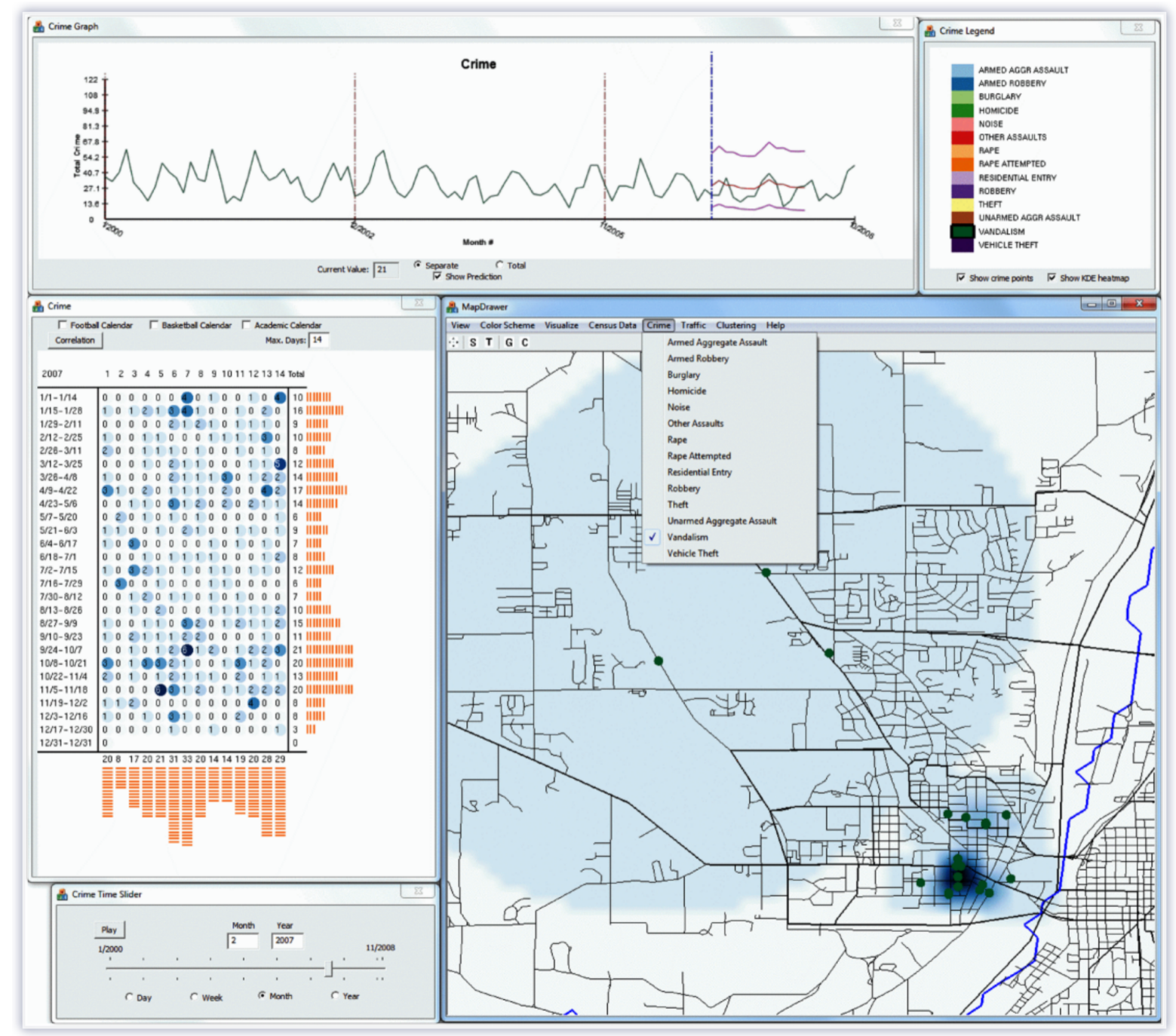

Source: Malik et al. (2010).

CORCORAN; HIGGS, 2007). For instance, Maciejewski et al. (2009), Nakaya and Yano (2010) and Hu et al. (2018) present crime hotspots maps based on KDE and its variations (STKDE). These systems use linked views to represent spatio-temporal data, using geo-map, choropleth map, line chart, Predictive Accuracy Index (PAI) curve, Space-time cube, and heatmap metaphors.

In a Brazilian context, Silva et al. (2017) proposed a simple interactive tool called CrimeVis that allows visualizing multidimensional, spatio-temporal, and multivariate data in an integrated brushing and linking environment. CrimeVis enables the analysis of criminal events distribution over a certain period of time, as well as their social implications. Figure 7 shows a screen of CrimeVis combining parallel coordinates chart and map with crime events distribution.

Another recent work in a Brazilian context was proposed by Neto et al. (2020). They propose CrimeWatcher, a visualization tool that strives for simplicity and ease of use in helping 
Figure 5 - Hotspot mapping techniques. Crime Hotspot Mapping System user interface and (a) discrete point mapping, (b) choropleth mapping, (c) grid thematic mapping, (d) spatial ellipse mapping, and (e) kernel density mapping.

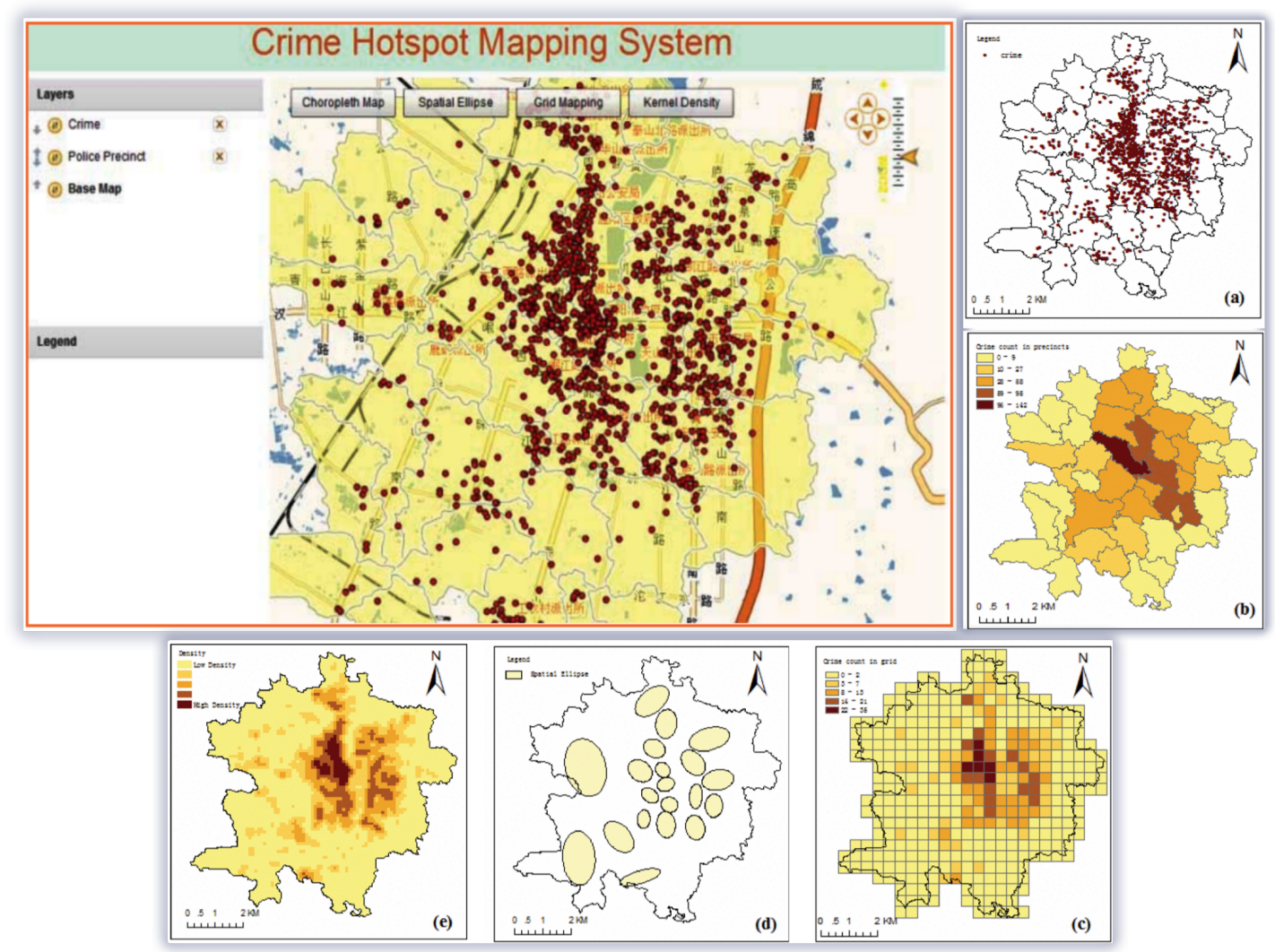

Source: Zhou, Lin and Zheng (2012).

users to perform the analytical tasks. SHOC presents different crime visualizations, such as point maps, choropleth maps, and density maps (KDE and MSKDE). Figure 8 shows an screenshot of $S H O C$ with different hotspots from different periods.

\subsubsection{Street-Level Visualization Techniques}

In addition to spatio-temporal visualization, fine-grained analysis, as the street level ones are also fundamental in the context of crime mapping, as crimes usually take place in street segments (JEAN, 2007; WEISBURD; MORRIS; GROFF, 2009; BRAGA; PAPACHRISTOS; HUREAU, 2010), and they tend to recur in the same or nearby locations (GROFF; WEISBURD; YANG, 2010), such as bars and bus stations (BLOCK; BLOCK, 1995; BLOCK; DAVIS, 1996). Some visualization methods have been developed to enable a street-level analysis of different phenomena, most of which relying on mathematical and computational tools such as complex networks (SPADON et al., 2018; FERREIRA; RUBIANO; MOJICA-NAVA, 2018; PORTA; CRUCITTI; LATORA, 2006), neural networks (YE et al., 2019; LAW; NEIRA, 2019), and clustering (BAK; OMER; SCHRECK, 2010) to enable meaningful visualizations. For instance, StreetExplorer (SHAO et al., 2016) uses line segment enhancing to search for patterns in urban 
Figure 6 - HotSketch dashboard. A route has been drawn in the map view window. Category panel (upper right) shows the crime types filtered based on the route. And additional visualizations show seasonal crime distribution along the route.

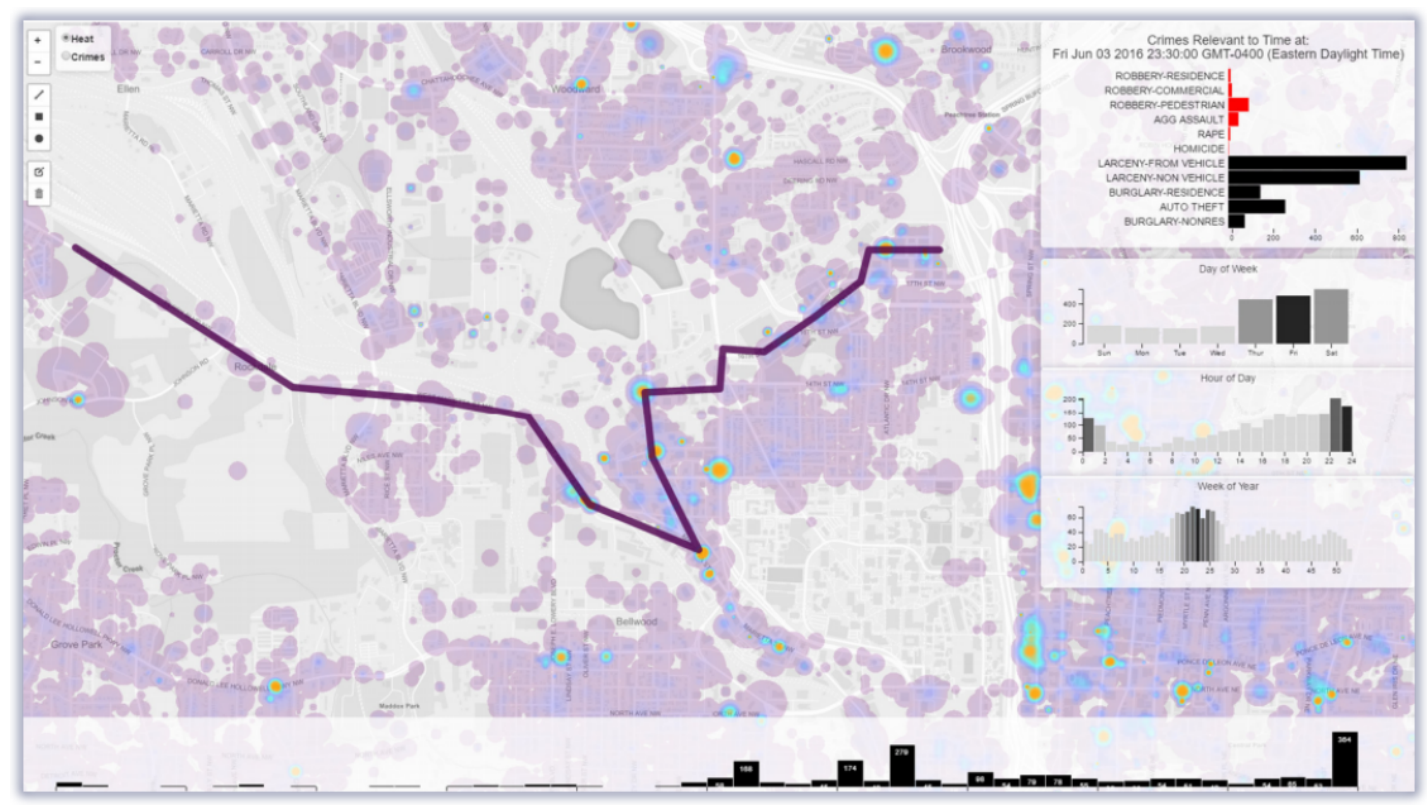

Source: Godwin and Stasko (2017).

Figure 7 - CrimeVis screen that combine parallel coordinates chart and map with crime events distribution.

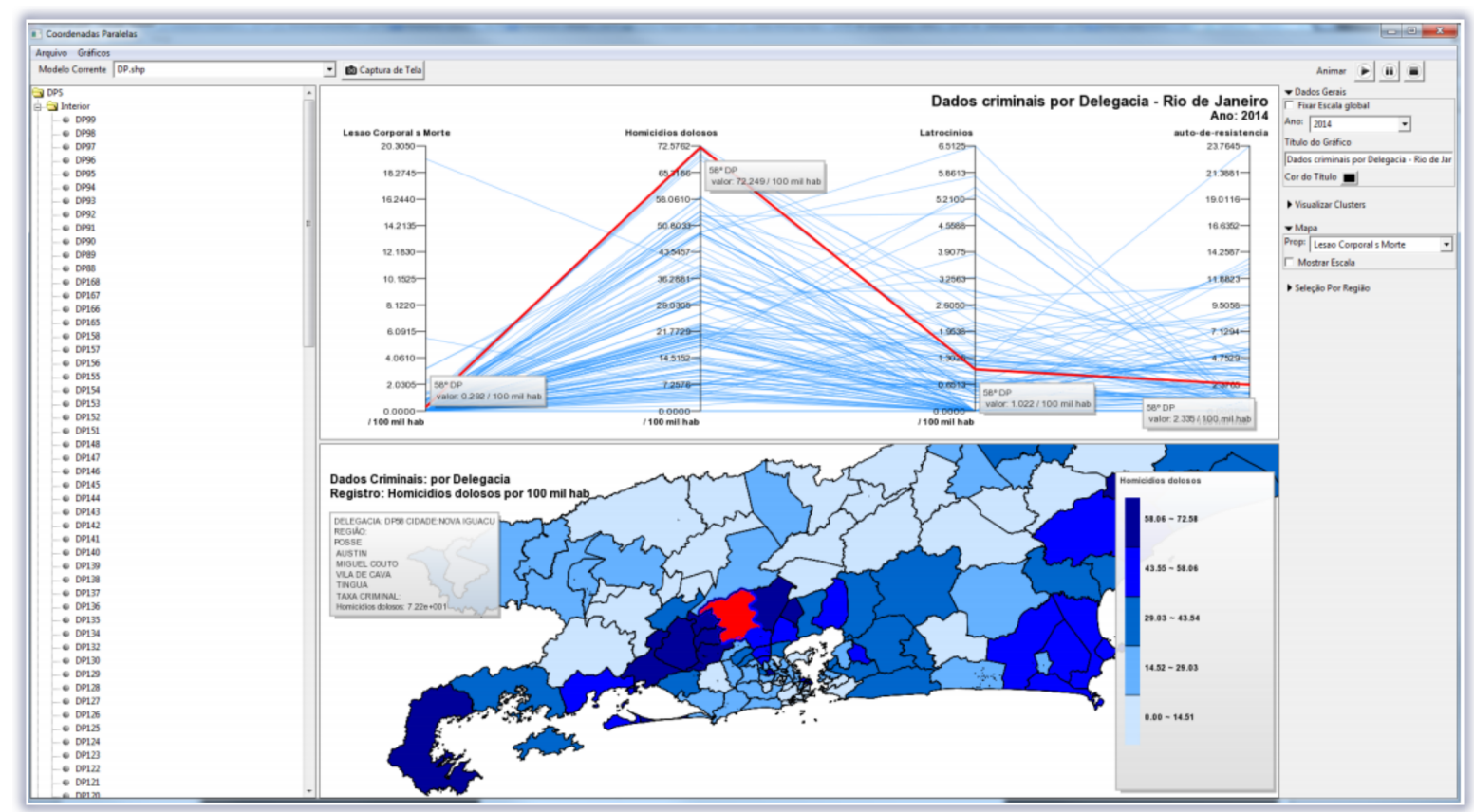

Source: Silva et al. (2017).

street networks. VitalVizor (Zeng; Ye, 2018) combines geometric entities such as streets, blocks, and buildings with parallel coordinates and tree diagrams to understand urban vitality. Wang et al. (2014) relies on animation to explore sparse traffic trajectory data to visualize the movement of vehicles and extract flow patterns locally. Trajgraph (Huang et al., 2016) integrates a node- 
Figure 8 - SHOC screen showing different hotspots from different periods.

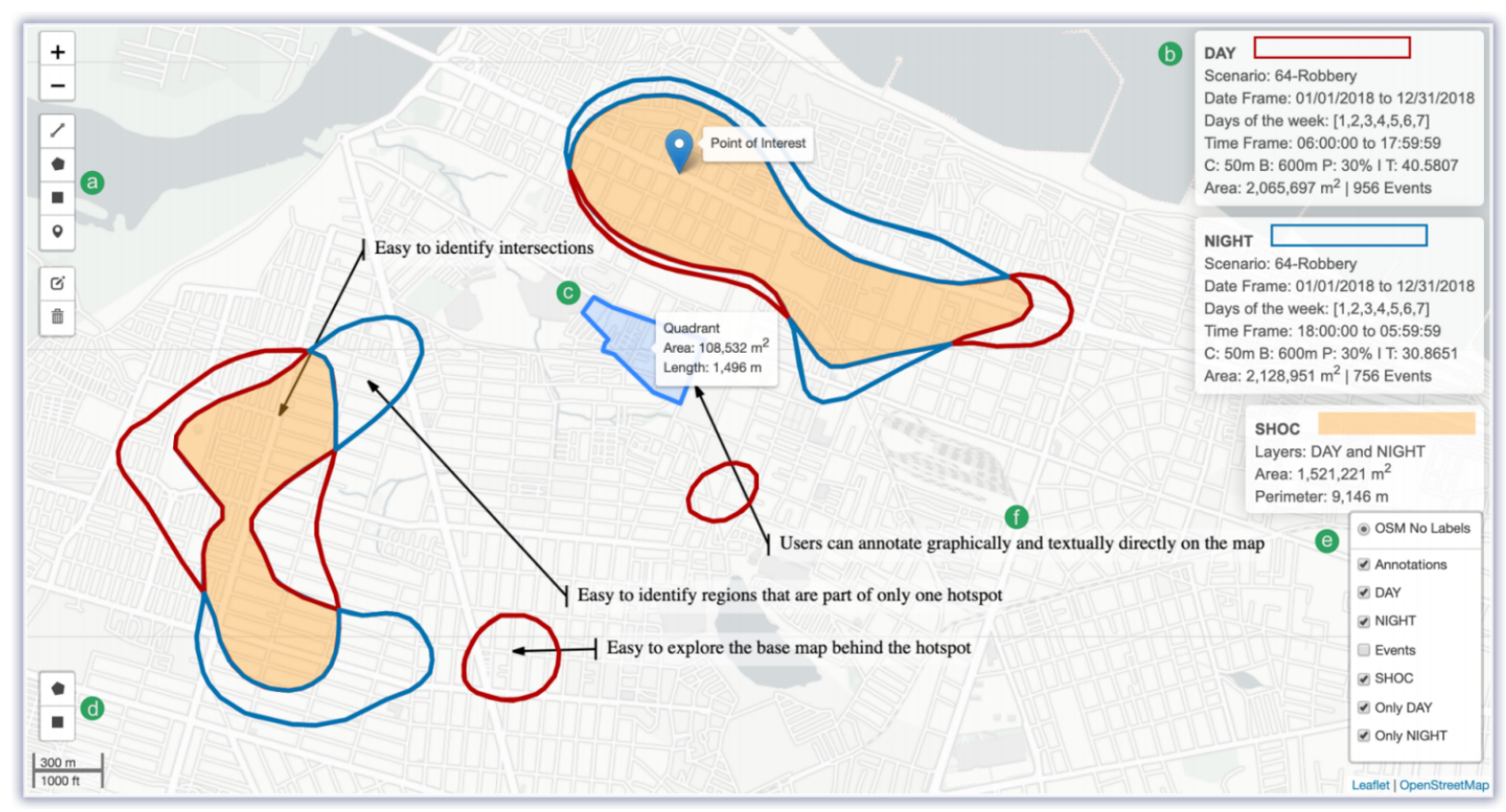

Source: Neto et al. (2020).

link graph view with a street-level map view for understanding urban mobility patterns. Graph measurements, such as PageRank, betweenness, and closeness, are implemented to assist the analysis.

\subsection{Final Considerations}

In this chapter, we summarize basic concepts and related works about crime analysis. These concepts and works are fundamental for understanding our proposals presented in the following chapters.

Although several reviewed tools bear sophisticated analytical mechanisms while relying on different types of data, they have some drawbacks to combine the data from different categories and detecting hotspots. Moreover, most of the existing methods do not use state-of-the-art visual analytic tools, performing their analysis mainly through tables and simple plots. Our approaches propose visualization methodologies that combine information from different categories in an informative visual analytic tool.

From Chapter 4 to Chapter 6, we present the details about our proposals designed in close collaborations with domain experts. 
CHAPTER

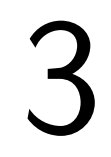

(1)

\section{DATA SETS AND NOMENCLATURE}

\subsection{Initial Considerations}

During the last four years, we have interacted with two social sciences experts whose research focuses on crime analysis. One of the sociologists is a well-known researcher in the study of violence in South America. The other sociologist is an expert in public safety and social sciences applied to urban issues, with a GIS background and vast experience in spatio-temporal crime analysis. In partnership with the police department of the state of São Paulo, the team of experts built three data sets (detailed in Section 3.3.1, Section 3.3.2, and Section 3.3.3). This chapter presents the data sets used to design our proposals, but first, we need to set up terminology that will be important to make the reading easier.

\subsection{Setting the Nomenclature}

Aiming at a fully comprehensible understanding of challenges and problems involved in our study, the specificity of the data, and the methodologies, we carefully settle some definitions that we use throughout the manuscript. Table 2 presents the terms and their definitions.

\subsection{Data sets}

In this work, we analyze crime incidents in two different Brazilian cities with very different characteristics: São Paulo, the largest city in South America, and São Carlos, a mediumsized city in the interior of the state of São Paulo.

São Paulo has an estimated 12,252,023 inhabitants and a demographic density of $7,398.26 \mathrm{inhab} / \mathrm{km}^{2}$. On the other hand, São Carlos has a population of 251,983 inhabitants and a demographic density of 195.15 inhab $/ \mathrm{km}^{2}$, according to the Brazilian Institute of Geography 
and Statistics in 2019. It hosts two major public universities; thus, 30 thousand out of the 220 thousand inhabitants are university students.

Table 2 - Nomenclature used throughout the manuscript.

\begin{tabular}{|c|c|}
\hline Name & Definition \\
\hline Anchor Point & $\begin{array}{l}\text { It is the smallest unit of study in a street network. In our context, anchor points } \\
\text { are the intersections of streets. }\end{array}$ \\
\hline Site & $\begin{array}{l}\text { It is the smallest territorial unity given in the spatial discretization. In our } \\
\text { context, the sites are defined as the census units, each containing from } 250 \text { to } \\
350 \text { residents and/or commercial establishments. }\end{array}$ \\
\hline Region & $\begin{array}{l}\text { It is a set of sites, which can correspond to a whole neighborhood, a particular } \\
\text { portion of a neighborhood, or even a group of sites adjacent to a street or avenue. } \\
\text { It can be defined automatically or by the user. }\end{array}$ \\
\hline Time Unit & $\begin{array}{l}\text { It is the time scale that the temporal data is aggregated, for example, months or } \\
\text { weeks. }\end{array}$ \\
\hline Crime Time Series & $\begin{array}{l}\text { It is the temporal evolution of crimes in each particular location. More specifi- } \\
\text { cally, it is a time series discretized in time units associated to each anchor point } \\
\text { or site. }\end{array}$ \\
\hline Crime Type & It refers to the nature of the crime activity, typified according to the victim. \\
\hline Hotspots & $\begin{array}{l}\text { They are sites or anchor points within a region with relevant crime activity. The } \\
\text { exact meaning of "relevant" will be clear when we present the mechanism we } \\
\text { designed for hotspot detection. }\end{array}$ \\
\hline Hotspots Groups & $\begin{array}{l}\text { They are groups of anchor points or sites that share similar temporal behavior. } \\
\text { This concept enables the analysis of hotspots over time, making possible to } \\
\text { identify hotspots with similar behavior. }\end{array}$ \\
\hline Intensity & $\begin{array}{l}\text { It is the number of crimes in each anchor point or site. The intensity can be } \\
\text { measured in a given time unit or aggregated in a time unit interval. }\end{array}$ \\
\hline Probability & It is a measure of how likely a crime event is in each anchor point or site. \\
\hline Crime Pattern & $\begin{array}{l}\text { It accounts for the prevalence of a group of crime types in a region or sites. } \\
\text { In other words, if we say that the crime pattern in a set of sites is robbery, } \\
\text { car theft, and commercial establishment attack, we mean that the three crime } \\
\text { types are the most prevalent ones in those sites (GARCIA-ZANABRIA et al., } \\
\text { 2019). It can also account for the temporal behavior of a crime in a given site or } \\
\text { anchor point (GARCIA-ZANABRIA et al., 2020b). For instance, a place where } \\
\text { crimes are prevalent for a period of time, and then vanish gives rise to a crime } \\
\text { pattern (GARCIA-ZANABRIA et al., 2020a). }\end{array}$ \\
\hline
\end{tabular}

Source: Elaborated by the author. 


\subsubsection{Data Set 1 (2000 - 2006) - São Paulo}

The first data set considers only crime events related to passerby, vehicle, and commercial establishment, burglary, and larceny are provided, leaving out murder, homicide, drug-related felony, and sexual assault.

Each record contains the identification number of the census unit (site) where the crime happened, the type of crime, and the date and time of the crime event. The data set contains crime records from 2000 to 2006. At the beginning of our studies, we noticed that the information for 2005 and 2006 was not consistent with previous years, and a sanity check needed to be performed by the experts. Since the sanity check turned out more complex than expected, we opt to include only information from 2000 to 2004 in our studies, in a total of 1,574,920 records.

Crime types range in 121 categories, and the $10 \%$ most frequent crime types correspond to about $80 \%$ of the total crimes. Figure 9 shows the histogram of the $10 \%$ most prevalent crime types, labeling the three most frequent ones, passerby robbery, auto theft, and passerby larceny. To facilitate the analysis, experts split the original data into three independent categories, vehicle robbery (includes cars, motorcycles, and truck) with 295,081 instances, larceny in general, with 587,885 instances, and the third category with all the other types of robbery and burglary, with 691,954 instances.

Although the number of crime types is quite large (121 categories), the crimes that

Figure 9 - Histogram of the $10 \%$ most prevalent crime types, labeling the three most frequent ones, passerby robbery, auto theft, and passerby larceny

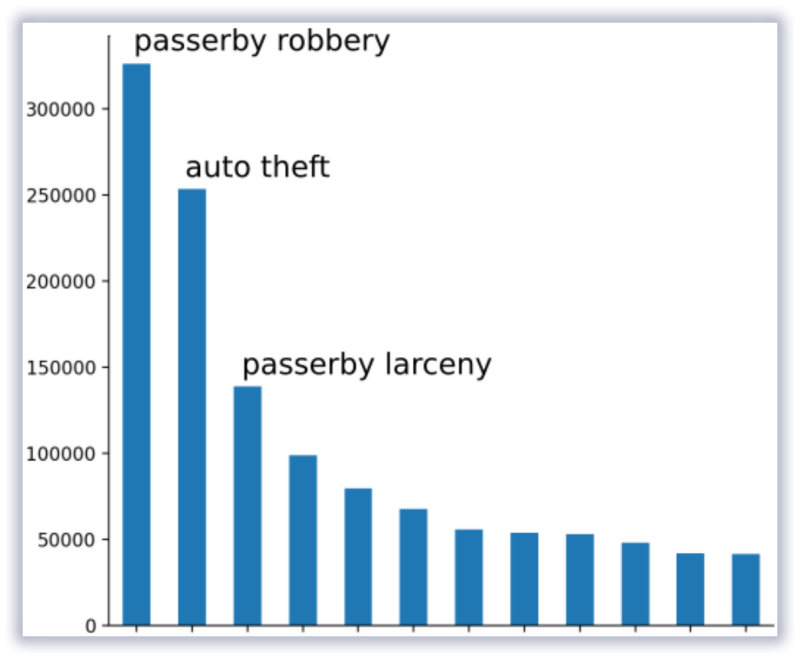

Source: Garcia-Zanabria et al. (2019). domain experts are interested in are not that large, ranging from three to five (passerby, auto, commercial establishment, document, and residential robbery). Other crime types are sparse enough to be analyzed individually and do not require sophisticated visualization tools to interpret them. Moreover, some crimes can be grouped into categories.

\subsubsection{Data Set 2 (2006 - 2017) - São Paulo}

The second data set consists of information on about 1,650,000 incidents of criminal activities recorded from 2006 to 2017. We have worked with three crime types: passerby, commercial establishment, and vehicle robbery. Each record contains the identification ID of the census unit where the crime happened, the date and time of the crime, type, and the geocode 
(latitude and longitude) information. Moreover, each instance contains a quality label of the geocoded process.

The geocoded quality labels range in five categories, and more than $91 \%$ of the records were successfully geocoded. Figure 10 shows a histogram of the number of criminal records over the years, and the quality label of the data, Ouro, Prata, Bronze, Pirita, and Chumbo. Ouro are records with two validation processes, Prata are records with one validation process, Bronze are records that could not be validated, but there is no evidence of being wrong. These three first groups of records present both coordinates (latitude and longitude) and the code of sites. Pirita records present some geographic reference, but there is some evidence that they are wrong. Finally, Figure 10 - Distribution of crime data from São Paulo over the years, and the quality label of the data, Ouro, Prata, Bronze, Pirita, and Chumbo

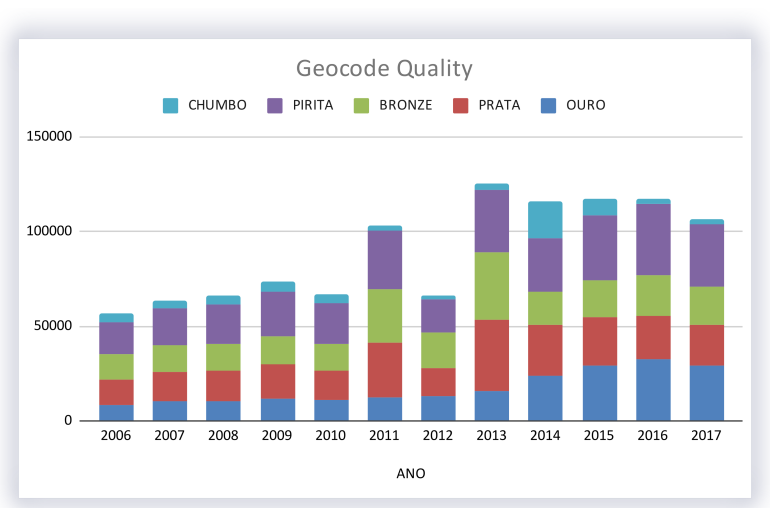

Source: Elaborated by the author.

Chumbo records have no geographic information. In this work, we have used Ouro, Prata, and Bronze.

\subsubsection{Data Set 3 (2014 - 2019) - São Carlos}

One of our case studies involves the crime activity of a medium-sized city. The third data set involves crime activities from 2014 to 2019 in São Carlos - São Paulo Brazil. Each record contains the identification ID, the date and time of the crime, crime type, and the geocode (latitude and longitude) information.

Figure 11 shows a histogram of the number of criminal records by crime type. It can be noticed that commercial establishment, passerby, and vehicle robbery are the most prevalent ones.
Figure 11 - Histogram of the number of criminal records by crime type.

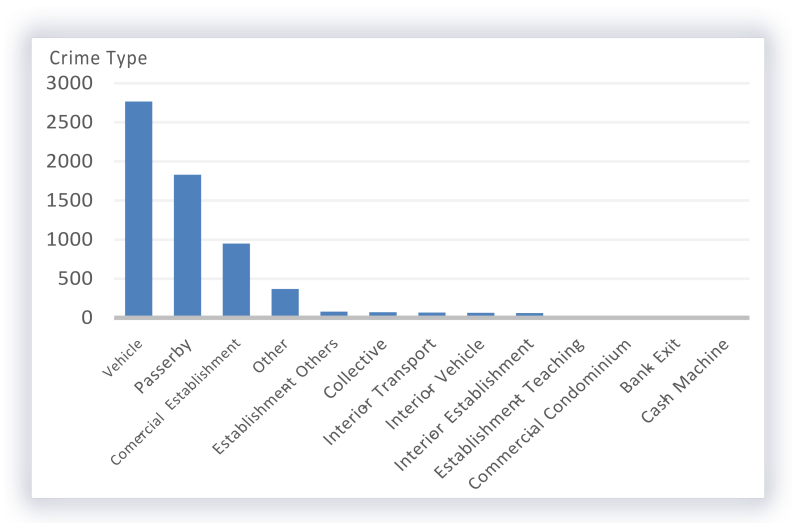

Source: Elaborated by the author. 


\subsection{Final Considerations}

In this chapter, we presented the terminology that will be used in the rest of the text. Moreover, we presented three data sets used in the case studies of our approaches. The first two involve criminal events of São Paulo city from 18 years. On the other hand, the third data set contains the events from 2014 to 2019 of São Carlos City. In our approaches presented in the next chapters, we modeled these data sets based on experts' requests and various analytical tasks. 

CHAPTER

4

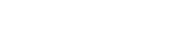

\section{CRIMANALYZER: UNDERSTANDING CRIME PATTERNS IN SÃO PAULO}

\subsection{Initial Considerations}

Most existing tools developed for crime mapping focused on the detection of hotspots, that is, areas with a high number of criminal incidents (ECK et al., 2005). Hotspot analysis is a ubiquitous technique used to visualize where the crime tends to be the highest. Although sophisticated mechanisms have been proposed to detect hotspots (EFTELIOGLU; SHEKHAR; TANG, 2020), the search for a high prevalence of crimes ends up neglecting sites where certain types of crimes are frequent but not sufficiently intense to be considered statistically significant (WANG et al., 2013a). Moreover, most techniques enable only rudimentary mechanisms to analyze an important component of unlawful activities, the temporal evolution of crimes and their patterns. In fact, visualization resources for temporal analysis available in the majority of crime mapping systems are very restrictive, impairing users from performing elaborated queries and data exploration (ANDRESEN, 2017).

Another important aspect to be considered in this context is the specificities of urban areas under analysis. Big cities such as São Paulo, bears one of the highest crime rates in the world, at least one order of magnitude higher than cities such as New York and San Francisco, making glyph-based crime mapping solutions such as LexisNexis ${ }^{1}$, NYC Crime Map ${ }^{2}$, and CrimeMapping ${ }^{3}$ unsuitable for analyzing crimes in São Paulo. Nevertheless, the pattern of crimes (in this chapter, crime patterns account for the prevalence of a group of crime types in a region or sites) change considerably around São Paulo, even between regions that are geographically close to each other, demanding analytical solutions tailored to reveal local hotspots and corresponding

communitycrimemap.com

maps.nyc.gov/crime/

crimemapping.com 
crime patterns. Such local solutions should also be able to uncover the dynamic of hotspots over time. Those capabilities are not currently available in most crime mapping tools.

In this chapter, we present CrimAnalyzer, a new visual analytic tool customized to support the analysis of crime activities in urban areas with specific characteristics, that is, high criminality rates with great variability in the pattern of crimes, even in geographically close regions. CrimAnalyzer enables a number of linked views tailored to reveal patterns of crimes and their evolution over time, assisting domain experts in their decision-making process and providing guidelines not only for repressive but above all preventive actions, strengthening the planning and implementation of institutional actions, especially from the police.

In collaboration with a team of domain experts, we have designed visual analytic functionalities that allow users to select and analyze regions of interest in terms of their hotspots, crime patterns, and temporal dynamics. Moreover, the proposed system enables resources for users to dig deeper in particular sites to understand their prevalent crimes and their behavior over time. Furthermore, CrimAnalyzer implements a technique based on Non-Negative Matrix Factorization (LEE; SEUNG, 2001) to identify hotspots based not only on the number of crimes but also on the rate they occur.

\subsection{Challenges and Analytical Tasks}

\subsubsection{Problem Analysis}

We had several rounds of meetings and interviews with the experts to identify the main challenges involved in the analysis of crime data. After several interactions, we came up with the following issues:

- Analyzing the characteristics and dynamics of crimes in particular regions of the city. From their experience and interaction with officers from the police department, the experts conjecture that the type and dynamic of crimes have been changing over the years, mainly in particular regions of the city. Moreover, the type of crimes can change even in regions located close to each other depending on the urban characteristics of each region. The main difficulty to perform this analysis without a visual analytics tool is to properly query the data set, which can be a time-consuming and exhausting task. Moreover, highly prevalent crimes overshadow the presence of less frequent ones, which might also be of interest, demanding specific tools to enable a proper analysis. Given the difficulties, the experts have been performing their analysis focusing on just one or two types of crime, considering the city as a whole or analyzing large areas that serve as administrative units within the city. Such broad analysis hampers the verification of hypothesis and conjectures that have a local nature.

- Identifying crime hotspots within a particular region. The identification of crime hotspots is among the most important tasks when analyzing crimes and their dynamics. Hotspots 
are usually identified as locations that have a greater than the average number of criminal records (ECK et al., 2005). However, crime sites that are not so prevalent in terms of the number of criminal events, but bear crime activities that deserve special analysis, tend not to be detected when a "Intensity" approach is employed to identify crime hotspots. Due to the lack of more sophisticated mechanisms, the number of criminal records has been the main mechanism employed by the experts in their identification of hotspots. Because of this, it was necessary to propose a new technique for hotspot detection that meets the described restrictions. This requirement was emphasized by the domain experts.

- Understanding and comparing crime patterns. Domain experts believe that sites and hotspots within the same region can present different crime patterns. An issue in this context is to know whether the pattern of crime varies from a site (or hotspot) to another in the same region. In affirmative case, experts would like to understand how crime types are distributed and how they evolve along time. The experts were looking for a solution that would intuitively allow them to make such comparisons.

Challenges above point to a visual analytic solution endowed with functionalities to easily select regions of interest while enabling resources to assist the analysis of crime location, patterns, and temporal evolution. We followed a design process that involved the experts in most stages of the development (MUNZNER, 2009), redesigning procedures, components, and functionalities according to experts feedback and demands.

\subsubsection{Analytical Tasks}

After identifying the main challenges faced by the experts and understanding how the data was structured, we conducted a new series of interviews to raise questions to be investigated. It has become clear that the experts are interested in understanding the dynamics of crimes over the city by analyzing the variation of crime patterns over space and time. From the iterative processes with the experts, we compiled the following list of analytical tasks:

- Interactive selections (T1): How can spatial regions of interest be interactively selected? Is it possible to make the interactive selection of regions flexible enough to pick from single spots to whole neighborhoods and particular avenues?

- Crime patterns over the city (T2): Which are the crime patterns in particular regions and sites? How do criminal patterns change from the center to residential areas and outskirts? What about the patterns along the main avenues, streets, and highways?

- Dynamic of crimes over time (T3): How have crime types evolved, over time, in particular regions of the city?. More specifically, have crime patterns changed in particular regions over the years? Are crime types seasonal?

- Crime patterns and hotspots over space (T4): Which are the hotspots in a given region? Which are their crime patterns? How different (if the difference exists) are the crime patterns 
in distinct hotspots within the same region?

- Crime patterns and hotspots over time (T5): Have crime hotspots changed over time in a given region? Have crime patterns changed over time in a given hotspot?

As mentioned before, the lack of interactive mechanisms to select regions of interest combined with general-purpose analysis and visualization techniques have prevented domain experts from freely exploring the data to verify hypotheses and conjectures. The first step to enable more powerful analytic resources is the design of a proper interactive selection tool, being this the goal of $\mathrm{T} 1$.

It has also become clear during the interviews that it is important to drill down from high-level summaries to individual analysis of sites and hotspots. Analyzing data in different scales is also essential to understand how patterns vary across space and time. For example, the pattern of crimes and hotspots can change throughout months and over different days of the week. This fact is related to T3, and requires particular data aggregation and filtering to be properly addressed.

Analytical tasks T2 and T3 are related to the problem of understanding the different patterns of crimes around the city and their evolution over time, as discussed in Section 4.2.1, while tasks T4 and T5 are associated to the problem of analyzing hotspots, also discussed in Section 4.2.1. To be properly addressed, those tasks demand specific mechanisms to detect hotspots and also visual resources to explore and understand them.

Among our goals is the integration of interactive selection methods and dedicated visual analysis tools towards allowing domain experts to accomplish both confirmatory and exploratory analysis. Moreover, some domain experts are not trained in computer science, thus, the system should be as simple and intuitive as possible. However, simplicity and expressiveness must be balanced to render the system capable of supporting spatio-temporal analysis at different scales, while being able to uncover non-trivial hotspots and crime patterns.

\subsubsection{The CrimAnalyzer System}

Based on the requirements and analytical tasks outlined above, we have developed CrimAnalyzer, a system for exploring spatio-temporal crime data in specific locations. CrimAnalyzer enables simple, yet compelling, visual resources to query, filter, and visualize crime data. The visual resources are supported by a mathematical and computational machinery tailored to extract and polish information so as to visually present it in an intuitive and meaningful way. The modules and system architecture are illustrated in Figure 12. Users visually query the data set by interacting with a map and selecting a region of interest as well as by interacting with the different linked views that make up the system. 
Figure 12 - Pipeline overview of the CrimAnalyzer System.

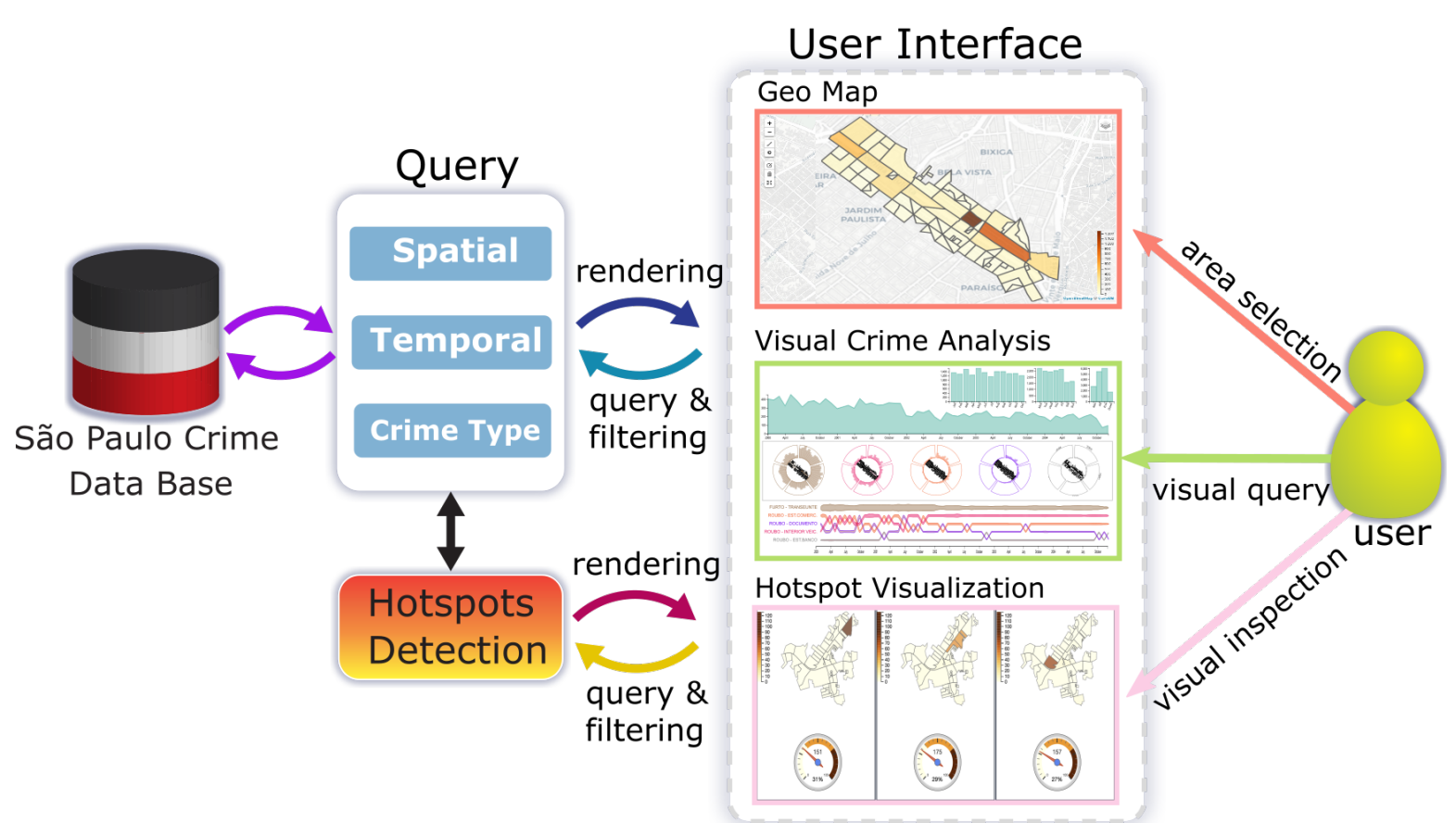

Source: Garcia-Zanabria et al. (2019).

\subsection{Hotspot Identification Model}

Hotspot identification is one of the most important tasks for crime analysis. In our context, hotspots have a more general connotation than in previous works, corresponding to sites where criminal activity is high but also to locations where the number of crimes is not large, but frequent enough to deserve a detailed analysis. For example, a region can contain sites where the number of crimes is much larger than in any other sites, which clearly correspond to important hotspots. However, the region can also contain a particular site where crimes are frequent, but happening in much smaller magnitude if compared against the prominent ones. Moreover, the region can also contain sites where crimes are not frequent at all, but present spikes in particular time frames. In our context, we consider the three different phenomena as hotspots, seeking to identify sites where crimes are frequent and in large number, sites where crimes are frequent but not in large number, and sites where crimes are not frequent, but happen in large numbers in particular time frames. Different crime behaviors will be further discussed and illustrated in Section 4.3.2.

Analysis of individual sites. There are many alternatives to identify hotspots, as discussed in Section 2.3. Although varying in terms of complexity, existing techniques typically rely on the comparison of statistical information. Hotspots can be identified as particular points or as area units, depending on how the data is organized, delegating to the visualization the task of properly revealing the hotspots. The problem with this approach is that crimes happening in small magnitude or in isolated time frames tend not to be statistically significant, hardly being pointed out as hotspots. 
Another issue is that several sites might be identified as hotspots, but their temporal relation remains unrelated. For example, sites can be timely correlated, meaning that crimes are committed in those sites in the same time slices. It makes sense to group timely correlated sites in a single hotspot, but computing hotspots individually and group them according to temporal matches is not easy and involves the use of thresholds to decide which sites should be grouped.

Analysis of groups of sites. Instead of analyzing sites individually, one can resort to techniques that directly identify groups of sites as hotspots. A straightforward alternative is to extract features from the sites and apply a clustering scheme to group similar sites in hotspots (see Section 2.3). However, the problem of extracting meaningful features that characterize sites spatially and temporally is quite involved, mainly due to the sparsity of the crime data. In fact, in the course of our development, we tried different alternatives to define spatio-temporal crime feature vectors, ranging from simple cumulative time windows to more sophisticated methods based on graph wavelet coefficients (VALDIVIA et al., 2015), but we could not obtain results that complied with our requirements.

To get around the difficulties pointed above, we opted for an approach based on NonNegative Matrix Factorization (LEE; SEUNG, 2001), which worked pretty well in our context, identifying hotspots according to our requirements.

\subsubsection{Non-Negative Matrix Factorization}

Before presenting the details on how we have adapted Non-Negative Matrix Factorization (NMF) to operate in our context, lets shortly review the main concepts and ideas involved in an NMF analysis. An $m \times n$ matrix $X$ is said non-negative if all entries in $X$ are greater or equal to zero $(X \geq 0)$. The goal of NMF is to decompose $X$ as a product $W \cdot H$, where $W$ and $H$ are non-negative matrices with dimensions $m \times k$ and $k \times n$, respectively (the roles of $m, n$, and $k$ will be clear in Section 4.3.2). In mathematical terms, the problem can be stated as follows:

$$
\arg \min _{W, H}\|X-W H\|^{2} \quad \text { subject to } W, H \geq 0
$$

A solution for the minimization problem (Equation 4.1) provides a set of basis vector $w_{i}$, corresponding to the columns of $W$, and a set of coefficients $h_{j}$, corresponding to the columns of $H$, such that each column $x_{j}$ of $X$ is written as the linear combination $x_{j}=\sum_{i} h_{i j} w_{i}$, (or $x_{j}=W h_{j}$ ). In other words, for each line in $X$ we have a corresponding column in $H$ whose entries are coefficients associated to the columns (basis vectors) of $W$. The matrix representation below (Equation 4.2) illustrates the relation between columns of $X$ and $H$ as well as coefficients in $H$ and basis vector in $W$.

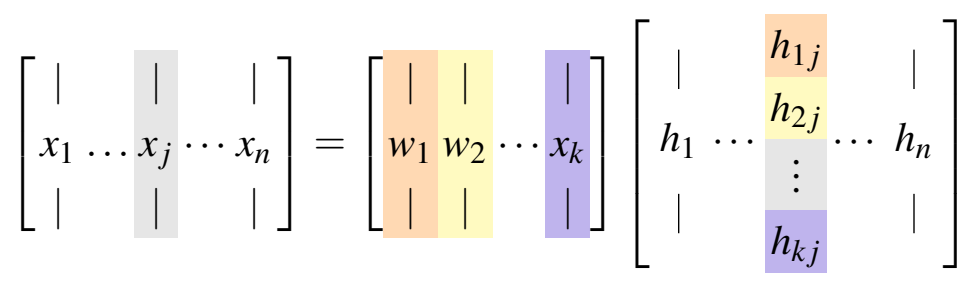


There are two important aspects in an NMF decomposition that will be largely exploited in the context of hotspot detection, namely, low rank approximation and sparsity. Low rank approximation accounts for the fact that the basis matrix $W$ usually has much lower rank than the original matrix $X$, meaning that (the columns of) $X$ is represented using just a few basis vectors. As detailed in the next subsection, we rely on low rank approximation to define the number of hotspots, that is, by setting the rank of $W$ we also set the number of hotspots. Sparsity means the basis and coefficient matrices contain many entries equal (or close) to zero, which naturally enforces only relevant information from $X$ to be kept in $W$ and $H$. This fact is important to identify particular sites within a hotspot and the time slices where each hotspot shows up.

Figure 13 - a) Region of interest. b) Data matrix containing crime information from the regions in a). Rows correspond to sites while columns are time slices. The darker the color, the closer to zero the number of crimes is. c) Rank 3 NMF decomposition from $X$. d) Rank 5 NMF from $X$.

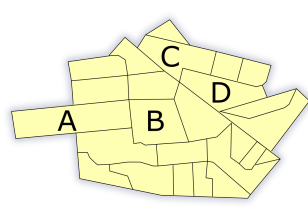

(a) Region of Interest

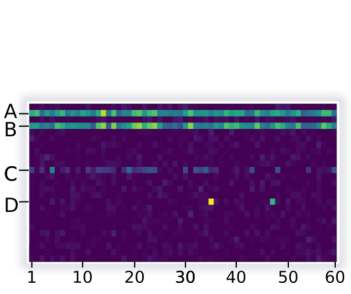

(b) Data matrix $X$

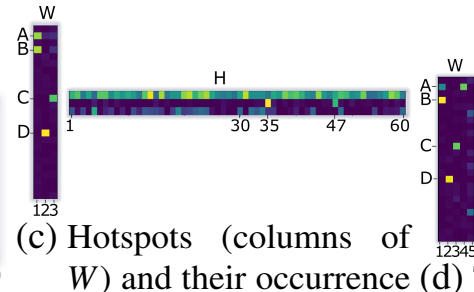

(rows of $\mathrm{H}$ )

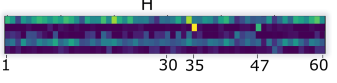

(d) The same as c) but with rank 5

Source: Garcia-Zanabria et al. (2019).

\subsubsection{Identifying Hotspots with NMF}

We rely on NMF to identify hotspots, their rate of occurrence and "intensity". The matrix $X$ to be decomposed as the product $W \cdot H$ comprises crime information in a particular region of interest. Specifically, each row in $X$ corresponds to a site of the region and each column to a time slice. In order to facilitate discussion, we present the proposed approach using a synthetic example. Figure 13 shows a region made up of 25 sites and we generated synthetic crime data in 60 time slices, representing months over five years. For sites denoted as $A$ and $B$, we draw 60 samples from a normal distribution with mean 8 and variance 4 , ensuring that $A$ and $B$ are correlated, that is, when the number of crimes in $A$ is large the same happens with $B$ (the number of crimes in $B$ is generated by perturbing the values of $A$ using a uniform random distribution with values between -3 and 3 ). This construction is simulating two regions with high prevalence of crimes over time. Crimes in the site denoted as $\mathrm{C}$ in Figure 13 follows a normal distribution with mean 1 and variance 4, corresponding to a location where crimes are not large in number, but happening quite frequently. Finally, for site $D$ we draw 60 samples from a normal distribution with mean 0 and variance 0.25 , except for time slices 35 and 47 , where we set the number of crimes equal to 15 and 10 respectively, simulating a site where crimes are no frequent, but happen in large numbers in particular time slices. For all the other sites we associated 60 samples drawn 
from a normal distribution with mean 0 and variance 0.25 . Values for all sites are rounded to the closest integer and negative values set to zero. Figure 13b illustrates the matrix $X$ built from the synthetic data described above. Notice that the simulated crime dynamics is clearly seen in $X$.

Given an $m \times n$ matrix $X \geq 0$, an NMF decomposition of $X$ results in matrices $W \geq 0$ and $H \geq 0$. In practice, the rank of $W$ is significantly less than both $m$ and $n$, i.e., $k=\operatorname{rank}(W) \ll m, n$. In our context, the columns of $W$ correspond to hotspots while entries in the rows of $H$ indicate the "intensity" of the hotspot in each time slice. Figure $13 \mathrm{c}$ illustrates matrices $W$ and $H$ obtained from matrix $X$ in Figure 13b using a NMF decomposition with rank $k=3$. Notice that the entries in first (left most) column of $W$ have values close to zero almost everywhere, except in the entries corresponding to the sites $\mathrm{A}$ and $\mathrm{B}$. Therefore, the hotspot derived from the first column of $W$ highlights sites $A$ and $B$ as the relevant ones. The high prevalence of crimes on those regions can clearly be seen from the first (top) row of matrix $H$, which has most of its entries with non-zero values. The second column of $W$ is mostly null, except in the entry corresponding to site $D$, where crimes are not frequent but happen with high intensity in particular time slices. Notice that the second row of $H$ has basically two entries different from zero, corresponding exactly to the time slices 35 and 47, when the site D faces a large number of crimes. Finally, the last column of $W$ gives rise to a hotspot that highlights site $C$, where crimes are frequent, but in smaller magnitude when compared to $A$ and $B$. The incidence and intensity of crimes in $C$ are clearly seen in the third (bottom) row of $H$.

One can argue that the results presented in Figure 13c worked so well because we wisely set the rank of $W$ equal $k=3$ and that in practice it is difficult to find a proper value for the rank. To answer this question, Figure 13d shows the result of factorizing matrix $X$ setting the rank of $W$ equal $k=5$. Notice that the main difference between the rank $k=3$ and $\operatorname{rank} k=5$ factorizations is that the first column of $W$ in Figure $13 \mathrm{c}$ was split into two columns in the rank $k=5$ factorization, giving rise to columns 1 and 3 of $W$ in Figure 13d. Nevertheless, the third column still indicates the correlation between $A$ and $B$, which thus is not completely missed due to the presence of column 1. The right most column of $W$ in Figure $13 \mathrm{~d}$ is mostly noise and it represents sites with a little criminal activity, what is attested by the bottom row of $H$ in Figure 13d, which is almost null. Therefore, increasing $k$ tends to split meaningful hotspots while creating some noisy, not so important ones, which can easily be identified from almost zero rows in $H$.

Improving the identification of hotspots. Most entries in matrix $H$ are close to but are not zero, demanding a threshold to decide whether or not a hotspot takes place in a given time slice. Playing with thresholds is always inconvenient, mainly for non-experienced users. In order to avoid the use of thresholds, we binarize the matrix $H$ using the Otsu's algorithm (OTSU, 1979), considering that a hotspot appears in a given time slice if its corresponding entry in the binarized version of matrix $H$ is 1 . 
The synthetic example discussed above shows that hotspots generated from NMF attend the requirements of our problem, justifying our choice of NMF as mathematical model for tackling the problem. Among the different versions of NMF, we opt to the sparse non-negative matrix factorization proposed by Kim and Park (KIM; PARK, 2007), which allows for enforcing sparsity in both $W$ and $H$ simultaneously.

We conclude this section saying that, as far as we know, this is the first work to employ NMF as a mechanism to detect hotspots in the context of Crime Mapping.

Comparison with spatial statistics The Getis-Ord $G_{i}^{*}$ statistics (GETIS; ORD, 1992; ORD; GETIS, 1995) is a well-known hotspot detection method available in the toolbox Local Indicator of Spatial Association (LISA) (ANSELIN, 1995) $G_{i}^{*}$ operates by measuring the local spatial autocorrelation variation over a region of interest. $G_{i}^{*}$ reports a $\mathrm{p}$-value and a z-score for each location in the region of interest, marking as hotspots those with statistically significant (low p-values) large z-scores.

Figure 14 - (a) Division of São Paulo into 300 groups and (b) SSI distribution in those regions. NMF and $G_{i}^{*}$ detect the same hotspots in most of the cases.

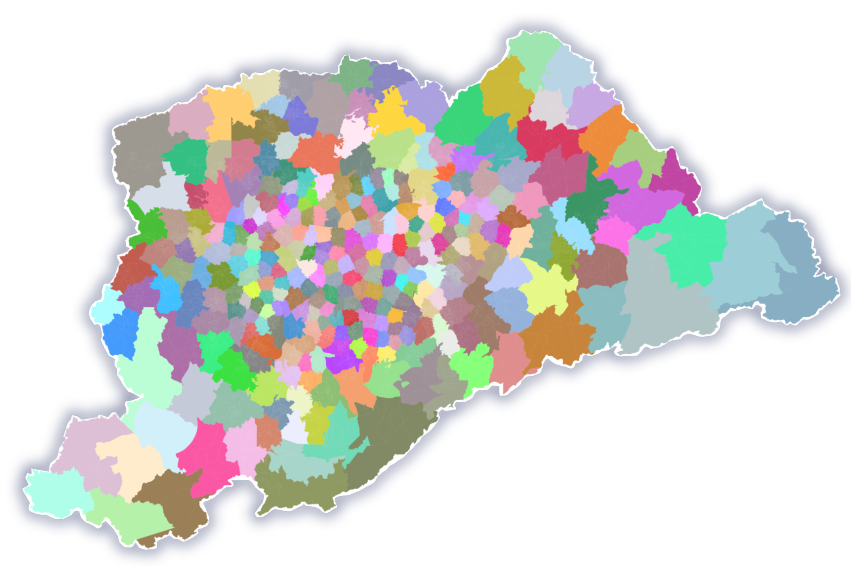

(a) São Paulo clustering

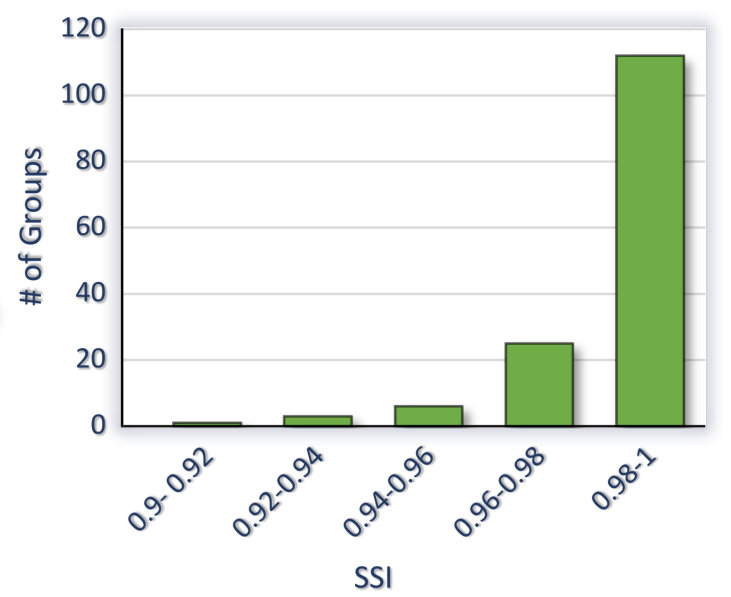

(b) SSI distribution for $k=3$

Source: Garcia-Zanabria et al. (2019).

In order to perform a quantitative comparison between NMF and $G_{i}^{*}$, we grouped the census units into 300 regions as shown in Figure 14a. The regions have been computed by applying K-means to the coordinates of the centroid of the census units. Since there are about 30,815 census units, setting the number of clusters equal to 300 tends to generate groups with about 100 units in the denser areas of the city. The Sokal-Sneath index (SSI), a well known binary data classification similarity measure (S; CHA, 2010), is employed to compare the hotspots resulting from NMF with $k=3$ (the default rank value in our system) against the ones obtained by $G_{i}^{*}$ with a $99 \%$ confidence level (we relied on the $G_{i}^{*}$ implementation available in 
the PySAL Python library (REY; ANSELIN, 2007)). Specifically, we assign each site to one of the four categories (labels):

- P: if the site is a hotspot for both NMF and $G_{i}^{*}$ (positive match);

- F: if the site is a hotspot detected by NMF, but not by $G_{i}^{*}$;

- G: if the site is a hotspot detected by $G_{i}^{*}$, but not by NMF;

- $\mathrm{N}$ : if the site is not a hotspot for both methods (negative match).

The SSI similarity measure is then computed as:

$$
S S I=\frac{2|\mathrm{P}|+2|\mathrm{~N}|}{2|\mathrm{P}|+|\mathrm{F}|+|\mathrm{G}|+2|\mathrm{~N}|},
$$

where $|\cdot|$ denotes the cardinality. A $S S I=1$ means that the hotspots detected by both methods in a given region match exactly.

Figure 15 - Qualitative comparison between NMF and $G_{i}^{*}$. (a) Region where NMF detects more hotspots and (b) region where $G_{i}^{*}$ detects more hotspots. The darkest sites are the ones with high criminality.

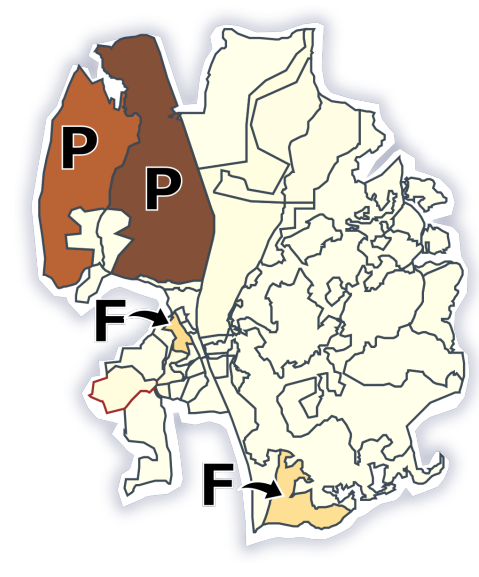

(a) $|\mathrm{F}|=2$ and $|\mathrm{G}|=0$

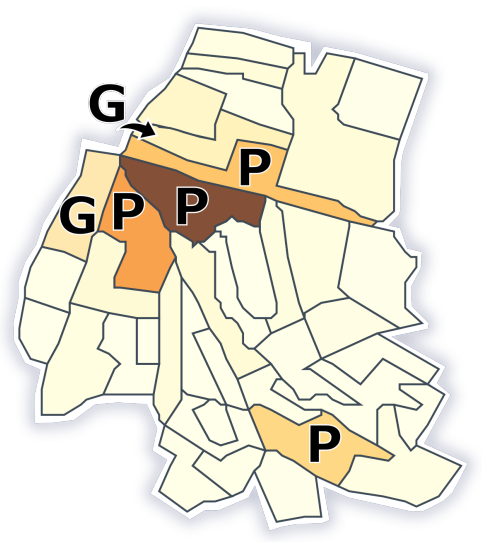

(b) $|\mathrm{F}|=0$ and $|\mathrm{G}|=2$

Source: Garcia-Zanabria et al. (2019).

Histogram depicted in Figure 14b gathers SSI values from all the 300 regions. Note that $S S I$ values are larger than 0.90 , most of them lying in the range $[0.98,1.00]$, showing the good match between NMF and $G_{i}^{*}$. In fact, most of the locations pointed out as hotspot by $G_{i}^{*}$ are also captured by NMF. However, in about 200 regions, NMF detected a few more hotspots than $G_{i}^{*}$.

Figure 15 illustrates typical situations where NMF and $G_{i}^{*}$ differ in a few unities. In Figure 15a, NMF and $G_{i}^{*}$ have both found the hotspots labeled as P (the labels in Figure 15 are according to the classification used by SSI, the darker the site is, the more dangerous it is), but NMF has captured two extra hotspots, labeled as F (unlabeled units belong to the category $N$ ). Notice that the color code indicates that the sites $F$ are indeed regions with a prevalence of 
criminality, although not captured by $G_{i}^{*}$ (the darkest sites are the ones with high criminality). In Figure 15b, in contrast, $G_{i}^{*}$ detects two more hotspots than NMF (G sites). Notice that the color code of the units marked as $G$ in Figure $15 b$ indicates that crimes in those regions are not so intense as in the $\mathrm{P}$ hotspots. The reason why $G_{i}^{*}$ points the $\mathrm{G}$ sites as hotspots is that those sites are neighbors of units where the number of crimes is high ("real" hotspots), so the kernel integration employed by $G_{i}^{*}$ ends up being contaminated by the neighbor sites where crimes are prevalent. In other words, the $G$ sites are pointed out as hotspots due to their proximity with $\mathrm{P}$ sites. Sites pointed as $\mathrm{F}$ in Figure $15 \mathrm{a}$ have not been captured by $G_{i}^{*}$ because they are isolated in the middle of units with no crimes. Therefore, besides not demanding a grid discretization, NMF tends to capture hotspots in a more consistent manner, being an attractive alternative to conventional statistical approaches.

The value $k$ (NMF rank) impacts the SSI measure. We have run the comparisons ranging $k=3, \ldots, 10$, getting an average $S S I$ greater than 0.98 for $k=3,4,5$, but slightly better for $k=3$. This result motivated us to set $k=3$ as the default value in CrimAnalyzer.

Table 3 - View properties and their analytical tasks (Sec. 4.2.2).

\begin{tabular}{cccccc} 
& T1 & T2 & T3 & T4 & T5 \\
\hline \hline Map View & $\checkmark$ & $\checkmark$ & & $\checkmark$ & \\
Hotspot View & $\checkmark$ & & & $\checkmark$ & $\checkmark$ \\
Cumulative Temporal View & $\checkmark$ & & $\checkmark$ & & $\checkmark$ \\
Global Temporal View & $\checkmark$ & & $\checkmark$ & & $\checkmark$ \\
Ranking Type View & $\checkmark$ & $\checkmark$ & $\checkmark$ & $\checkmark$ & $\checkmark$ \\
Radial Type View & $\checkmark$ & $\checkmark$ & $\checkmark$ & $\checkmark$ & $\checkmark$ \\
\hline \hline
\end{tabular}

Source: Garcia-Zanabria et al. (2019).

\subsection{Visual Design}

This section describes the visual components of CrimAnalyzer. Figure 16 illustrates the web-based system, which comprises a Control Menu (a) six interactive views (b-g), and a filter widget (h). Table 3 shows the properties of each view. For instance, Map View shows the space facet and Ranking Type View the temporal and crime type facets. The design of visual resources was driven by the analytical tasks described in Section 4.2.2. In Table 3, Columns under task's category indicate the relation of each view and the analytical tasks. For instance, Ranking Type View and Radial Type View account for all analytical tasks.

In the area of crime analysis, visualizations have always been used to display the data; however, improvements or new designs over existing visualizations are needed. For instance, the Ranking Type View is a novel alternative visualization in this context, which turns out to be effective to elucidate the dynamics of different types of crime over time in specific locations of the city. Although this visual metaphor is well known by the visualization community, it has never been used for crime analysis. 
In the following, we describe each visual components, starting with the Control Menu (see Figure 16).

Figure 16 - CrimAnalyzer system: the spatial and temporal interactive views enable the exploration of local regions while revealing their criminal patterns over time.

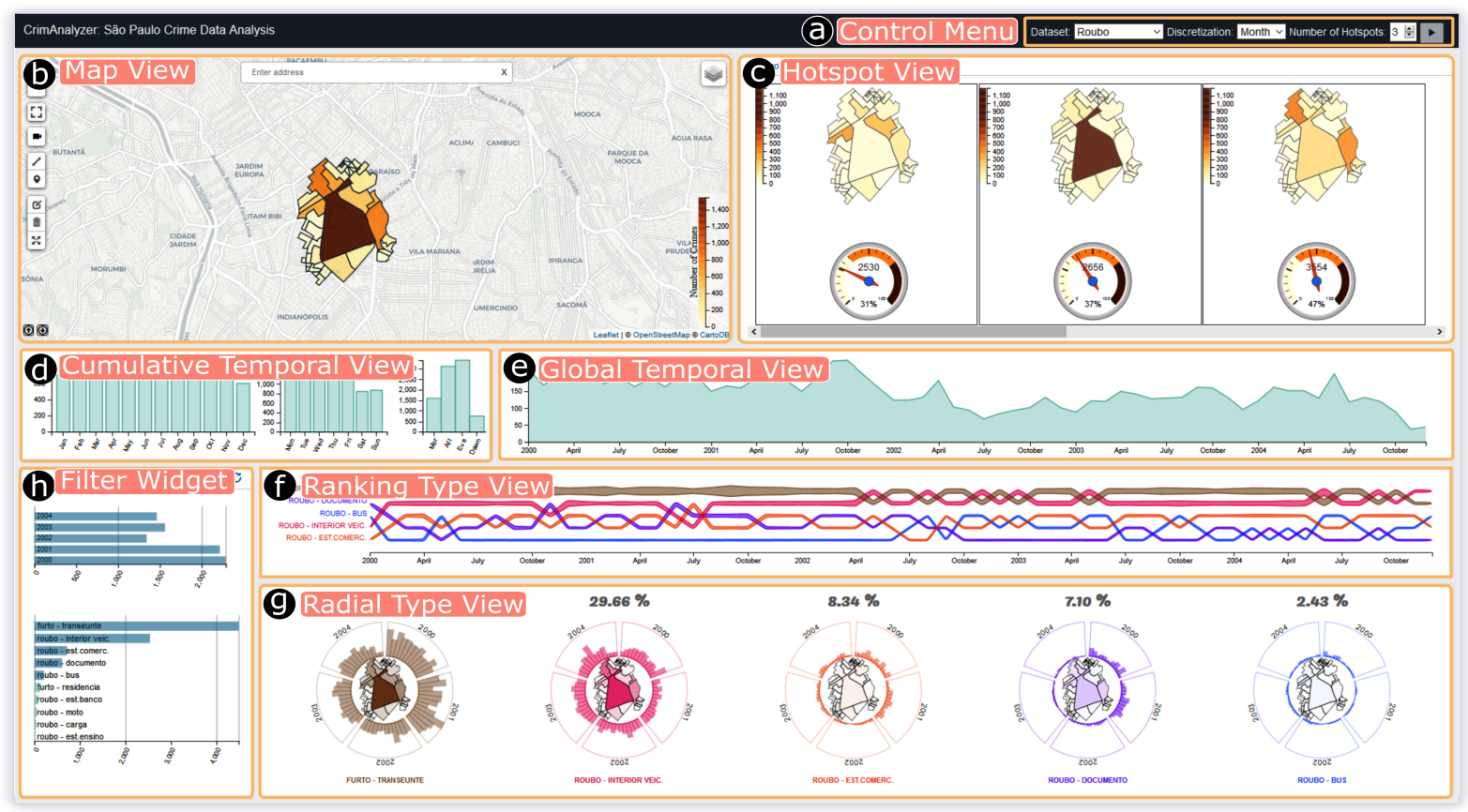

Source: Garcia-Zanabria et al. (2019).

\subsubsection{Control Menu}

The control menu has three options: dataset, time discretization (i.e., months or days) and the number of hotspots (rank of the NMF decomposition). As shown in Figure 16(a) we are using the dataset "Roubo" with monthly discretization and three hotspots $(k=3)$ in most of our analysis. The NMF decomposition is performed using the Nimfa Python library (ZITNIK; ZUPAN, 2012), which is able to evaluate and automatically choose the rank value $k$. However, such an automated process is computationally costly, limiting its use in an interactive visual analytics application such as CrimAnalyzer. Therefore, we have opted to allow the user manually set $k$.

\subsubsection{Map View}

This is the first component used to start the analytical process, where the user can define the region of interest. This view is comprised of a geographical map and a choropleth map to encode the number of crimes committed at each site in the region. Also, users can zoom and pan the map. 
Region selection: Users can define a region of interest by 1) clicking on the map (to select a site), 2) drawing a polyline (to select avenues or streets for example), 3) drawing a polygon (to select a whole neighborhood), or 4) provide the address of a location. Drawings can be expanded to include other sites in the neighborhood. Finally, CrimAnalyzer defines the region by computing the sites that intersect the drawing. In Figure 16(b) we can see how a region is represented.

Site selection: During the exploration, when a region has already been defined, this view might be used for spatial filtering (e.g., to focus on a particular site). This operation is performed by clicking a site, which is highlighted by mapping a texture to the corresponding area.

Filtering: When other views make spatial filtering (i.e., selecting a site), the corresponding site is highlighted by changing its texture. When a time or type filter is activated by other views, our choropleth map is recalculated using the filtered data.

\subsubsection{Hotspots View}

An important component of our approach is the hotspots identification. In Section 4.3.2, we explained how Non-Negative Matrix Factorization has been used to reveal hotspots. In this view, we use multiple maps to represent the spatial distribution of each hotspot. Users can specify the number of hotspots in the Control Menu. Below each hotspot (see Figure 16(c)), there is a gauge widget that depicts the number of crimes in the hotspot (the top number in the gauge), the temporal rate of occurrence of the hotspot (the bottom percentage in the gauge), and how relevant is that hotspot in the whole set of crimes (the gauge pointer). The importance of the hotspot is computed by a function $f:[0,1] \times[0,1] \rightarrow[0,1]$ that assign a value to each pair (rate_of_crimes, frequency_of_crimes), where rate_of_crimes denotes the number of crimes in the hotspot divided by the total of crimes and frequency_of_crimes is the temporal number of occurrences of the hotspot (computed for the binarized matrix $H$ ) divided by total number of time slices. In fact, $f$ is simply a bilinear interpolation in the unit square where $f(0,0)=0, f(0,1)=0.5, f(1,0)=0.7, f(1,1)=1$. With this distribution of values, we give more relevance to hotspots where the number of crimes is larger.

Selection: A hotspot selection filters the crimes in space and type. All the other views are recomputed to match the selected hotspot.

Filtering: Filtering the crimes using other views (i.e., space, time, or type) does not affect this view. If we want to recompute hotspots based on filtered data, for example, a particular crime type, we have to click the "Hotspots" button after performing the data filtering. 


\subsubsection{Global Temporal View}

This view gives an overview of the number of crimes committed over the whole time period, relying on a line chart with a filled area between the data value and the base zero line (see Figure 16(e)).

Time selection: In this view, we can constraint the analysis at a particular time interval, which can be defined by brushing a rectangle on the Global Temporal View. Only continuous time period can be selected. Next view will allow us to select multiple time intervals. All views (except the hotspot that needs to be recomputed) are affected and automatically adjusted accordingly to the time selection.

\subsubsection{Cumulative Temporal View}

This view uses a bar chart to present the number of crimes accumulated by month, day, and period of the day (see Figure 16(d)). In this view, we can see some patterns from non-continuous time intervals. This is also very useful to compare weekends or weekdays.

Filtering: When other views are used to filter the dataset, the filtered data is also overlaid on the global Cumulative Temporal View, thus enabling a comparative analysis (see Figure 20).

\subsubsection{Ranking Type View}

This view depicts three relevant pieces of information in a single metaphor: crime type evolution, crime type ranking, and number of crimes in each time slice. As shown in Figure 16(f), each crime type is represented by a polyline. The vertical position, on each time step, encodes the relevance compared to others. Moreover, the line width is proportional to the number of crimes belonging to it.

Filtering: When a filter is activated in other views, the ranking view is recomputed using the filtered data.

\subsubsection{Radial Type View}

In this view, we are using multiple bar charts with a radial layout (all charts share the same scale). Each chart represents a different crime type, for instance, in Figure 16(g) we have five crime types. In addition, the number on top of each chart shows the percentage for each crime type. Each chart is divided into sectors, where each sector is comprised of 12 bars depicting the months each year.

Crime Type selection: Clicking a chart filters the data to a specific crime type. In this way, users can focus their analysis on the most crime-prevalent types. Selected crime types are represented by a dashed borderline. 
Time selection: We provide interactivity features on each chart to enable comparison among the same month on different years and same month across different crime types.

Filtering: When the dataset is filtered, each chart is recomputed to represented the filtered data.

\subsubsection{Filter Widget}

This widget is comprised of a time and crime type histogram. For instance, Figure 16(h) summarizes our data in five years (2000 - 2004) and five crime types. Moreover, we use this histograms to filter our data. Clicking on a bar, we can remove a year or a crime type. This filtering affects the whole interface.

Although most of the presented visual resources are not novel individually, many of them (such as hotspot view, ranking type view, and radial type view) are non-trivial in the context of crime mapping. Even more important, the combination of all of them allows multiple analysis simultaneously, revealing interesting crime patterns, as shown in the next section.

\subsection{Implementation Details}

CrimAnalyzer is a web-based application implemented under the Django ${ }^{4}$ framework. The core of the system comprises hotspot calculation and visualization modules. For the hotspot calculation, we used Nimfa: A Python Library for Non-negative Matrix Factorization ${ }^{5}$. The visualization resources have been developed using JavaScript libraries: Leaflet ${ }^{6}$, to represent the criminality in a geo-map and D3.js ${ }^{7}$ to represent line and bar charts (including Ranking type view). In addition, extra components was used to manipulate filters (filter widget) by using Crossfilter ${ }^{8}$ and Dc.js ${ }^{9}$ libraries.

\subsection{Case Studies}

This section presents three case studies that show the effectiveness of CrimAnalyzer in addressing the analytical tasks presented in Section 4.2.2. The first case study addresses analytical tasks T1, T2, and T3, while the second focusses on hotspots analysis and it is related to T4 and T5. The third case study is aimed to make a parallel between criminal activity in São Paulo and some crime related phenomena reported in the literature (related to T3). In all case studies, except if explicitly stated, we used the robbery and burglary chunk of the dataset as described in Section 3.3.1, with a monthly discretization.

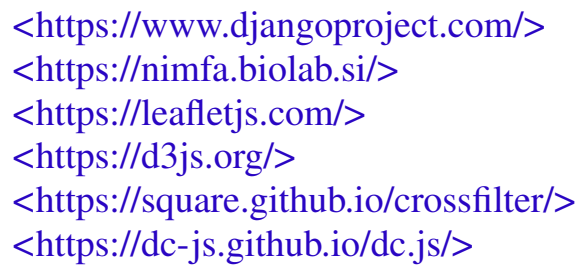


Figure 17 - Summary of criminal activities and corresponding patterns in four different regions of São Paulo. Crime patterns might change substantially among the regions and also along the time (five years).

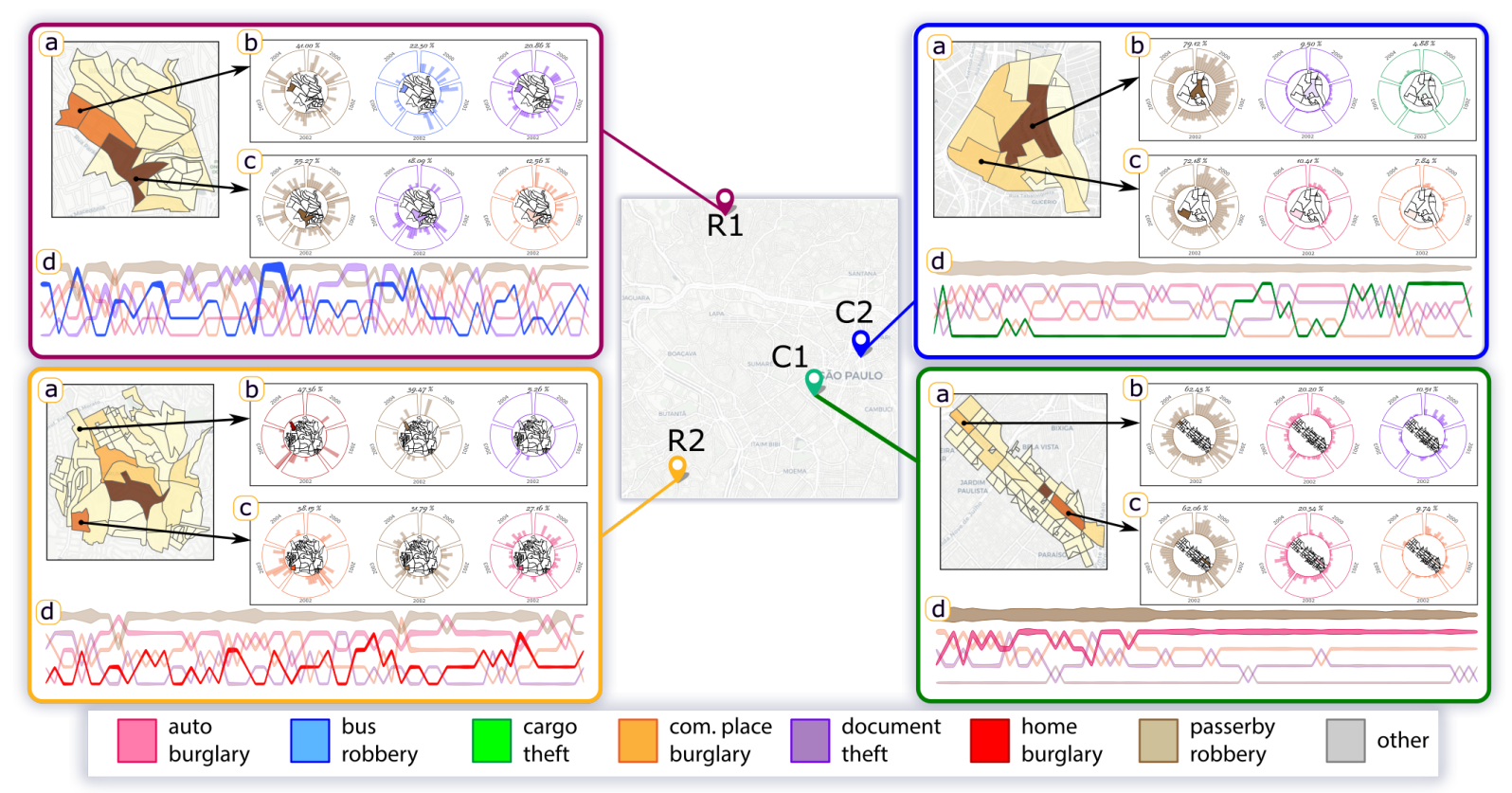

Source: Garcia-Zanabria et al. (2019).

\subsubsection{Comparing Crime Patterns over the City (T1, T2, T3)}

The goal of this case study is to analyze pattern of crimes in different regions of the city in order to understand how they change according to urban characteristics. Moreover, we also investigate the temporal evolution of crime patterns in different regions.

To perform the study we selected four areas in São Paulo, two in the center of the city, denoted as $\mathrm{C} 1$ and $\mathrm{C} 2$ in Figure 17, and two in residential areas, pointed as R1 and R2 in Figure 17. $\mathrm{C} 1$ is a financial district, hosting the headquarter of important banks and financial institutions, while $\mathrm{C} 2$ is a commercial area with many stores, an important metro terminal, and also several touristic places. Both $\mathrm{C} 1$ and $\mathrm{C} 2$ have a huge flow of people during the whole year. Residential areas R1 and R2 differ in terms of the economic level of residents, R1 is a middle-class neighborhood while $\mathrm{R} 2$ is a richer area, with luxurious buildings and houses.

Figure 17 bottom right depicts region $\mathrm{C} 1$, selected by drawing a polyline along the main avenue of the financial district (T1), and highlights the radial type view (C1-c) of the three most prevalent crime types of two sites in $\mathrm{C} 1$ (indicated by the arrows). The ranking type view (C1-d) on the bottom shows how the incidence of the five most frequent crimes varies along the time. Two crime types lead the ranking along the years (the beige and pinkish curves on top), passerby robbery and auto burglary. By analyzing the radial type view (C1-b and $\mathrm{C} 1-\mathrm{c})$ of the highlighted sites, one can notice that those two crime types are indeed the prevalent ones in those regions (encoded by the color). Inspecting other sites by simply clicking on them on the map view, we concluded that passerby robbery and auto burglary are the prevalent crime 
types in almost all sites in C1. It is important, however, that these findings be interpreted in the context of the hypothesis that the spatial distribution of passerby robbery and auto burglary is shaped by the configuration of the street network. Note that the road network in $\mathrm{C} 1$ is the most regular; we can identify this by the number of well-defined city blocks in the analysis area. A city block with this characteristic is common in more consolidated and central urban areas, which leads us to conjecture a relationship between urban infrastructure and burglary risk.

Performing the same analysis in region C2 (top right in Figure 17), which was selected by clicking and expanding the central site of the region (the brownish one), we observe a different behavior. The ranking type view (C2-d) shows that there is one crime type that has been grown over the years (green curve), cargo theft. Selecting cargo theft from the radial type view in the CrimAnalyzer interface (Figure 16(h)), the map view (Figure 16(b)) reveals that cargo theft is not prevalent in the whole region, but it is concentrated in just a few sites, being the dark brown site in the center of the region. Notice that cargo theft became the third most common crime type in that region over time, being behind only of passerby robbery and document theft. Other sites present a more uniform behavior, having passerby robbery, auto burglary, and commercial establishment burglary as the main crime types.

Moving from the city center to more residential areas, the analysis reveals a substantial change in crime patterns, as one can observe on the left of Figure 17, where the crime pattern in R1 and R2 is summarized. In the residential region R1, for example (top left in Figure 17), passerby robbery remains the most common crime type, followed by document theft. However, some sites in R1 have bus robbery (passengers and/or drivers of public bus service are robbed) as the second most common criminal activity (R1-d). The orange site pointed out by the top arrow is an example (R1-b). Site-by-site crime pattern analysis is easy to perform with CrimAnalyzer, in this case, since the number of sites is mild and users need only to select the site on the map to make its crime pattern revealed. The importance of bus robbery in $\mathrm{R} 1$ is easily noticed in the ranking type view (R1-d) depicted on the bottom, where the blue curve (bus robbery) reaches high-rank levels in several opportunities.

Similarly to what happen in C1, C2, and R1 (and also in most of the city), region R2 (bottom left in Figure 17) does have passerby robbery as the predominant crime type, what can clearly be seen from the ranking type view (R2-d). However, crime patterns vary considerably among the sites, and some of them do not even have passerby robbery as the prevalent crime, as the two highlighted sites, which have passerby robbery as second in importance (R2-c and R2-d). Moreover, home burglary is the most relevant crime in one of those regions. In fact, home burglary is a relevant crime in $\mathrm{R} 2$ as a whole, as indicated by the reddish curve in the ranking type view (R2-d). Notice that home burglary has increased in importance over the years.

The discussion above shows that the visual analytic functionalities implemented in CrimAnalyzer are able to sort out analytical tasks T1, T2, and T3 in a simple, intuitive, and 
effective way. The flexibility to handle spatially complex neighborhoods with different shapes allows users to scrutinize set of blocks as well as regions along avenues and streets (analytical task T1). The combination of the ranking type view and the radial type view allows users to understand crime pattern in each region and in particular sites, making evident how crime patterns change around the city and even from site to site in a particular region (analytical task T2), a task difficult to be performed without the our visualization infrastructure. In particular, ranking type view and radial type view turn out to be effective in revealing the temporal behavior of crime patterns, making clear that patterns have changed along the years (analytical task T3). With the provided visual resources, this analysis would be an arduous process, demanding the implementation of multiple filters and sophisticated numerical and computational tools. In fact, the difficulty in performing a similar analysis with existing analytical systems is partly due to limitations on their visual resources and partly to the inadequacy of existing tools to reveal gist information hidden in the data.

Figure 18 - Hotspots around the BR116 and SP230 highways considering all crime types. The highways are highlighted in red and the nearby avenues in blue. The ranking type views below show the five most prevalent crimes of each hotspot.

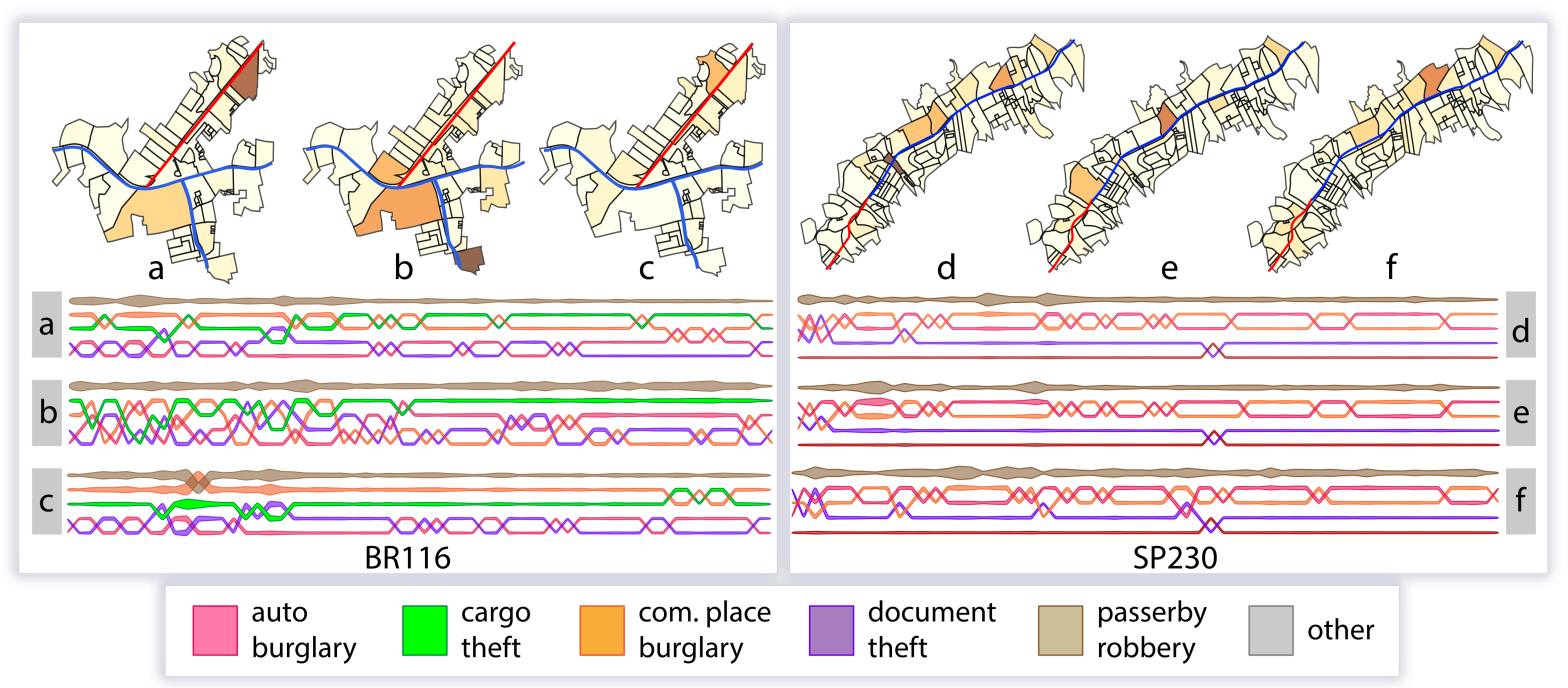

Source: Garcia-Zanabria et al. (2019).

\subsubsection{Hotspot Analysis and Cargo Theft (T1, T4, T5)}

This case study has been driven by the domain experts, and they were interested in a particular type of crime, cargo theft. Although cargo theft does not figure among the most prominent crime types in São Paulo, it is of great interest due to its spatial characteristic, the high values involved, and the engagement of violent gangs in this type of criminal activity. It is well known that robbery (or theft) of high valuable cargo commodities tends to happen close to the main highways connecting São Paulo to other regions of Brazil. Therefore, domain experts 
Figure 19 - Cargo theft hotspots along two important highways, BR116 and SP230. BR116 presents a much larger and more frequent number of cargo theft than SP230.

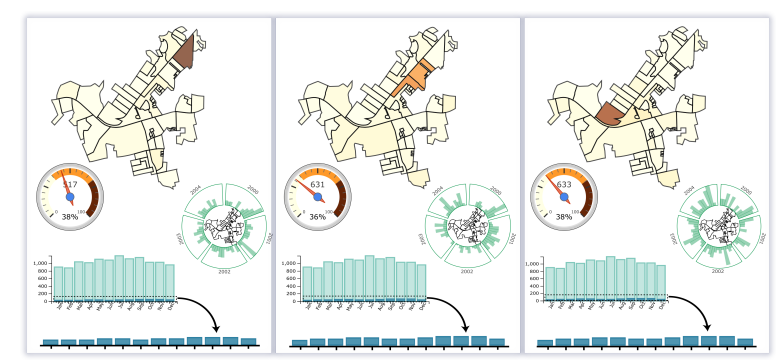

(a) BR116

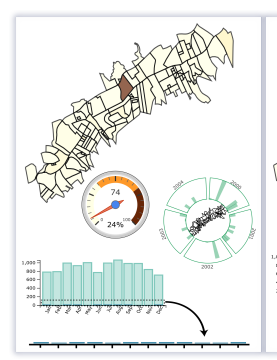

Source: Garcia-Zanabria et al. (2019).

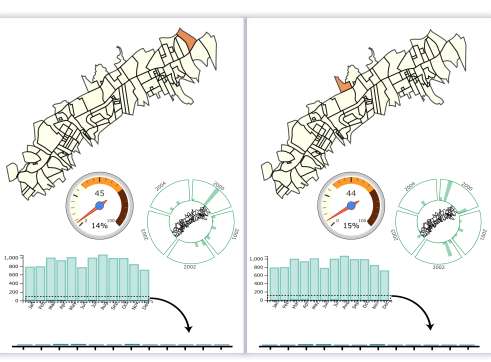

(b) SP230

focused their analysis in two important highways, SP230, which connects São Paulo to states in the south of Brazil, and BR116, which connects São Paulo to Rio de Janeiro.

In order to perform their analysis, domain experts relied on the polyline selection tool to select a considerable number of sites along the highways and avenues that connect the city to the highways. The number of regions involved in these analysis renders a site-by-site investigation tedious, making hotspots a better alternative. Figure 18 shows three hotspots obtained from the regions selected along BR116 and SP230 and nearby avenues. The highways are highlighted in red and the nearby avenues in blue in the hotspot maps depicted in Figure 18. The ranking type view reveals the crime patterns in each hotspot (considering only the five most relevant crime types). Notice that in BR116, cargo theft figures among the most relevant crimes (green lines), becoming the second most relevant crime at multiple times. In SP230, though, cargo theft is not predominant, not appearing among the five most relevant crimes in the ranking type view in any hotspot. In SP230, the predominant pattern is passersby robbery, vehicle burglary, and commercial establishment burglary. CrimAnalyzer makes clear which sites are relevant in each hotspot, their crime patterns, and how crime patterns evolve, thus properly addressing analytical tasks T4 and T5.

However, the experts are interested in cargo theft. To center the analysis in a single crime type users only need to select that type in the radial type view, filtering the data such that hotspots and all the views are updated to depict only information related to the selected crime type. Figure 19 shows the hotspots associated to cargo theft only. The gauge widgets show that the number of cargo theft in the BR116 is one order of magnitude larger than in SP230, also presenting a higher rate of occurrence. The temporal evolution (radial type view) on the center-right of each grid shows the temporal behavior of cargo theft in each hotspot. It is clear that the number of cargo theft in SP230 has lessened over the years, while in BR116 no reduction is observed. The histograms below the gauge widget show the intensity of cargo theft (the short dark bars) in each month, comparing them against the total number of crimes in the region. 
Figure 20 - Commercial establishment burglary tends to increase during the winter (winter in South America goes from mid June to mid September).

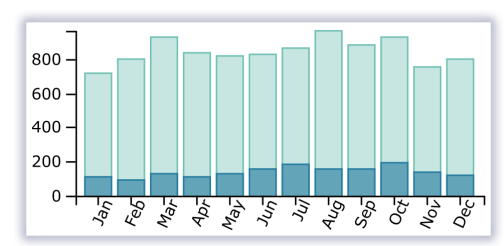

(a) Commercial dist. 1 .

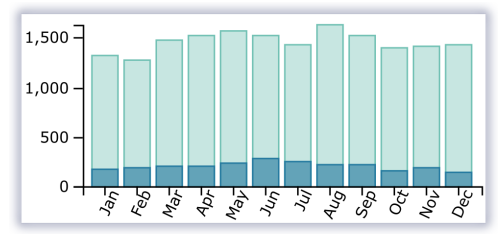

(d) Commercial street 1 .

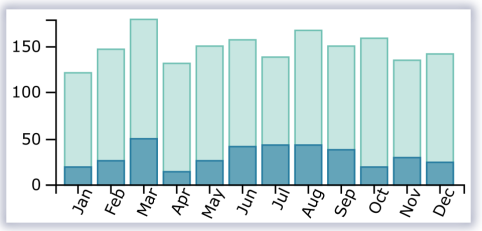

(b) Commercial dist. 2.

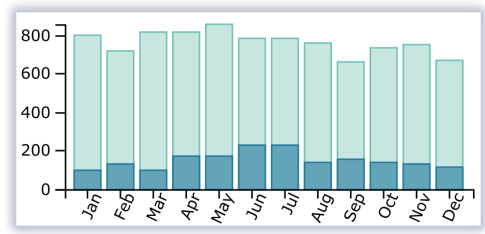

(e) Commercial street 2.

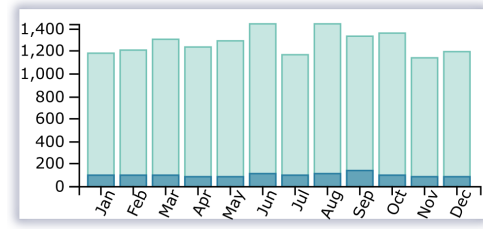

(c) Commercial dist. 3 .

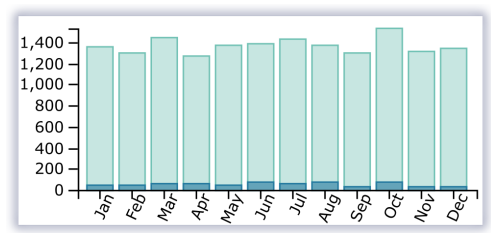

(f) Commercial street 3 .

Source: Garcia-Zanabria et al. (2019).

The CrimAnalyzer viewing tools also make clear that, in BR116, cargo theft takes place mainly along the highway (red curves in BR116 maps in Figure 18), while in SP230 the relevant sites of each hotspot are located in the avenue that connects the highway to the city (blue curves in SP230 maps in Figure 18). Domain experts considered this an important finding because it is known that the modus operandi of criminal offenders and, hence, the location of Cargo Theft change according to the transported product. So, the possibility of identifying these roads should make public security policies more efficient. Another interesting aspect pointed out by the experts is the capability of revealing hotspots associated with sparse criminal activities, as the one depicted in Figure 19b (see the spikes in the radial view). Sparse hotspots are relevant and deserve to be investigated, as they may be associated with local characteristics that would likely increase the chance of crimes being committed. Notice that these findings could hardly be made without the visual resources enabled by systems such as CrimAnalyzer.

\subsubsection{Seasonality and the Temporal Element of Crime (T3)}

This case study corroborates whether some criminal behaviors described and validated in previous works also take place in São Paulo.

Seasonality An important aspect related to criminal activities is seasonality. There is a number of studies in the literature that support the hypothesis that certain crime types are seasonal while others are not. For instance, van Koppen and Jansen (KOPPEN; JANSEN, 1999) argue that, in Netherlands, commercial establishment burglary (robbery) increases during the winter due to the increased number of dark hours during the day. In South America, winter usually starts in mid-June and last until mid-September, during this period, especially in July and August, the number of dark hours is higher than in the rest of the year. An interesting question related to task T3 is whether the findings of van Koppen and Jansen is valid in São Paulo. To look for an 
Figure 21 - The Near Repeat Victimization phenomena. When a home is burgled, the risk of recidivism in a short period of time is not only higher for the targeted home, but also for the nearby homes.

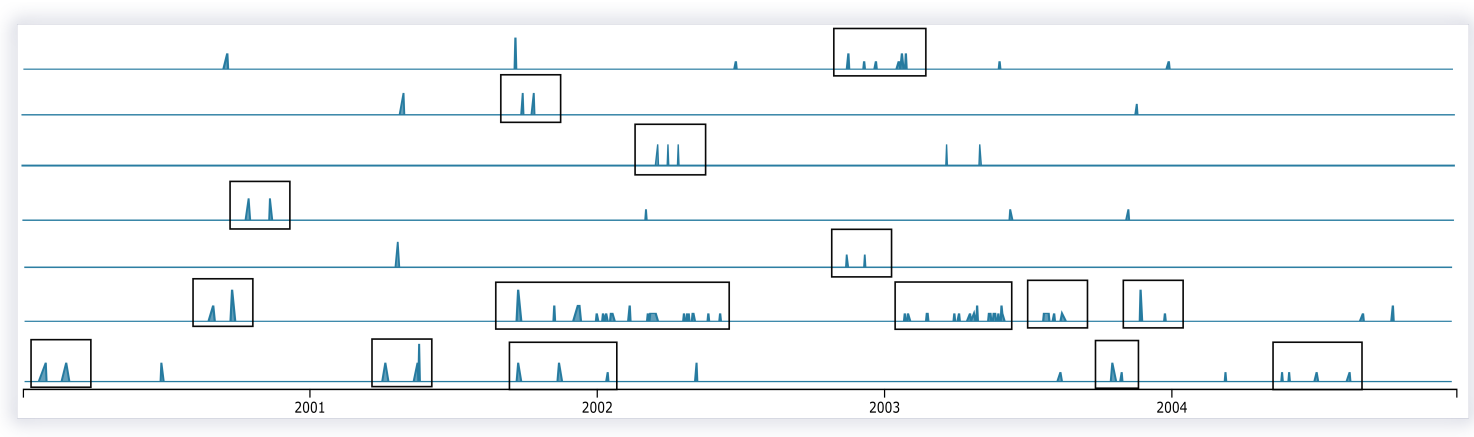

Source: Garcia-Zanabria et al. (2019).

answer, we relied on CrimAnalyzer to explore six major commercial areas in São Paulo city, three commercial districts and three popular commercial streets. Figure 20 shows the cumulative temporal view of each of the analyzed regions. The overlaid darker histograms correspond to the number of commercial establishment burglary and robbery in each month. The overlaid histogram is generated by simply selecting commercial establishment burglary in the temporal type view.

From Figure 20 one clearly sees that five out of six regions present an increase in the number of commercial establishment burglary and robbery during the winter (a-e), thus supporting the findings of van Koppen and Jansen. Although we can not claim with certainty that the hypothesis is true, the analysis enabled by CrimAnalyzer provides evidence about the seasonality of this type of crime, thus helping to answer one of the questions associated to task T3.

Near Repeat Victimization Near repeat victimization theory claims that when a home is burgled, the risk of recidivism is not only higher for the targeted home, but also for the nearby homes, with risk period that seems to decay after some weeks or months (POLVI et al., 1991). The near repeat victimization theory has found evidence of its veracity in a number of countries, but we could find no report about it in São Paulo.

Using CrimAnalyzer, we scrutinized two regions in São Paulo where home burglary is a recurrent crime, including region $\mathrm{R} 2$ discussed in the case study presented in Section 4.6.1. Figure 21 shows the time series, in a daily temporal scale, of seven sites in the analyzed regions, which varies in terms of the frequency of crimes and the number of home burglary. The boxed spikes point home burglary events that occur less than thirty days apart from each other.

Notice that even in sites where home burglary is really occasional (rows 2 to 5 in Figure 21), the near repeat victimization phenomena can clearly be observed.

Seasonality and Near Repeat Victimization are straightforward to be observed with CrimAnalyzer, enabling a number of analytical possibilities. For instance, in warmer seasons, 
day light lasts longer, encouraging a larger number of people to stay on the streets, increasing their exposure to illicit acts and criminal activities. During holiday season, it is common for people to travel to the countryside, leaving their property unprotected, facilitating burglary and other forms of crime. Those phenomena can also be analyzed with CrimAnalyzer.

\subsection{Evaluation from the Experts}

After using CrimAnalyzer and running a variety of experiments, including the case study reported in Section 4.6.2, the domain experts have given us the following feedback.

"Despite its limitations, CrimAnalyzer has allowed us to better understanding challenges not yet elucidated by conventional crime analysis tools. First, by using solid mathematical and computational resources to reveal geo-referenced criminal activities, CrimAnalyzer incites the search for plausible explanations for the observed criminal patterns, what would be impossible with conventional analysis. Second, CrimAnalyzer motivates reflection about the relationship among the different crime types and about topological, directional, and relational connections that might affect the number of crimes in specific locations and time intervals. Third, an analytical tool that enables the analysis of crimes in specific locations leads to thinking the city in its complexity and, at the same time, guides the investigation of urban characteristics (administrative, demographic, physical, and social) and their interaction from which the observed local patterns result. Fourth, CrimAnalyzer uncovers the heterogeneity of the city as to its urban infrastructure, the differences among commercial, financial, and residential areas, the flow of people, public and private transportation, as well as the need for improvements, not only in terms of policing in specific locations and according to the type of crimes, but also, and mainly, in terms of tools to assist criminal investigation towards reducing the high rates of impunity. Finally, in contrast to more simplistic statistical methodology, the deterministic approach for hotspot identification turns out fundamental to emphasize the dynamics of spatio-temporal processes and to capture typical social manifestations such as crimes."

The experts were quite enthusiastic about CrimAnalyzer, as it allowed them to understand and raise hypotheses about a number of phenomena, as in the cargo theft case, that would be hard otherwise. Specifically, one of the experts said: "Analyzing the vast amount of information enabled by CrimAnalyzer, we could detect spatio-temporal patterns and trends that will allow us to improve public policies...".

\subsection{Discussion and Limitations}

CrimAnalyzer was developed in close cooperation with domain experts. The current version satisfies their requirements, however, some limitations and future work have been identified as part of our collaboration. 
NMF stability. Our approach for identifying hotspots is not stable, this is because the NonNegative Matrix Factorization technique depends on the initial conditions of the optimization procedure. To counteract this effect, some implementations, like the one we are using in our system, enables us to run the method a number of times, keeping the solution with the smallest error. Although the results get quite stable after enabling the multiple run alternative, a more robust approach could be sought to mitigate possible effects.

Space Discretization. The space discretization used in CrimAnalyzer is the census units in São Paulo, we adopted this measure because our collaborators had an interest in seeing the analysis in this level of detail. However, we are aware of the modifiable areal unit problem (MAUP), census units do not represent "natural units" of analysis and the result of certain analysis can change by modifying the aggregation unit (CHAINEY; RATCLIFFE, 2005). An immediate future work would be to extend and make more flexible our space discretization. In this way, we should be able to apply our tool in other scenarios.

Multiple data sources. Crime events by their own rarely tell the whole story. Additional data that can be used to enhance the understanding of the crime layer. For example, the presence of bars and pubs, distance to parks, vacant land and buildings, weather, among other information might have a relation with certain criminal activities. Given the increasing number of initiatives to make data publicly available, we are considering to combine that information to further understanding crimes in urban areas. An interesting mathematical tool in this context is tensor decomposition, a generalization of matrix decomposition able to extract patterns from multiple data sources. Developing visual analytical tools to map tensor decomposition information into visual content is an important problem (BALLESTER-RIPOLL; LINDSTROM; PAJAROLA, 2019) that has barely been approached in the context of crime analysis.

Global vs Local approach. CrimAnalizer uses a local-based approach to explore and analyze crime patterns. Even though this was a requirement from the domain experts, and we agree that it was the correct approach to this problem, mainly because domain experts have prior knowledge and hypothesis regarding crime behaviors in particular locations, in some of our interviews with domain experts we discussed the option of having a global-based technique that might process the whole space and propose interesting locations to be explored. This alternative was accepted by the experts but as a complementary technique. As future work, we are also interested in tackling this problem from both perspectives (global and local).

Multiple cities and different scenarios Finally, we applied and validated our system in other cities and countries. Currently, we are in the process of collecting crime data from multiple locations, and in a short time, we expect to release the system to analyze multiple cities. In addition, our approach can be extended to other scenarios than crime analysis. For instance, one can use the system to analyze the dynamics of traffic-accidents, making possible to uncover how the number of car-car crashes, car-bus crashes, run overs, etc. evolve over time. 


\subsection{Final Considerations}

In this chapter, we have described CrimAnalyzer, a visualization assisted analytical tool to support the analysis of crime in local regions. This tool has been developed in close collaboration with domain experts and translated their analytical into procedures the visualization system. We also propose a technique based on NMF to identify hotspots. Qualitative and quantitative comparisons validated our system and case studies using real data and feedback from domain experts. Moreover, we verified two crime behavior (i.e. seasonality and near repeat victimization) using São Paulo crime data.

With CrimAnalyzer and NMF, we obtained interesting results, but there are some additional requirements or drawbacks. For instance, the space discretization used in this work is the census units in São Paulo City. We adopted this measure because of the data set (Data Set 1- see Section 3.3.1). However, we are aware of the Modifiable Areal Unit Problem (MAUP), census units do not represent "natural units" of analysis, and the result of the certain analysis can chance by modifying the aggregation unit (CHAINEY; RATCLIFFE, 2005). Besides, crimes are mostly concentrated in 'Micro' places that are relatively stable over time (GROFF; WEISBURD; YANG, 2010). Therefore, any fine-grained crime analysis demands a street-level discretization difficult to be reached with grids (WEISBURD; MORRIS; GROFF, 2009). In Chapter 5 and Chapter 6 , we will present two visual methodologies that analyze the crime in a street-level of detail.

Another aspect of crime analysis is local or global analysis. CrimAnalyzer uses a localbased approach to explore and analyze crime patterns. Even though this was the requirement from the domain experts because they have prior knowledge and hypothesis regarding criminal behavior in particular locations, a more global analysis, mainly focused on identifying regions with similar crime behavior, is also of interest. Chapter 6 presents a crime analysis technique considering the whole space of study. 
CHAPTER

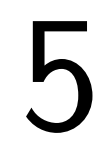

\section{MIRANTE: INTERACTIVE MONITOR FOR CRIME DATA ANALYSIS}

\subsection{Initial Considerations}

An important aspect of crime mapping is the spatial distribution. Most of techniques rely on regular grids with crime data aggregated on grid cells, each possibly covering hundreds of square meters. However, recent studies point out the importance of the analysis of micro places (WEISBURD et al., 2004; BERNASCO; BLOCK, 2011; GROFF; WEISBURD; YANG, 2010), as a crime rarely concentrates on regions larger than a street segment or corner. In fact, several researchers have shown that crimes mostly occur near specific locations such as bars, fast food restaurants, check-cashing centers, and pawnshops, since those places attract distracted and vulnerable people who carry money and valuables (more details about environmental crime opportunities are presented in Section 2.4). In other words, the environment of those places creates a crime opportunity. Therefore, relying on spatial discretizations such as the regular grids renders fine-grained crime analysis a quite challenging task, since the definition of a proper grid resolution and the identification of urban factors impacting the crime opportunity is not so straightforward when crimes are aggregated in a cell containing several street blocks. Even when a small grid resolution is used, the alignment of the grid cell, streets' segments, and other urban structures are not easy to do, hampering the detailed analysis of crime patterns and their possible causes. In addition, the grid representation also limits the analysis of the temporal behavior of crimes. For instance, suppose that a type of crime occurs consistently in a street corner during a period and, after a while, moves to a nearby corner. In a grid representation, such a temporal behavior can hardly be caught if both corners lie on the same grid cell.

In this chapter, we present Mirante, a scalable and versatile visualization tool designed in close collaboration of domain experts that has been tailored to explore crime data in a street-level of detail. Considering street corners as nodes and street segments as edges, Mirante assumes 
city street-network as the spatial discretization. Crime data is spatially aggregated on street corners using an edge-node strategy rather than Euclidean distance, which avoids several issues presented in grid cell aggregation. Mirante provides a number of interactive resources to explore the spatial distribution of crimes and their dynamics over time, making it possible to identify temporal patterns such as the shift of crime hotspots among nearby locations. Interactive filters allow users to focus their analysis on particular hours of the day, days of the week, and months of the year, making it possible to easily scrutinize the seasonality of crimes. Using different selection mechanisms, users can interactively select regions of interest in various scales, enabling the spatio-temporal analysis of large regions as well as quite specific locations of the city, a trait not available in most crime analysis tools. Simplicity and ease of use are other characteristics that render Mirante an interesting alternative in crime mapping.

\subsection{Requirements and Analytical Tasks}

The development of Mirante has been a joint work with a team of sociologists with vast experience in public security and crime analysis. Product of a number of meetings during a couple of years, we raised requirements that guided analytical tasks that are addressed by Mirante.

\subsubsection{Requirements}

During regular meetings with the experts, it became clear their need for mechanisms to set the regions of interest in different scales (i.e., neighborhoods and street segments). In particular, visually identifying street segment or street corners corresponding to hotspots were a major requirement, as their crime mapping tools did not allow them to scale down the analysis to a street-level of detail. Being able to switch the analysis based on the type of crime was also an important requirement. Understanding the spatial dynamics of hotspots and their patterns was important, as the available tools do not enable interactive filtering mechanisms to analyze the behavior of crimes over time. In summary, we point the major requirements as:

- R1 - Selecting regions of variable sizes. Selecting regions of interest with variable sizes while maintaining the ability to perform analysis in a street-level of detail.

- R2 - Identifying high hotspots. Identifying hotspots in a high level of detail in order to trace a relation between urban factors and crime.

- R3 - Switch crime types. Crime patterns depend on the type of crime under analysis. Therefore, being able to switch between different types of crime is an important issue to compare crime behavior in a location of interest.

- R4 - Crime Pattern Analysis. Understanding the dynamics of crime is a fundamental task in crime mapping. Therefore, identifying and exploring crime patterns (in this context, 
crime patterns account for the temporal behavior of crime activities in each particular location) is also fundamental to determine the urban factors that impact the emergence or eradication of crimes.

\subsubsection{Analytical Tasks}

The requirements described above gave rise to a list of analytical tasks that must be accomplished by the visualization tool.

- T1 - Visualize hotspot in a street-level of detail. Enable visualization resources to reveal hotspots located in street corners and street segments. This task is related to requirements $\mathrm{R} 2$ and $\mathrm{R} 4$.

- T2 - Interactive selection of regions of interest. Provide interactive mechanisms to select regions of interest that range from whole neighborhoods to a few set of street segments, enabling analysts to focus their analysis on different levels of details. This task accounts for requirements R1, R3, and R4.

- T3 - Switch crime types in a given region. Filter the analysis of different crime types in a given region. This task achieves the requirement $\mathrm{R} 3$.

- T4 - Show crime seasonality. Allow the exploration of crime seasonality, making it possible to filter crime occurrences according to periods of the day, day of the week, and month of the year. This task accounts for requirements R3 and R4.

- T5 - Identify crime patterns. Explore crimes based on specific time windows, thus uncovering hotspot patterns. This task helps achieve the requirements R3 and R4.

Mirante integrates the tasks above into a meaningful and straightforward visualization tool. Details of Mirante's implementation is provided in the following section.

\subsection{Mirante System}

In contrast to most crime mapping techniques, which rely on regular grids as spatial discretization, Mirante represents the spatial component as a street-network, where street corners correspond to the nodes of the graph and street segments to the edges. Crime data is aggregated in each node of the graph and depicted as a graph-based heatmap when a region of interest is selected. The heatmap is updated according to filters that users can interactively apply to the data. In the following, we detail the construction of the graph corresponding to the spatial discretization, the design of the visual components, their functionalities, and implementation details. 
Figure 22 - Two ways to build a crime-based street-network by closest node based on: (a) Euclidean distance, and (b) edge-node strategy.

(a) Closest node strategy.

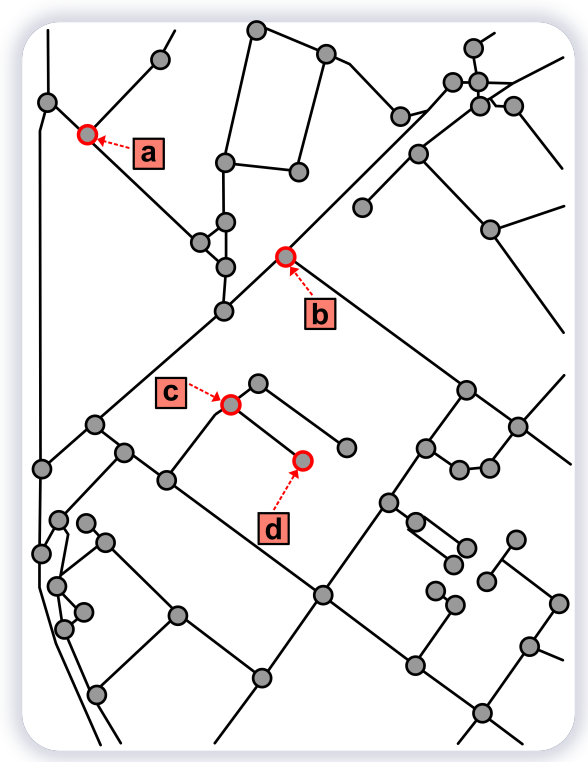

(b) Edge-node strategy

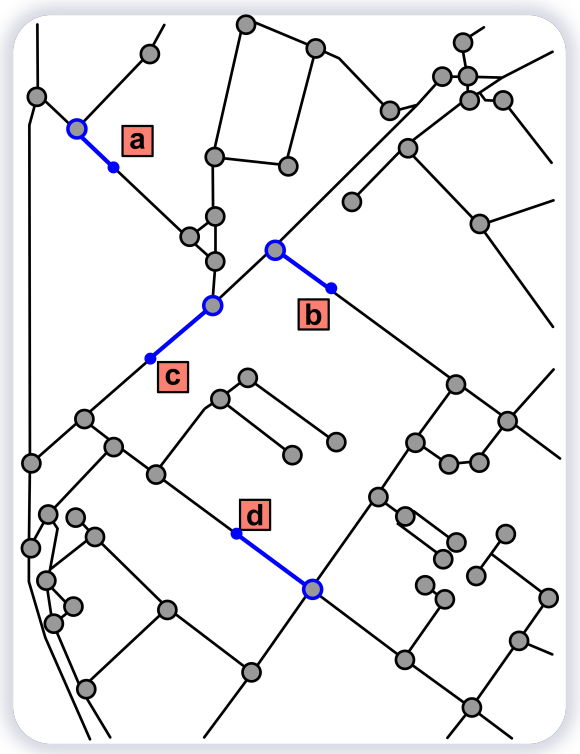

Source: Garcia-Zanabria et al. (2020a).

\subsubsection{Building the spatial representation}

To build the graph corresponding to the spatial discretization, we use the OpenStreetMap API (OpenStreetMap contributors, 2017), which allows for generating a street-graph containing roads and intersections for entire cities. It is possible to define the type of map to use, e.g., pedestrian, bike, and car drive roads. We opt to use the pedestrian map, as it comprises drive roads and pedestrian walkways.

The number of nodes and edges derived from the map varies considerably depending on the city. For instance, the API returns a set of 533,437 vertices and 1, 197, 828 edges for São Paulo city - which we use in some of our experiments. However, a large number of vertices do not correspond to street intersections. To remove non-intersection vertices and all the points along a single street segment, we run a procedure (see (BOEING, 2017) and corresponding implementation included in the OSMnx library) that topologically simplifies the graph. For São Paulo street map, the simplification results in 142, 112 vertices and 415, 178 edges, a considerable reduction that facilitate interactive procedures.

\subsubsection{Assigning data to the nodes of the spatial graph representation}

Let $L_{\text {crime }}=\left\{c_{0}, c_{1}, \ldots, c_{n}\right\}$ be a list of $n$ crime records, where each $c_{i}$ contains information such as record id (unique identifier), location (latitude, longitude), crime type, date, number of people involved, among other information. Let $G=(V, E)$ be the graph corresponding to the city's spatial representation. Each vertex has a unique geo-referenced coordinate (identifier, latitude, longitude), and each edge represents a segment joining two intersections. 
In our context, each crime record $c_{i}$ must be assigned to a vertex of the graph $G$. The easiest solution would be to assign each $c_{i}$ to its nearest vertex using the Euclidean distance. However, using Euclidean distance is not appropriate because it does not consider the topology of the spatial representation. We illustrated this issue in Figure 22. Notice that using Euclidean distance the crime records "a" and "b" are properly assigned vertices, however, records "c" and "d" are not, since it is clear that the corresponding crimes took place on the street segments closer to them, so they should be assigned to one of the vertices defining the segments. Figure $22 \mathrm{~b}$ shows the correct procedure, which we call edge-node strategy, where first, the nearest edge $\left(e_{\text {near }}\right)$ is found and then the closest vertex. In other words, given $G$ and $L_{\text {crime }}$, for each crime record $c_{i} \in L_{\text {crime }}$ we calculate its nearest edge $\left(e_{\text {near }}=\right.$ G.get_nearest_edge $\left.\left(c_{i}\right)\right)$ and consequently, its end-nodes $\left(\left(v_{1}, v_{2}\right)=G\right.$.get_vertices $\left.\left(e_{n e a r}\right)\right)$. Finally, we compute the distance to both end-nodes $\left(d_{\{1,2\}}=\right.$ greatCircleDistance $\left.\left(c_{i}, v_{\{1,2\}}\right)\right)$ to assign the record to nearest node $v_{1}$.crimes.append $\left(c_{c u r r}\right)$ if $d_{1}<d_{2}$ or $v_{2}$.crimes.append $\left(c_{i}\right)$ otherwise. Algorithm 1 details this procedure.

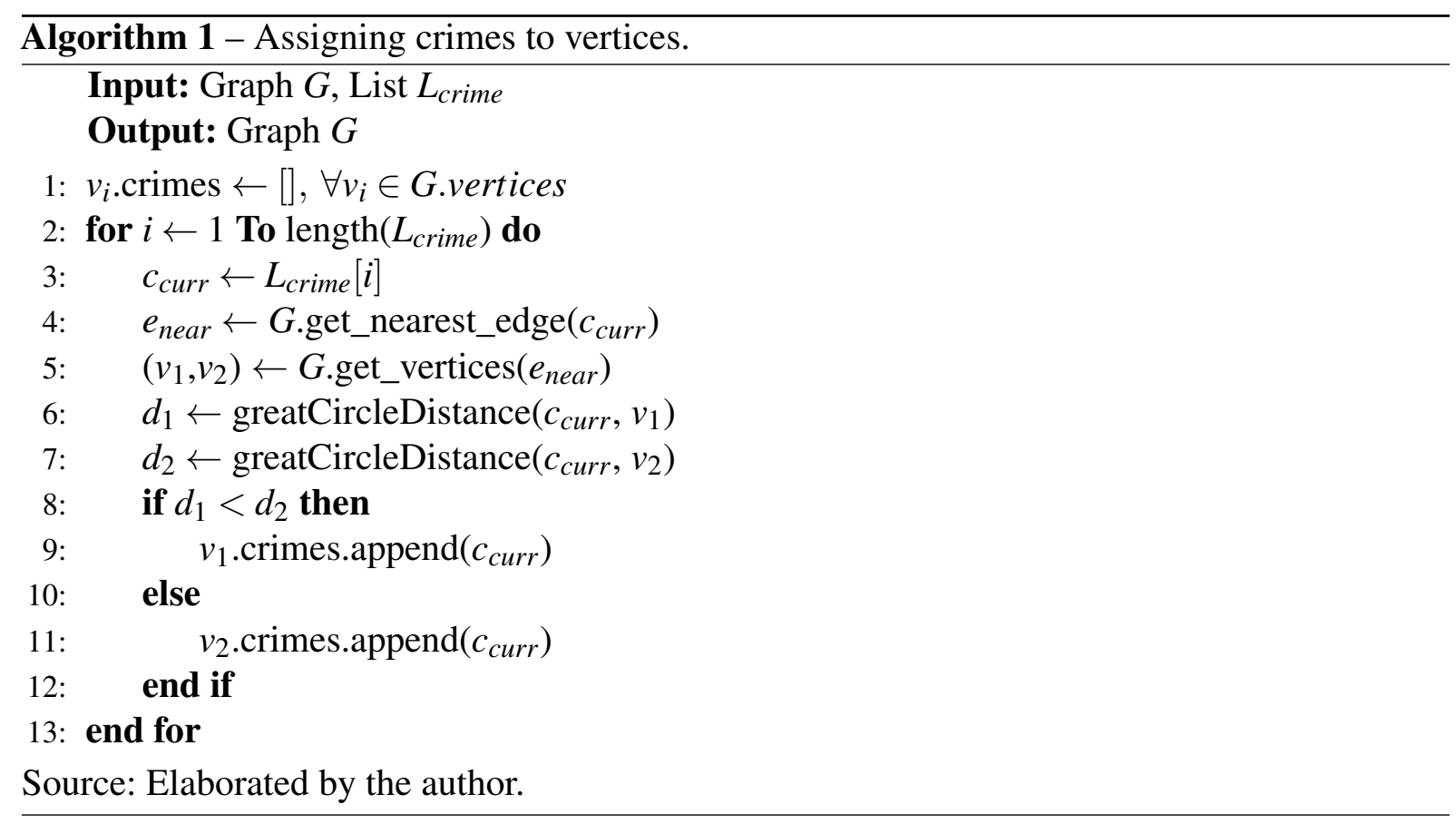

The crime-vertex assignment starts by traversing the list of crime records $L_{\text {crime }}$ to compute their nearest edge $e_{\text {near }}$ in the graph $G$. Different strategies can be used to efficiently perform this step, e.g., using a spatial data structure as Quad-tree or Ball-tree. In our case, we use an R-tree implemented in the OSMnx library $\left(e_{\text {near }}=\right.$ G.get_nearest_edge $\left.\left(c_{i}\right)\right)$. Once the nearest edge $e_{\text {near }}$ and end-nodes $\left(\left(v_{1}, v_{2}\right)=\right.$ G.get_vertices $\left.\left(e_{\text {near }}\right)\right)$ is found for each record, it is assigned to the closest edge node. For that, we compute the distance to both nodes $\left(d_{\{1,2\}}=\right.$ greatCircleDistance $\left.\left(c_{i}, v_{\{1,2\}}\right)\right)$ and crime record in $c_{i}$ are stored into the list crimes associated to each node, that is, $v_{1}$.crimes.append $\left(c_{i}\right)$ if $d_{1}<d_{2}$ or $v_{2}$.crimes.append $\left(c_{i}\right)$ otherwise. List per-vertex is used to temporally aggregate crime records (hourly aggregation in our case), giving rise to a time series associated to each vertex. 
Figure 23 - An overview of the Mirante tool, a set of spatiotemporal visual resources enabling the exploration of crime patterns in a region: (a) Street-level Heatmap, (b) Temporal Evolution View, (c) Temporal Histogram View, (d) Selector toolbox, (e) Address Search Bar, (f) Evolution Animation Controller, and (g) Local/global crime ruler.

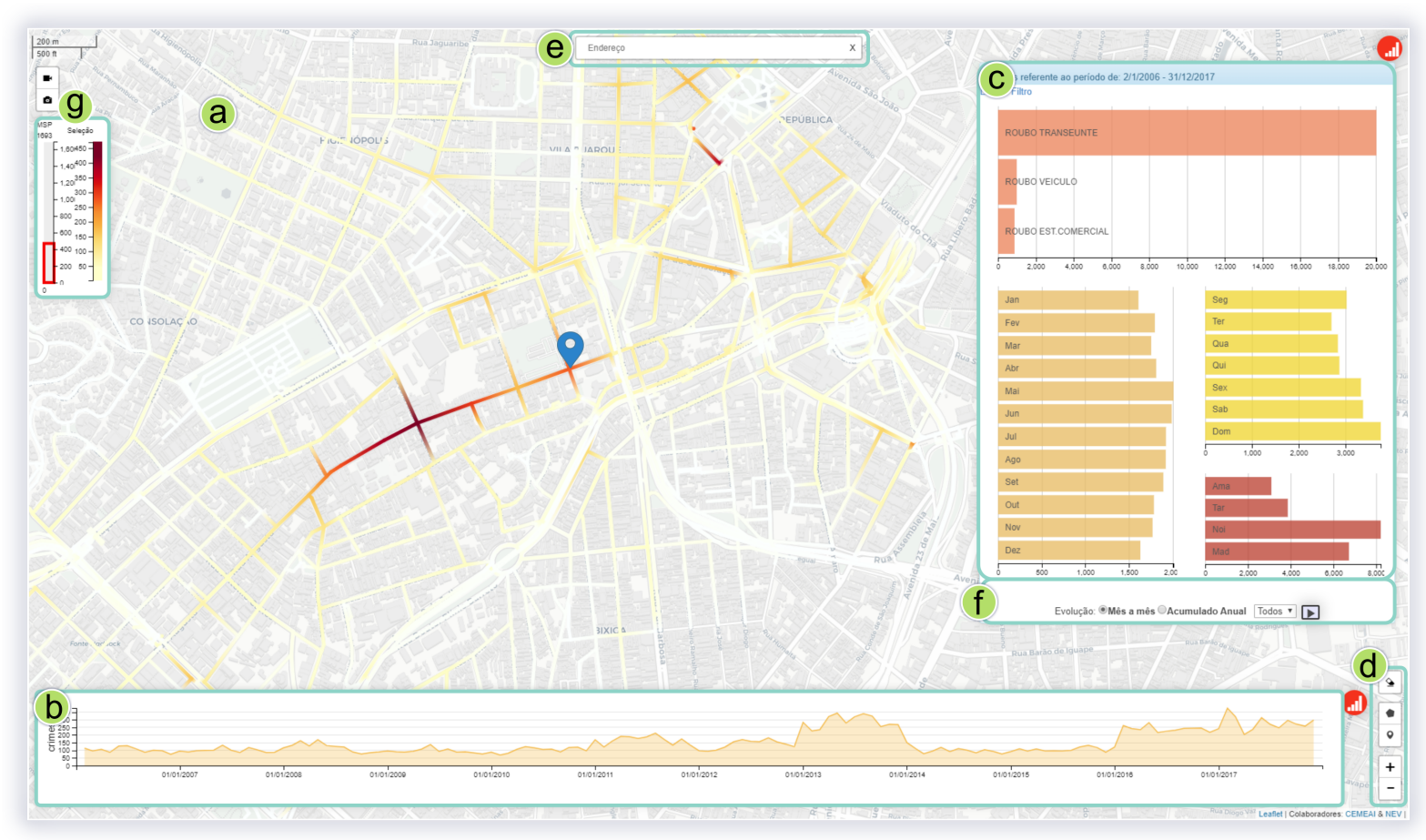

Source: Garcia-Zanabria et al. (2020a).

To alleviate memory usage, we store the graph and node time series into a PostGISPostgresSQL database, allowing us to perform querying/retrieving operations quite efficiently.

\subsection{Visual Components}

Mirante brings a set of fully-linked visual components for supporting the exploratory analysis of crime data in a selected region. Figure 23 shows the Mirante system and its seven main visual components:

(a) street-level heatmap to visualize the crime hotspots in a selected region;

(b) temporal view showing the time evolution of a particular type of crime;

(c) seasonal histogram view depicting the seasonality of crimes by a period of the day, day of the week, and month;

(d) selector toolbox that enables different approaches to select regions of interest;

(e) a search bar to select regions of interest based on addresses;

(f) an evolution animation controller; and

(g) a local/global crime ruler. 
Table 4 - Methodological and visualization properties and their analytical tasks presented in Sec.5.2.2.

\begin{tabular}{cccccc} 
& T1 & T2 & T3 & T4 & T5 \\
\hline \hline Street-level heatmap View & $\checkmark$ & $\checkmark$ & & $\checkmark$ & \\
Temporal Evolution View & $\checkmark$ & & & $\checkmark$ & $\checkmark$ \\
Temporal Histogram View & & & $\checkmark$ & $\checkmark$ & $\checkmark$ \\
Selector Toolbox & & & & $\checkmark$ & $\checkmark$ \\
Address Search Bar & $\checkmark$ & $\checkmark$ & & & \\
Evolution Animation Controller & & & $\checkmark$ & $\checkmark$ & $\checkmark$ \\
Local/global crime ruler & & $\checkmark$ & $\checkmark$ & & \\
\hline \hline Source: Garcia-Zanabria et al. (2020a). & &
\end{tabular}

Source: Garcia-Zanabria et al. (2020a).

We design the visual components to fulfill the requirements raised from the interaction with domain experts, thus addressing the analytical tasks described in Section 5.2.2. Table 4 details the relation between the visual resources (first column) and tasks (T1-T5 columns).

\subsubsection{Street-Level Heatmap.}

This view, depicted in Figure 23 (a), seeks to summarize the distribution of crimes across the city by displaying a set of colored segments on streets using the number of crimes assigned to each vertex of graph $G$. For each vertex, we match a color from a palette depending on its stored value, as illustrated in Figure 24a. Given the discrete nature of our data, we use a sequential scale — starting in dark red to light yellow — taken from ColorBrewer 2. $0^{1}$. Then, we use linear interpolation for coloring the lines representing the edges, as shown in Figure 24b.

Figure 24 - Street-level heatmap construction in two steps: Color assignation and linear interpolation.

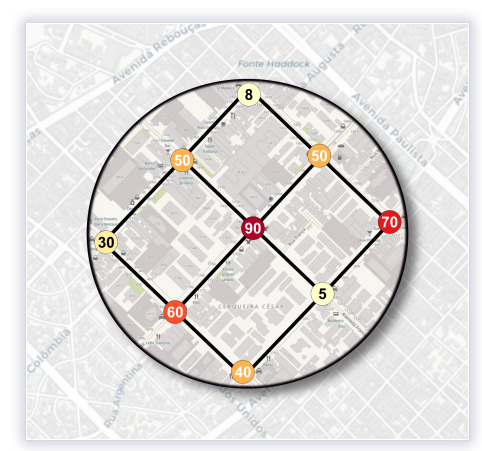

(a) Match of color with crime in-(b) tensity (number of crimes) for each node.

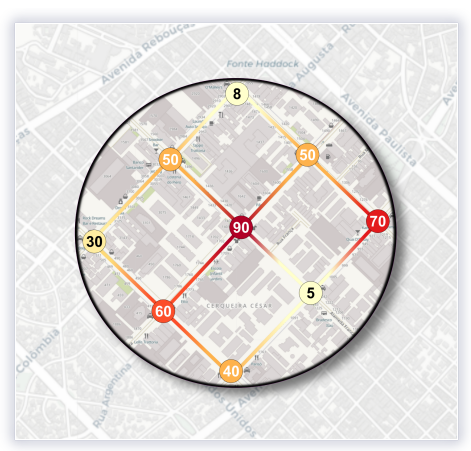

(b) Linear interpolation for coloring all edges between each two adjacent vertices

Source: Garcia-Zanabria et al. (2020a).

\footnotetext{
$1<\mathrm{https}: / /$ colorbrewer2.org/>
} 


\subsubsection{Temporal Evolution View.}

This view uses an interactive line chart for displaying how crime events evolve over time in the selected region, as shown in Figure 23 (b). By default, each tick on the x-axis represents one year of data. Moreover, this view can filter the analysis to focus on a specific range of time, which can be selected by dragging a range window along the $\mathrm{x}$-axis. Once a new range of time is selected, all linked views are updated accordingly.

\subsubsection{Temporal Histogram View.}

It comprises four bar charts that detail the crime distribution in the period of the day, day of the week, and months of the year, as depicted in Figure 23 (c). On the top, the red bars show the volume of crime types in the selected region. On the bottom, on the left histogram (orange bars) show data aggregated by the month, while on the right, the bar charts visualize data by day of the week (in yellow) and the period of the day (in dark red). Each bar chart serves as a filter, that is, if a user clicks on one of the bars, all views are updated to show only crime occurrences in the selected filter. For instance, clicking the bar corresponding to January, only crime crimes that took place in January are depicted in all views.

\subsubsection{Selector Toolbox.}

We implement two different types of area selection: i) Radial-centric area selection, which sets a geographical coordinate as the center and allows the user to interactively define a radius in meters (see Figure 25a). ii) Polygonal area selection that allows users to draw a polygon enclosing a region of interest (see Figure 25b). The selectors are in the bottom-right part of the screen, as shown in Figure 23 (d).

Figure 25 - Two different types of selection of the region of interest.

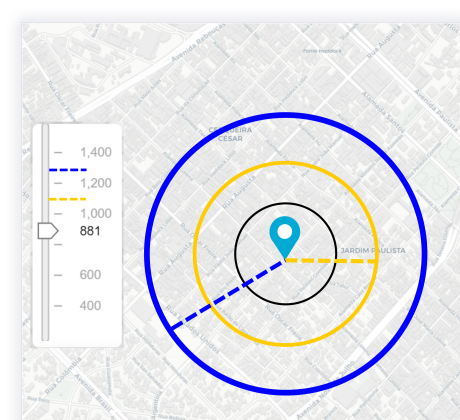

(a) Radial-centric area: Selection of a region based on its center and a radius.

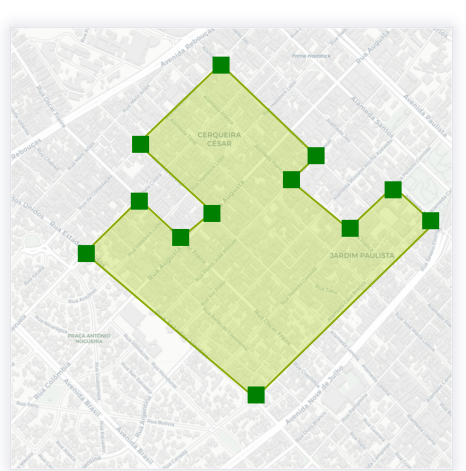

b) Polygonal area: Selection of a region drawing a polygon in a region. 


\subsubsection{Address Search Bar.}

Users can also define a region of interest based on the address search bar that uses Google GeoSearch Engine ${ }^{2}$ to query for the address that most closely matches the one typed by users, as depicted in Figure 23 (e). Once retrieved, the system automatically defines the region of interest as disk centered in the given address.

\subsubsection{Evolution Animation Controller.}

Mirante allows the analyst to inspect, through an animation, how data evolves over the months of the year. The analyst can choose to animate by month separately or cumulatively (see Figure 23 (f)). Figure 26 illustrates four different months displayed automatically by the system, namely, January, March, August, and December during the period 2006 - 2017. Each time-series reveals different crime behavior in the chosen geographical location, which are also shown as heatmaps (top images).

Figure 26 - Four months depicted by the evolution animation.
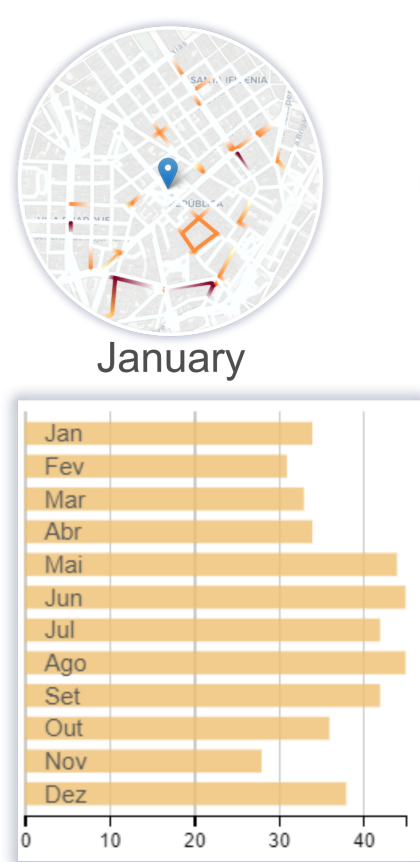

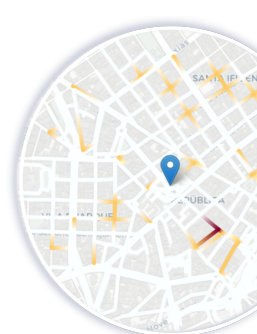

March

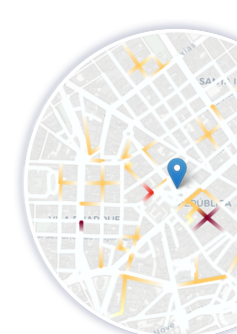

August

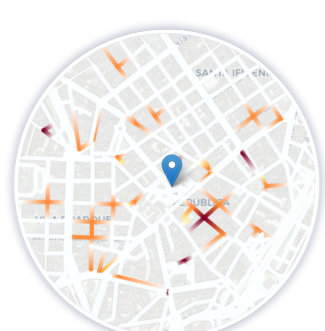

December
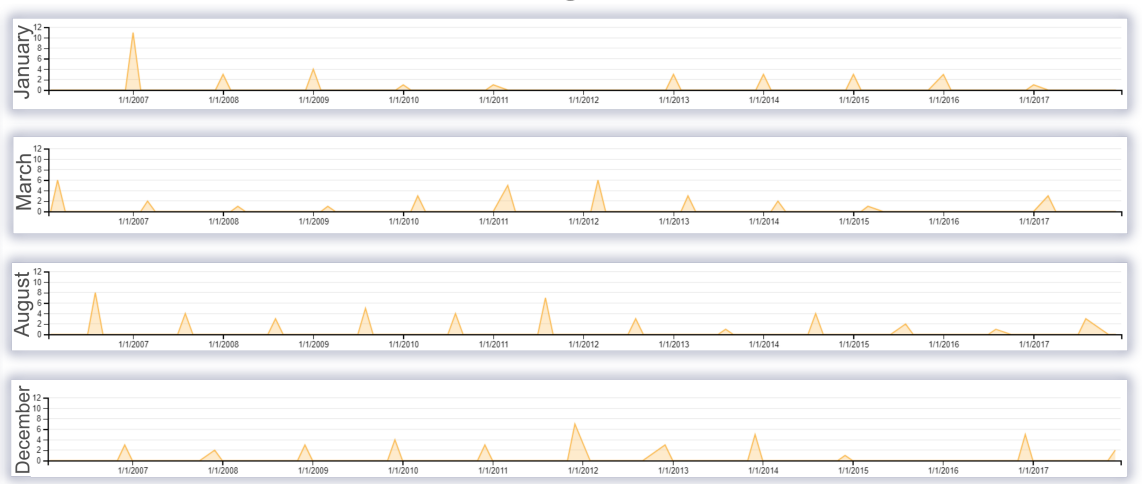

Source: Elaborated by the author.

\subsubsection{Local/Global Crime Ruler.}

One of the main issues when performing local analysis is to figure out how criminal activity observed in the region of interest compares with the amount of crime recorded in the city. To tackle this issue, we use two rulers, the global one that shows how the more intense hotspot of the selected region compares against the more intense hotspot in the whole city, and

$2<$ https://github.com/smeijer/leaflet-geosearch> 
the local ruler that shows the color map used to highlight hotspots in the selected region (see Figure $23(\mathrm{~g})$ ). The rulers allow users to figure out how "dangerous" it is the region of interest compared with the whole city.

\subsection{Implementation Details}

Mirante is a web-based application implemented under the Django ${ }^{3}$ framework. The core of the system comprises data modeling and visualization modules. For the street network, we used OSMnx ${ }^{4}$ and NetworkX $\mathrm{X}^{5}$ python libraries to process data. We achieve interactive rates in most of the calculations by pre-processing the data (i.e., computation of the nearest edges, vertex nodes, and street network simplification). All visualization resources have been developed using JavaScript libraries: Leaflet ${ }^{6}$, to perform the interpolation geo-map and D3.js ${ }^{7}$ to represent line and bar charts. In our case, we have developed extra components to manipulate filters by using Crossfilter ${ }^{8}$ and Dc.js ${ }^{9}$ libraries.

\subsection{Case Studies}

We conduct two case studies to assess Mirante's performance in terms of effectiveness for pattern identification and navigability. We analyze two types of crimes in two different Brazilian cities with very different characteristics: São Paulo (see Section 3.3.1) and São Carlos (see Section 3.3.3), both located in the state of São Paulo.

\subsubsection{Vehicular Robbery in São Paulo City}

In this first case study, we assess the usefulness of Mirante as to its effectiveness in identifying vehicle robbery patterns in a given region of São Paulo. The main task is to understand the impact of changes in the local urban infrastructure in the crime opportunity.

The analysis aims to support a crime pattern theory that suggests a higher risk of car robbery/burglary is linked to the road infrastructure. Roughly speaking, there is a correlation between the distribution of crimes and the urban road infrastructure (O'MAHONY, 2018).

To accomplish the case study, we select a region in the south part of São Paulo. This region is near a favela, i.e., a neighborhood of low income, and unregulated settlements. Using Mirante, we draw a polygon comprising the streets: Bom Pastor Street, Juntas Provisórias

\footnotetext{
$<$ https://www.djangoproject.com/>

$<\mathrm{https} / / /$ osmnx.readthedocs.io $>$

$<$ https://networkx.github.io $>$

$<$ https://leafletjs.com/>

$<$ https://d3js.org/>

$<$ https://square.github.io/crossfilter/>

$<$ https://dc-js.github.io/dc.js/>
} 
Avenue, and Dois de Julho Street (see Figure 27), which accounts for task T2 (see Section 5.2.2). Known as a region with a large number of vehicle robbery, we focus the study on this type of crime (task T3). The selected region contains approximately 650 occurrences of car robbery in the period 2006 to 2017.

Exploring the Street-Level Heatmap View, we note two hotspots labeled as "A" and "B" on the first row in Figure 27 (task T1). Using the Temporal Evolution View, we filter crimes from 2006 to November 2010. In this period, a single prominent hotspot shows up in the northwestern of the region (left image in the second row in Figure 27). Notice in the temporal view that the number of occurrences decreases at that location in that period, but, from 2011 on, crimes present an increase (tasks T4 and T5), and the new prominent hotspot shows up on the southeast of the region, marked as "B" on the right image in the second row in Figure 27.

In particular, we conduct an empirical analysis examining the urban infrastructure in the surroundings of the hotspots employing Google Street View to retrieve and display photos of those locations in the two-time intervals used in our analysis (see the third row in Figure 27). The photos suggest that the risk of burglary might be related to the parking areas and vertical and horizontal transit signs.

In the first case (hotspot "A"), there is an evident change in the horizontal transit sign (see the third row on the left in Figure 27). Specifically, notice in the second left photo that the roundabout was partially removed that was present in 2010 was removed in 2011 (last photo of 2017), forcing drivers to take specific directions, what could make it harder for criminals to quickly escape to the main avenues that border the region of interest, thus triggering a decrease in the number of car robbery in the hotspot " $\mathrm{A}$ ".

Regarding hotspot "B", notice in the left most photo on the bottom that in 2010 it was not allowed to park cars on the right side of the street, and in 2017, the parking was allowed. The direction where cars were allowed to park goes directly to a main avenue that connects, a hundred meters ahead, to an urban highway, thus making it easy to steal a car and quickly escape down the highway (see on the larger photo on the right in Figure 27 the connection with a main avenue).

\subsubsection{Passerby Robbery in São Carlos}

The second case study involves passerby robbery in São Carlos, a mid-sized city in the interior of the State of São Paulo. São Carlos hosts two major public universities; thus, 30 thousand out of the 220 thousand inhabitants are university students. In a series of meetings with authorities in charge of the public security in the city to demonstrate Mirante's capability, the authorities presented public policies implemented in the city to reduce criminality over the years. Despite the reduction in the number of crimes, mainly passerby robbery, it was not clear to the authorities which actions impacted most in crime reduction. Aiming to give some answers, we 
Figure 27 - Vehicle robbery patterns around São Paulo city: (first-row) overview of Mirante with two hotspots in the street-level heatmap. (second-row) Crime behavior using Temporal Evolution View selecting two periods of time, one from to 1/2006 to 11/2010 and another from 11/2010 to 12/2017. (Third-row) Google Street View images of highlighted nodes in different years and from different angles.

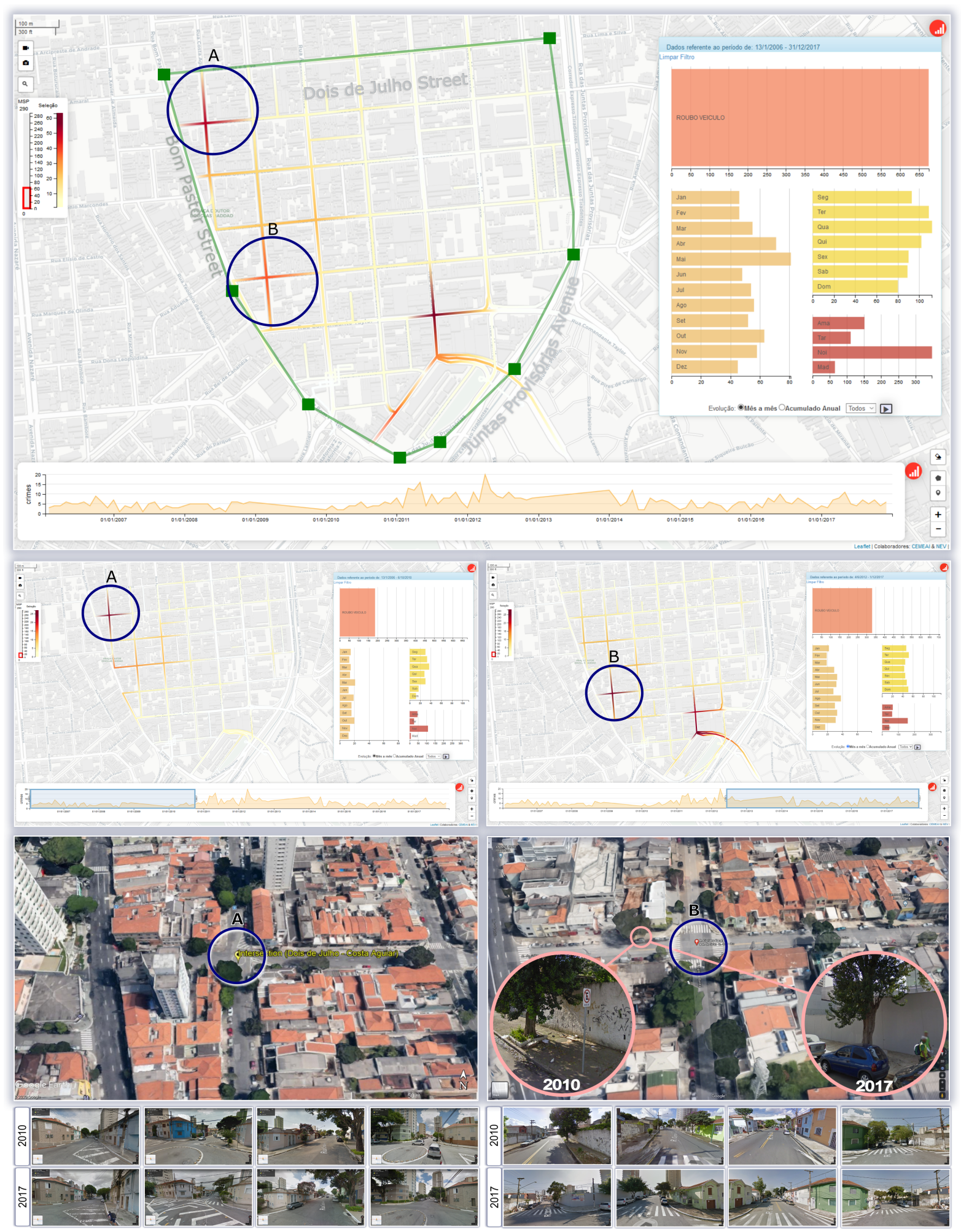

Source: Elaborated by the author. 
Figure 28 - Urban infrastructure impacting in passerby robbery in the city of São Carlos: (first row) Mirante system with a region of interest. (a), (b), and (c) Street-level Heatmap, Temporal Evolution View with selected time intervals, Temporal Histogram View showing the seasonality of crimes, respectively.

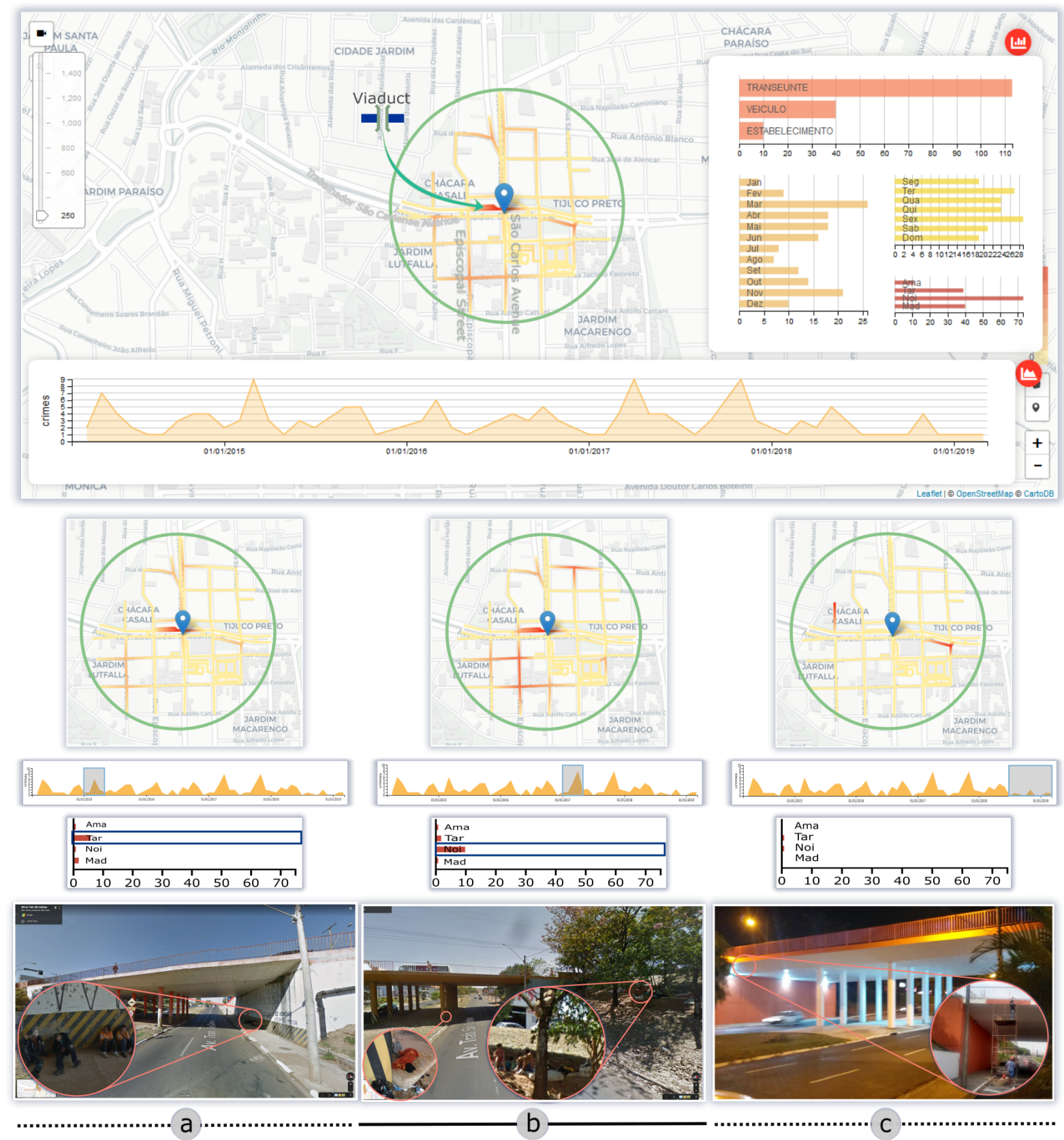

Source: Elaborated by the author.

used Mirante to explore crimes over the city, describing one of our findings in the following.

Specifically, security authorities would like to know whether improvements in the urban infrastructure have any impact on the crime rates, mainly passerby robbery (task T3) in one of the main avenues of the city in the interval from January 2014 to April 2019. Using the selection resource to explore the avenue, one particular site called our attention. Selecting the region of interest using a disk of radius 300 meters (task T2) and choosing three time intervals in the 
temporal view (tasks T4 and T5), we analyzed the behavior of crimes considering the period of the day with the most prevalent crime rate, as depicted in Figure 28. We also use some images from the Google street view to verify which factors could be contributing to the increase or decrease of crimes in each time interval. The top image in Figure 28 shows an overview of the Mirante system with the selected region of interest and the Temporal Evolution View showing the crime evolution over the years. Temporal Histogram View shows that the most prevalent type of crime, months, day, and period of the day are passerby robbery (transeunte in the image), March and November, Friday, and night, respectively. Figure 28 (a, b, and c) show the Street-Level Heatmap (task T1), selected time interval in the Temporal Evolution View, Temporal Histogram View (tasks T4 and T5), and a photo that was taken in the years where the time intervals have been chosen, namely 2015, 2017, and 2019, respectively.

Figure 28 (a)-(Temporal Histogram View) shows that in 2015 crimes were most frequent in the afternoon (blue rectangle). According to the Google Street View photo, no people were living under the viaduct. However, domain experts alerted that during the afternoons, the viaduct became a point where marginalized people used to get together, which can explain the large number of passerby robberies in that period of the day. Figure 28 (b)-(Temporal Histogram View) shows that in 2017 crimes were more frequent in the evening and, according to the Google Street View photo, homeless people were living near the viaduct, which might explain the large number of crimes in the evening. Figure 28 (c)-(Temporal Evolution View) shows a significant drop in crime rates by the end of 2018 and the first quarter of 2019. According to the photos, the region around the viaduct was revitalized in that period (facade, lighting, etc.), making the region safer. Notice that the decrease in the number of crimes is clearly shown in the Street-Level Heatmap. The authorities speculate that the improvements in the infrastructure can explain the decrease in the number of crimes.

São Carlos's security authorities considered Mirante a valuable tool for different types of analysis. In particular, it supported the hypothesis that improvements in infrastructure can help to reduce crime rates in specific locations. They have also pointed out the capability of Mirante to highlight hotspots in a street-level of detail, a functionality they do not have in the crime mapping systems they use to play with. They explicitly said that Mirante is a very interesting analytic instrument that could be quite useful in several scenarios.

\subsection{Expert Feedback}

After using Mirante and conducting the case studies, two experts gave us some feedback. The first paragraph is from the sociologists that used Mirante to analyze crimes in São Paulo (called E1). The second paragraph is from São Carlos's security authorities (called E2).

E1: "The proposed tool has enabled an alternative solution to the challenges we face in our daily analysis. First, modeling the spatial domain in a street-level of detail makes easier 
the understanding of the spatio-temporal characteristics, with good implications for public security and social interrelationships. Second, through visual analysis, Mirante motivates the study of a variety of crimes. Mirante's visual resources make it possible to analyze the dynamics of crimes and their relation with urban factors. Third, each city has its complexity; based on our experience, a city usually brings together diverse places (violent and peaceful). This fact makes global studies less productive due to the lack of local details. The proposed tool enables a focused analysis while preserving the relation between local and global crime rates."

E2: "This research is benefiting our city; it is a critical and unprecedented investigation. Our security professionals already work with crime analysis techniques. However, this crime mapping tool allows a more in-depth and detailed analysis of crime patterns in specific regions. With Mirante's results, we can define actions to reduce crime rates in specific areas. For instance, we could adopt primary interventions such as the improvement of lighting and traffic-flow or police forces interventions."

In both cases, we got positive feedback from the users. The experts explicitly mentioned that their current tools do not manage to do the same analysis as Mirante.

\subsection{Discussion and Limitations}

We constructed the visual resources guided by the requests and analytical tasks of domain experts described in Section 5.2.1. However, there are some limitations and research opportunities identified during the design and implementation processes.

Integration with Google Street View. As evidenced in both case studies, urban infrastructure has an important impact on crime rates. Such a finding was possible due to Google Street View, which was used as a side tool. Integrating Google Street View API with Mirante, would enable a more comprehensive exploratory analysis.

Street-Network Topology. As detailed in Section 5.3, it is possible to make use of different street maps. In this work, we use pedestrian walkway maps to derive the graph that supports our spatial discretization. However, a unique network for various crime types could lead to misinterpretations. As future work, we will implement a resource to allow users to switch spatial representations on the fly.

Multiple Data Sources. A limitation of Mirante is that it only plays with a single source of data, crime data. However, a more insightful analysis could be performed if other sources were made available. For instance, providing mechanisms to enable the joint analysis of crime and flux of people could result in much richer outcomes. How to integrate multiple data sources into Mirante is a challenge we aim to sort out. 


\subsection{Final Considerations}

In this work, we introduced Mirante, a visualization tool tailored for crime data analysis. Mirante relies on street maps as spatial discretization, and it enables a set of fully-linked visual resources to filter crime data based on time intervals and seasonality. Enabling street-level of detail analysis is a particularly important trait of Mirante that is not available in most crime mapping systems. The provided case studies show the effectiveness of Mirante in identifying crime patterns, making it easier to establish relations between crimes and other factors, such as urban infrastructure. Mirante has been evaluated by experts in public security, who gave us quite positive feedbacks, attesting the usefulness of our methodology.

Based on the modeling used in Mirante, in the next Chapter, we present CriPAV, a visual analytic methodology to scrutinize crime activities in a street-level of detail. In contrast to Mirante, CriPAV relies on mathematical and computational mechanisms to identify hotspot based not only on the intensity of crimes but also on their probability. 
CHAPTER

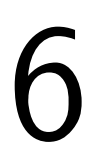

\section{CRIPAV: STREET-LEVEL CRIME PATTERNS ANALYSIS AND VISUALIZATION}

\subsection{Initial Considerations}

As mentioned in Chapter 4, Crime hotspot analysis has been one of the main resources employed by the public security agencies of most cities to plan police patrolling and design preventive actions (CHAINEY; TOMPSON; UHLIG, 2008). However, hotspot detection methods typically account for the absolute number of crime events in each specific location, neglecting sites where crimes are likely but do not occur in large numbers, mainly when compared to their surroundings, which can be more harmful to the community than intensive crime waves that occur in a short period of time (WANG et al., 2013a).

The issue above derives from the fact that there is no consensus about the definition of a hotspot, as such definition strongly depends, among other factors, on the spatial discretizations that support hotspot computation. The most common spatial discretization is a regular grid with cell granularity varying according to the scale of the analysis, which can range from dozen of meters to large areas covering entire neighborhoods.

However, crimes are mostly concentrated in 'micro' places that are relatively stable over time (GROFF; WEISBURD; YANG, 2010). Therefore, any fine grained crime analysis demands a street-level discretization difficult to be reached with regular grids (WEISBURD; MORRIS; GROFF, 2009), as density and arrangement of streets may vary considerably across the city.

Another important aspect related to hotspot analysis is related to the reasons that lead to the appearance of a hotspot in a given location. According to environmental criminology, the concentration and persistence of crimes in certain locations are not random, that is, they occur due to prevalent characteristics present in those locations (MCCULLOCH et al., 2005). Some studies demonstrate that crimes are related to demography, population, socioeconomic level and 
Figure 29 - The proposed street-level crime visualization methodology, CriPAV, comprises three main steps. Hotspot Identification: identifying hotspots based on crime intensity and crime probability. Finding Similar Hotspots: hotspot time series embedding (Hotspot2Vec), clustering, and projection into a visual space. Crime X Urban Infrastructure: finding the relation between urban infrastructure and crimes.

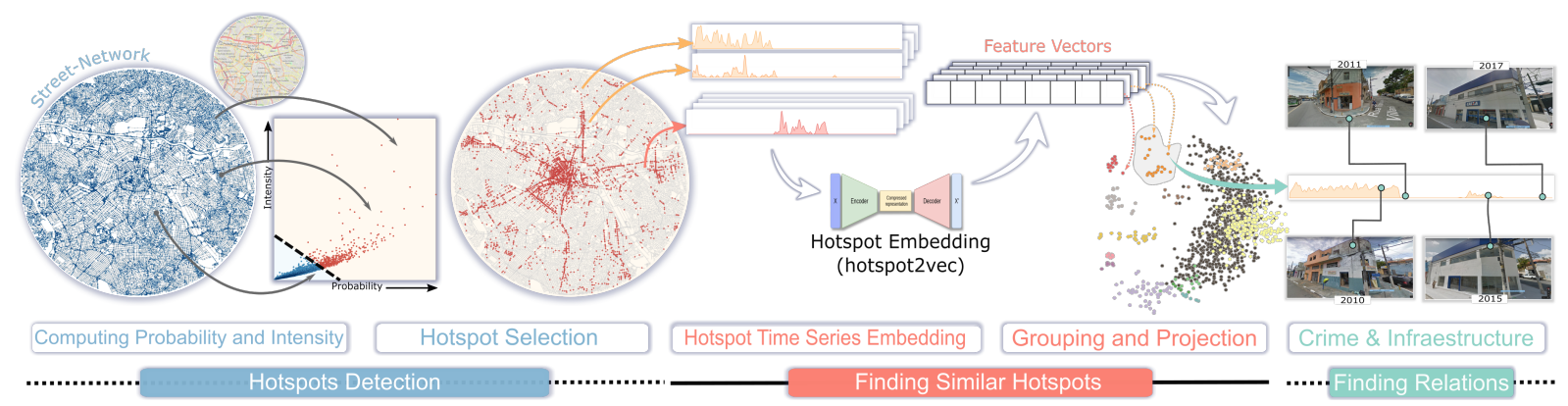

Source: Garcia-Zanabria et al. (2020b).

unemployment rates of each location (more details about environmental crime opportunities are presented in Section 2.4). Consequently, changes in the characteristics of particular locations impact crime activity over time, making the temporal analysis of hotspots a fundamental task (BRANTINGHAM; BRANTINGHAM, 1998; ECK; WEISBURD, 2015). Nevertheless, most hotspot based analytic tools, mainly the ones in use by security agencies, do not enable resources to identify and group hotspots according to their temporal behavior, hampering the identification of factors that can make crime viable or not.

In collaboration with domain experts and supported by mathematical and machine learning tools, we have designed a visual analytic methodology to scrutinize crime activities in a street-level of detail. Specifically, we use the same modeling of Mirante (presented in Chapter 5), which avoids the issue of finding a proper level of refinement to accomplish the analysis. The proposed methodology relies on mathematical and computational mechanisms to identify hotspot based not only on the intensity of crimes but also on the probability. Moreover, we rely on a deep learning model to embed crime time series in a high-dimensional space so as to make possible the identification of hotspot groups with similar temporal behavior, a task difficult to be performed with conventional hotspot analysis tools. The proposed methodology, illustrated in Figure 29, has been assembled in a visualization system called CriPAV, which, besides enabling a more general characterization of hotspots, provides visual resources to identify hotspot locations with similar temporal behavior. As shown in the provided use cases, the identification of hotspot locations with similar temporal crime behavior helps the understanding of how changes in urban infrastructure impact criminal activity over time. 


\subsection{Challenges}

The long term collaboration with a team of experts in crime analysis enabled us to raised a number of major challenges they face when performing their studies:

- Available hotspot analysis tools aggregate crimes in grid cells whose resolution covers entire neighborhoods. However, crime activity takes place in street-level sites within those neighborhoods, so detailed analysis demands a more fine-grain analytical mechanism.

- Hotspots are usually identified from the absolute number of crimes in the neighborhood of each grid cell. This approach typically neglect places where crimes are frequent but do not occur in large numbers. Frequent but not intense crime patterns (crime patterns account for the behavior of crime events over time) are quite relevant and deserve careful analysis.

- Their tools are only able to aggregate crimes spatially. However, crime patterns can change over time depending on the evolution of urban infrastructure, even in spatially close places. Such a temporal behavior can uncover interesting phenomena the experts can not observe with their regular tools.

These three challenges motivated a set of goals we aim to reach with the proposed methodology, which is presented in Section 6.2.1.

Figure 30 (right) shows an example of a region modeled as a network, where the black nodes are the anchor points.

\subsubsection{Goals and Analytical Tasks}

The set of challenges described above guided a set of goals to be reached. The first challenge culminated in the goal G1; the second in the goal G2; and the third in the goals G3, G4, and G5. Worth noticing that G5 helps in explaining the underlying reasons for an observed crime pattern.

- G1. Enabling fine-grained spatial analysis. There are many tools in which the unit of analysis is a region of the city (GARCIA-ZANABRIA et al., 2019; WILLIAMS; GALSTER; VERMA, 2014). However, inappropriate spatial aggregation might conceal the real behavior of crime patterns, leading to misleading conclusions. Our collaborators required an approach that enables analysis in a high level of detail, in particular, in the street-level of detail.

- G2. Accounting for the frequency and the absolute number of crimes to define hotspots. There is no consensus in the literature about a proper definition of what a hotspot should be. Therefore, authors tune the definition to suit their main purposes, most of them relying basically on the intensity of crimes to the hotspots. Our collaborators 
requested to consider not only the intensity but also how likely the location is to crime events. To reach that goal, we developed a new mathematical mechanism to measure the probability of a crime event take place in each anchor point. Domain experts emphasized this requirement because it is of great importance to improve public safety policy.

- G3. Inspecting the temporal behavior of crimes. In addition to the spatial analysis of crime events, it is also essential to understand the temporal behavior of crimes. Previous work highlighted the relevance of the temporal analysis for gaining a more comprehensive understanding of crime patterns (BRANTINGHAM; BRANTINGHAM, 1998; ECK; WEISBURD, 2015). Moreover, domain experts believe that many places, even in different locations, have similar temporal behavior. To validate this hypothesis, domain experts requested a tool to compare and explore crime patterns over time.

- G4. Grouping similar hotspots. Crime patterns can vary significantly, even in spatially close regions (GARCIA-ZANABRIA et al., 2019). However, spatially distant locations can present similar crime patterns due, maybe, to the resemblance of urban characteristics in those locations. Therefore, identifying locations with similar patterns is essential to understand which urban characteristics impacts in the type and pattern of crimes. Experts requested us for a technique that can automatically compare and group similar crime patterns.

- G5. Exploring and analyzing physical characteristics of particular locations. Understanding the impact of urban infrastructures such as public buildings, churches, banks, bars, or markets affect the type and pattern of crime, which is essential to drive security agencies public policy and decision-making. It is essential to explain, for example, "what it is more likely to occur" and "which type of crime is more prone to occur in a place with specific urban characteristics" (CAPLAN; KENNEDY, 2010). From domain experts' experience, neighborhood characteristics provide the context in which delinquent and crime events emerge. Visualization is critical to perform this analysis. For instance, geographical map representation with particular places is essential to mitigate exhaustive and time-consuming manual inspection. Given the importance of this analysis, the experts requested a tool to explore and analyze the relationship between urban infrastructure and crime patterns in specific places.

\subsubsection{Analytical Tasks}

After understanding the main goals of the project, we summarized the tasks our methodology/analytical tool must enable to address the raised requirements.

- T1. Probability $\times$ Intensity hotspots. Deliver to the expert the possibility of identifying hotspots based not only on the absolute number of crimes, but also on the probability of 
their occurrence. The tool should operate at a fine level of detail in order to enable fine grain analysis. This task helps on achieving requirements G1 and G2.

- T2. Geographical observation of hotspots. Depict the spatial distribution of crimes in order to facilitate the identification of regions with sparse and concentrated crime activities. This task helps on achieving requirements G1, G4, and G5.

- T3. Temporal analysis of crimes. Enable the temporal analysis of crime behavior in a particular hotspot or a group of hotspots. This task helps on achieving requirement G3.

- T4. Analysis of hotspot surroundings. Allow for experts to scrutinize the urban characteristics around a hotspot. This task helps on achieving requirement G5.

- T5. Grouping similar hotspots. Group hotspots with similar temporal behavior so as to facilitate the understanding of the reasons that lead to particular patterns. This task helps on achieving requirements $\mathbf{G 3}$ and $\mathbf{G 4}$.

- T6. Comparing hotspots' groups. Support the analysis of clusters by observing the inter/intra behavior of hotspots' groups, making possible to analyze the patterns of different groups and the dispersion of hotspots within a group. This task helps on achieving requirements $\mathbf{G 3}$ and $\mathbf{G 4}$.

Table 5 - Goals and their related analytical tasks.

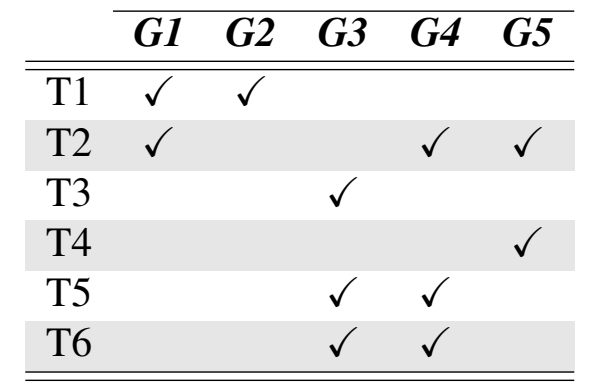

Source: Garcia-Zanabria et al. (2020b).

Table 5 summarizes of tasks achieving the experts' requirements. To integrate those tasks in a unified analytical tool, we have developed CriPAV, a system that enables a number of linked views each devoted to make doable one or more of the tasks above. The pipeline underlying CriPAV consists in three main components (see Figure 29): i) hotspots detection, ii) grouping hotspots with similar patterns, and finally, iii) interactive mining of patterns. In the following sections, we explain each of these components in detail.

\subsection{Data Modelling}

In this section, we describe the data we use in our analysis and case studies. First, we use the data set generated from São Paulo crime records (see Section 3.3.2). Then, aggregated the 
crime occurrences spatially in a street-level resolution, similar to data modeling used in Mirante (see Chapter 5).

Discussed and suggested by domain experts, we decided to take the streets intersection as the 'analysis unit' for practical and substantial reasons: $(i)$ due the availability of input data, urban features are unknown for us (FENG et al., 2020); (ii) When a crime event occurs generally, the police officer reports the location as the intersection of streets (BRAGA; HUREAU; PAPACHRISTOS, 2011); (iii) we can only measure the average of the criminality in a road segment, whilst the crime events positions are not exact (BRAGA; HUREAU; PAPACHRISTOS, 2011); (iv) Qualitative research suggests that robbers find street corners to be good locations to locate potential victims (JACOBS, 2000; JEAN, 2007); and (v) inner-city residents meet, socialize, and sometimes live out significant portions of their daily lives on streets corners (LIEBOW, 2003).

As mentioned previously, the spatial units of study are the anchor points. We model the region of interest as the street network where streets' intersections are the nodes (i.e., anchor points), and streets' segments are the edges. The spatial aggregation of crime events on the anchor points is accomplish in the same manner as in Mirante (see Algorithm 1 and Figure 30).

This data modeling helps to increase the resolution of crime analysis, thus leading to more refined and precise analysis.

Figure 30 - Data modeling process: (left) Region of study with anchor points (black dots) and criminal events (red dots), (center) approximation of each criminal event to the nearest anchor point, and (right) final street network, each anchor point contains a temporal series.

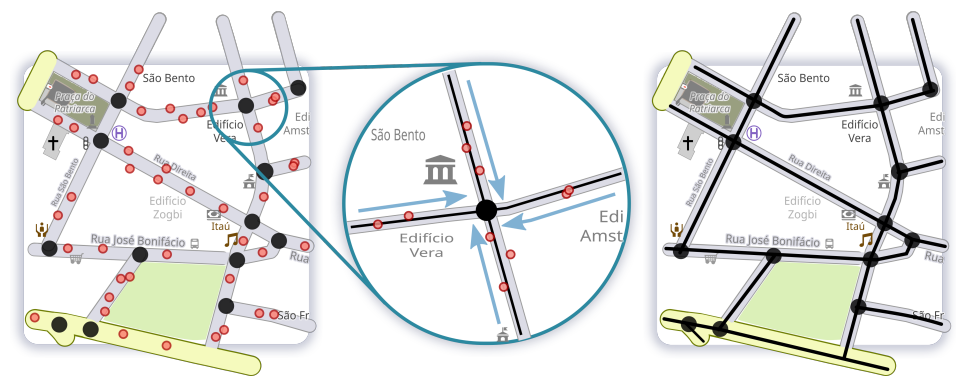

Source: Garcia-Zanabria et al. (2020b).

\subsection{Hotspots Detection}

One of the main components of CriPAV is the visual mechanism to select hotspots from the set of anchor points. The visual selection method builds upon a scatter plot of 'Probability $\times$ Intensity' of each anchor point, as illustrated in Figure 29 (Hotspot Detection). The intensity in a given location is the temporally aggregated number of crime occurrences in that location divide by the maximum number of occurrences among all locations. The computation of how likely crimes are in each anchor point is more intricate, and it will be detailed in the following sub sections. 


\subsubsection{Mathematical Foundation}

The probability of crimes take place in each particular anchor point is derived from the stationary state of a stochastic matrix built from the temporal crime data. Before describing the computation itself, we provide some important mathematical concepts.

Probability vector. In mathematics and statistics, a distribution vector $p$ is called a probability vector if all its elements are non-negative real numbers which add up to 1 (i.e., $\sum_{i=1}^{n} p_{i}=1$ ).

Stochastic Matrix. An $n \times n$ square matrix $P=\left(p_{i j}\right)$ is said stochastic if each row (or column) is a probability vector. Stochastic matrices are used to describe the transitions of a Markov chain (SZABO, 2015), that is, each entry $p_{i j}$ is the probability of changing from state $i$ to state $j$.

Under certain mild conditions, it can be shown that a stochastic matrix has at least one eigenvalue equal to 1 . The eigenvector associated to the eigenvalue 1 is the stationary vector of the stochastic matrix $P$, and it corresponds to a probability vector that is the solution of the following optimization problem:

$$
\arg \min _{\pi}\left\|\pi^{\top} P-\pi\right\|^{2} \quad \text { subject to } 0 \leq \pi \leq 1
$$

In our context, the probability of crimes in each anchor point is given by the stationary vector of a stochastic matrix built from the crime time series in each anchor point. The construction of such stationary matrix is detailed in the following.

\subsubsection{Computing the Stochastic Matrix}

We need to compute the stochastic matrix from which the probability of crime in each anchor point is derived.

Given a street network with a set of $n$ anchor points $V=\left\{\tau_{1}, \tau_{2}, \ldots, \tau_{n}\right\}$ and a set of $m$ time units $T=\left\{t_{1}, t_{2}, \ldots, t_{m}\right\}$, the crime time series in each anchor point can be seen as a function $f: V \times T \rightarrow \mathbb{R}$ that associates the number of crime events $f\left(\tau_{i}, t_{j}\right)$ in each anchor point $\tau_{i}$ in each time unit $t_{j}$. We denote by $D$ the $n \times m$ matrix which contains all the values $f\left(\tau_{i}, t_{j}\right)$, thus every entry $D_{i j}$ in $D$ are greater or equal to zero. We define an occurrence matrix $\widehat{D}$ where $\widehat{D}_{i j}=1$ if $f\left(\tau_{i}, t_{j}\right)>0$ and $\widehat{D}_{i j}=0$ if $f\left(\tau_{i}, t_{j}\right)=0 . \widehat{D}$ is a binary matrix where each entry $\widehat{D}_{i j}$ indicates whether crimes took place in the anchor point $\tau_{i}$ and time slice $t_{j}$.

We denote by $\widehat{P}$ the $n \times n$ co-occurrence matrix defined as:

$$
\widehat{P}=\widehat{D} \cdot \widehat{D}^{T}
$$

Each entry $\hat{p}_{i j}$ provides the number of times that the anchor points $\tau_{i}$ and $\tau_{j}$ present crime events in the same time unity, that is, the number of times that crimes took place simultaneously in $\tau_{i}$ and $\tau_{j}$. A large $\hat{p}_{i j}$ value means that $\tau_{i}$ and $\tau_{j}$ present similar crime activity. Dividing each row of $\widehat{P}$ by the sum of its values we end up with an stochastic matrix $P$, that is, $p_{i j}=\hat{p}_{i j} / \sum_{k=1}^{n} \hat{p}_{i k}$. 
Figure 31 - (a) Probability versus Intensity scatter plot, (b) filtering based on intensity and probability with $\alpha=1$, and (c) filtering based on intensity and probability with $\alpha=0.3$, the red anchor points are the hotspots. Parameter $\alpha$ controls the slope of the separation line. The value of $\alpha$ is the weight one wants to give to intensity of probability when filtering the hotspots.

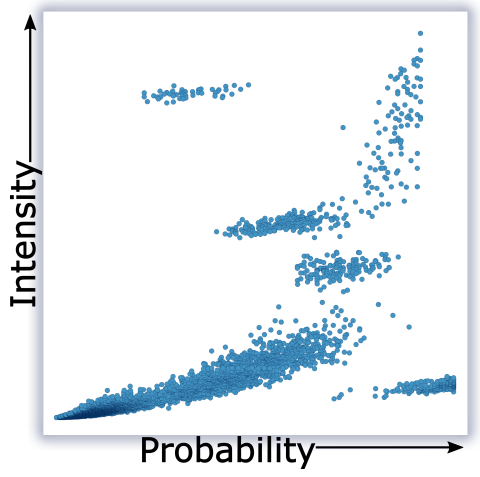

(a) P. \& I.

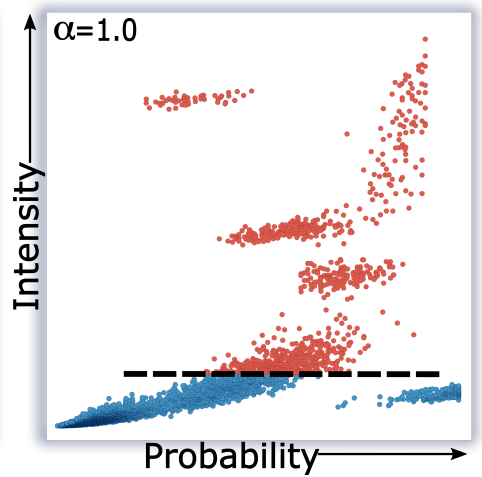

(b) Filtered P. \& I. with $\alpha=1$

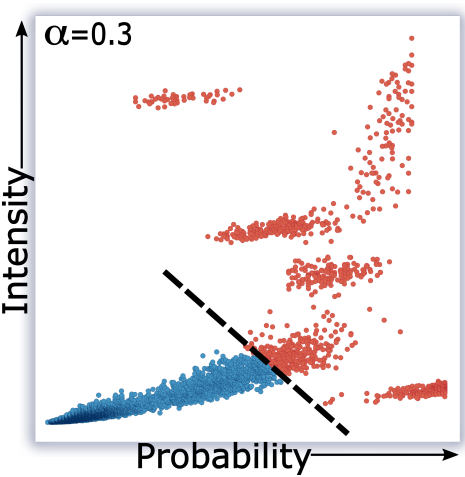

(c) Filtered P. \& I. with $\alpha=0.3$

Source: Garcia-Zanabria et al. (2020b).

The entry $p_{i j}$ corresponds to the probability $\operatorname{Pr}\left(\tau_{i}, \tau_{j}\right)$ of a crimes take place simultaneously in $\tau_{i}$ and $\tau_{j}$. The reasoning behind the construction of the stochastic matrix $P$ is that certain crime types are seasonal, occurring concurrently in different city locations depending on the day of the week, the fortnight of the month, and the month of the year. Matrix $P$, as defined above, captures such a seasonality, being able to point as likely an anchor point where crime activity is not intense, but occur concurrently with other anchor points.

Given the stochastic matrix $P$ the probability of crime occurrence in each anchor point is given by the stationary vector $\pi$ of $P$, that is, the probability of a crime event occur in $\tau_{i}$ is the value in the $i$-th entry in $\pi$.

\subsubsection{Selecting Hotspots}

The probability and intensity values summarizing crime activities in each anchor point enables the use of a Probability vs. Intensity scatter plot to visually identify high intensity anchor points (the top ones in the plot), anchor points with high probability (the right most ones in the plot), and anchor points of high probability and intensity, as illustrated in Figure 31 (a).

In order to filter out relevant anchor points (i.e., high probability and/or high intensity), use a function $g=[0,1] \times[0,1] \rightarrow[0,1]$ that assigns a value to each anchor point, as for example $g($ probability, intensity $)=((1-\alpha) *$ probability $+\alpha *$ intensity $)$. The value of $\alpha$ is the weight one wants to give to intensity and probability when filtering the hotspots. Parameter $\alpha$ controls the slope of the separation line. However, we need to position the line in the plane. To do this, we order each anchor point based on the value returned by the function $g$, displacing the line based on the percentage of anchor points that should be considered as hotspots. In Figure 31 (b, c), we show the plots with $\alpha=1$ and $\alpha=0.3$ respectively. The dotted line represents the function, and 
the red points are the 5\% most relevant anchor points, which correspond to the hotspots.

We have implemented the linear hotspot selection mechanism as an alternative to the interactive brush-based interactive tool. Domains experts deemed the linear approach easier to use than a brush-based one.

Figure 32 - (1) Region of interest. (2) time series containing information of interest anchor points in (1). (3) filtering in Probability versus Intensity. (4) Hotspots clusterization and LAMP visualization based on hotspot2vec.

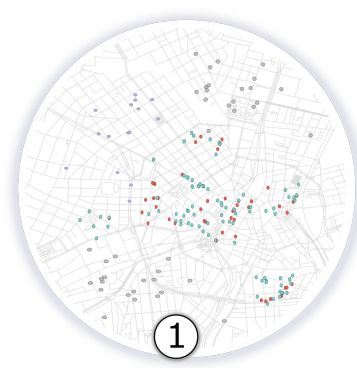

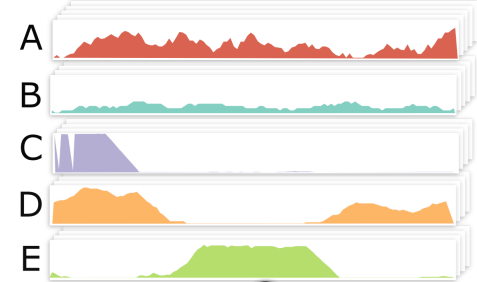

(2)
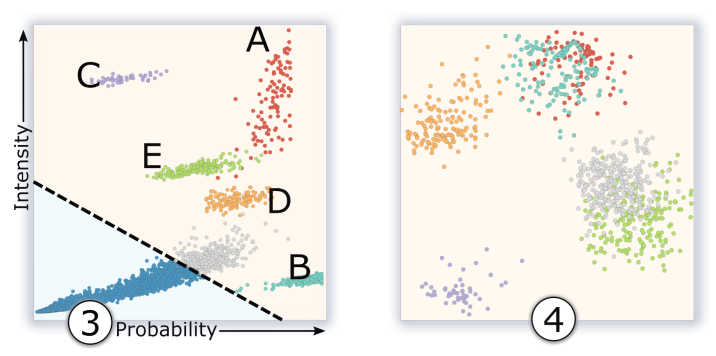

Source: Garcia-Zanabria et al. (2020b).

\subsubsection{Validation - Selecting Hotspots}

To evaluate the hotspot selection approach, we created a synthetic data set with 14,000 anchor points and 120 time-slices representing months over ten years. Figure 32 (1) shows the region of study where anchor points are labeled based on five situations: high intensity $\&$ high probability (A - crimes occurring all over the time), low intensity \& high probability (B - small number of crimes occurring in most time units), high intensity \& low probability ( $\mathrm{C}$ - a large number of crimes concentrated in some time units), crimes that only occurs in the beginning and at the end of the time interval (D), and crimes occurring only in the middle of the time interval (E). The anchor points with low intensity and low probability are not of interest. Figure 32 (2) shows some time series from each category.

Given the time series on each anchor point, we calculate the probability and intensity using the methodology described above. Figure 32 (3) illustrates the probability vs. intensity plot, where points on the right of the dotted line correspond to the $5 \%$ most relevant anchor points. We use $\alpha=0.3$ as the cutting function. Notice that points on top-right correspond to group A (red points), presenting high probability and intensity values. Bottom-right points correspond to group $\mathrm{B}$, that is, anchor points with frequent but not intense crime rates when compared to $\mathrm{A}$ and C. Finally, top-left points correspond to group C, where crimes are not frequent (low probability) but have a high intensity in a certain periods of time. The remaining points (orange and light green) show a moderate level of probability and intensity. Worth mentioning that by pushing the decision towards intensity ( $\alpha=1$ makes the line horizontal), we miss group $\mathrm{B}$, which is important since it has criminal activity all over time. Favoring intense hotspots is what is done in the most existing hotspot analysis tool. 


\subsection{Finding Similar Hotspots}

Another important task that must be accomplished by our methodology is the identification of hotspots with similar temporal behavior. Finding temporally similar hotspot means to search for similar time series, which is known to be a difficult problem. Methods such as Discrete Time Warping (DTW) can be used to this end, but with the price a high computational cost and instability to noise (GOLD; SHARIR, 2018). Instead, we opt for a deep learning embedding technique that we called Hotspot2Vec.

Hotspot2Vec. We use an autoencoder to map each time-series $T S=\left\{t s_{1}, t s_{2}, \ldots, t s_{m}\right\}$ to a feature space. The autoencoder model is trained with a set of time-series $\widehat{T S}=\left\{\widehat{t s}_{1}, \widehat{t s}_{2}, \ldots, \widehat{t s}_{m}\right\}$, where $\widehat{t s_{i}}=1$ if $t s_{i}>0$ and $\widehat{t s}_{i}=0$ otherwise, for all $i \in\{1, \ldots, m\}$. The idea is to train the deep learning model to capture the temporal behavior of crimes, without taking into account the intensity of crimes. Therefore, anchor points with crimes happening at the same time interval will be considered similar, no matter the intensity of crimes in each location. Autoencoder is a well-known neural network model in which the input and output are the same, and the middle layer of the network has a bottleneck that creates a compressed representation, aiming at reducing the dimensionality of the data.

The autoencoder architecture consists in an encoder with 3 pairs of $1 \mathrm{D}$ convolution and max-pooling layers. The first two convolution layer have 16 kernels of size 3 , and the last convolution has a filter of size 3 . In our

Figure 33 - Autoencoder result: the blue line represent the original time series, and the orange ones are the decoded time series.

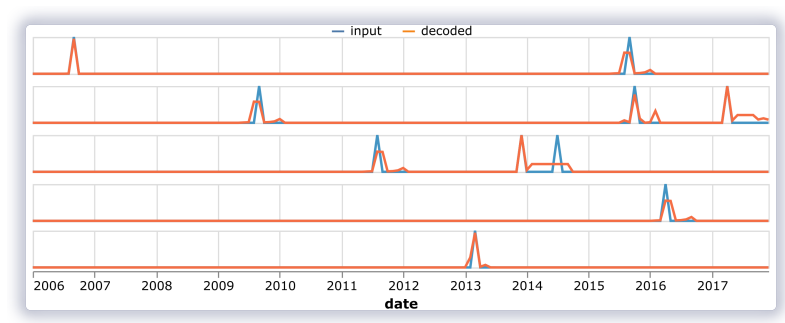

Source: Elaborated by the author. case, the time series contains 144 values, and in each convolution, the time series is reduced by half, reaching a size of 18 . It is important to mention that we use convolutional layers to capture local information around each time step. In this way, we can match two time-series that have small shifts.

Our decoder has a similar architecture to the encoder but changing the max-pooling layers to up-sampling layers. All the convolution layers use Relu activation function except for the last one, which uses a Sigmoid function. Figure 33 depicts the result of autoencoder in reconstructing the time series.

Grouping Similar Hotspots. The output of the encoder is used as feature vectors, and a clustering algorithm is applied to group hotspots based on their proximity in the feature space. We choose a hierarchical variant of Density-Based Spatial Clustering (DBSCAN) (ESTER et al., 1996) called Hierarchical Density-Based Spatial Clustering (HDBSCAN) (CAMPELLO et al., 2015). The choice for HDBSCAN is because it is capable of automatically finding the number of 
clusters (as DBSCAN) without tuning several parameters, thus relieving users of this task, what is essential for the crime analysis domain experts that have little training in machine learning.

Projection. Empirical tests showed that reducing the dimensionality of time series to 18 still preserve good properties in terms of capturing their similarity. To visualize the resulting embedding, we relied on a modified version of the Local Affine Multidimensional Projection $\left(\right.$ LAMP $^{1}{ }^{1}$ technique (JOIA et al., 2011), which maps the embedded time series to a 2D visual space. LAMP is a computationally efficient projection method that can be tuned to preserve labeled clusters during the mapping. Figure 29 (Finding Similar Hotspots) shows an example of the HDBSCAN clusterization and LAMP projection.

\subsubsection{Validation - Grouping Similar Hotspots}

We use the same synthetic data set created for validating the intensity $\times$ probability hotspot selection. After selecting the hotspots using the scatter plot, we applied Hotspot2Vec and HDBSCAN to estimate the groups. Figure 32 (4) shows the 2D projection resulting from LAMP. Notice that three time series types $(\mathrm{C}, \mathrm{D}$, and $\mathrm{E}$ ) out of the five original ones were properly clustered, while groups A and B were, as expected projected on the top of each other, since the embedding doesn't account for the intensity of crimes, so they are considered similar.

\subsection{Visual Components}

We designed a set of visual components to integrate the hotspot detection and grouping described above. This system, depicted in Figure 34, provides six components: (a) a map view to visualize anchor and hotspots; (b) a scatterplot showing the distribution of anchor points according to intensity and probability of crimes in each anchor point; (c) a projection-based view of hotspots in a two-dimensional space; (d) a visual representation of hotspot groups; (e) visualization of time series in a particular group; and (f) a photo panel showing urban infrastructure over time for a selected anchor point. We design these visual components in close collaboration with domain experts and their requirements. Each view has been designed to address at least one analytical tasks described in Section 6.2.2. Table 6 indicates the relation between the visual resources and tasks(columns). For instance, Within-Group Joy Chart and Between-Group Chart account for three tasks. We detail each visual component in the following sections.

$1<$ github.com/lgnonato/LAMP> 
Figure 34 - CriPAV system: Hotspot, spatial, and temporal interactive views enabling the exploration of local regions while revealing their criminal patterns over time.

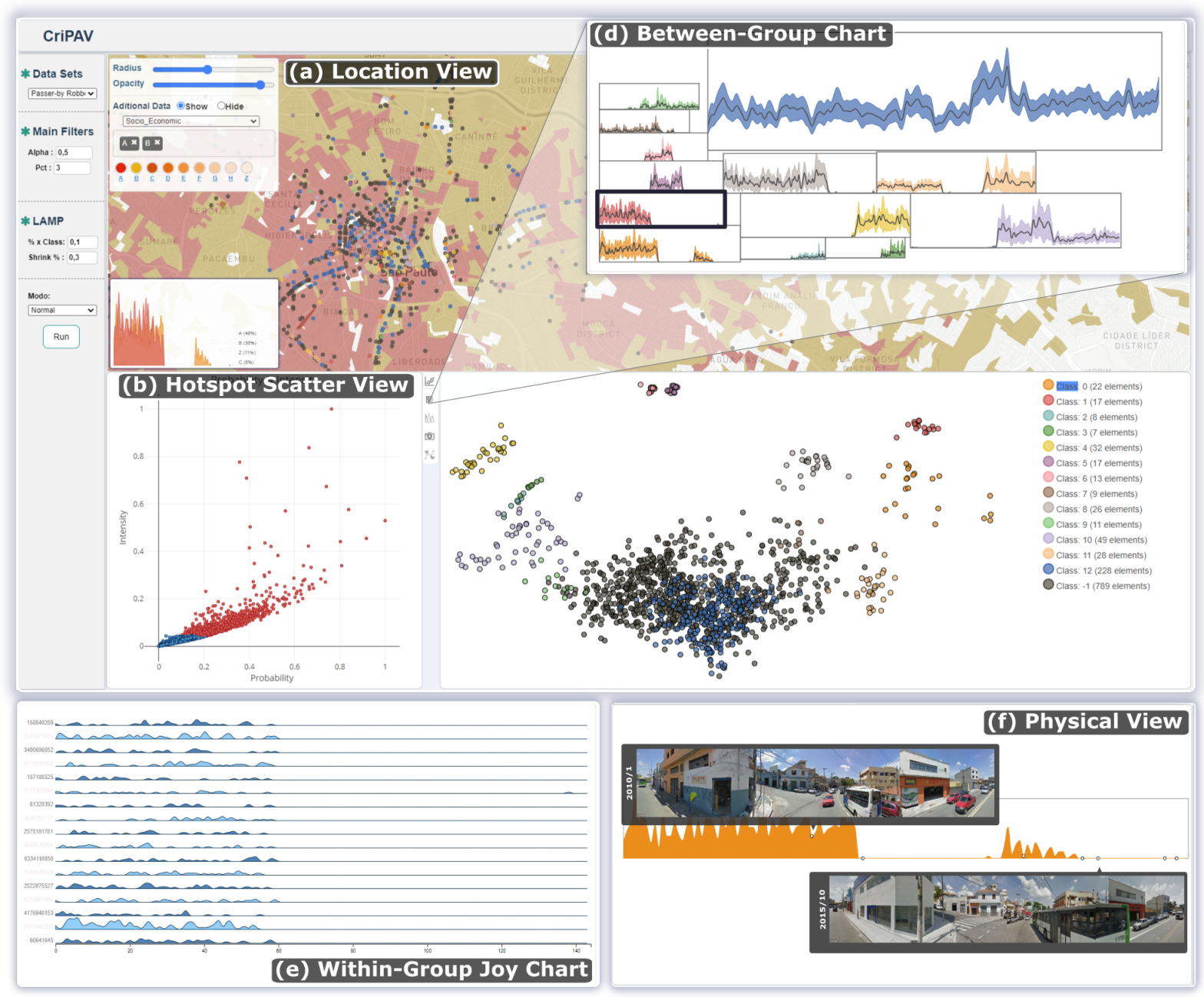

Source: Garcia-Zanabria et al. (2020b).

Table 6 - Methodological and visualization properties and their related tools.

\begin{tabular}{cccccccc} 
& Sec. & T1 & T2 & T3 & T4 & T5 & T6 \\
\hline \hline Location view & Sec. 6.6.1 & & $\checkmark$ & & & & \\
Hotspot scat. view & Sec. 6.6.2 & $\checkmark$ & & & & & \\
Temporal Patt. Ploj. & Sec. 6.6.3 & & & $\checkmark$ & & $\checkmark$ \\
B-Group chart & Sec. 6.6 .5 & & & & $\checkmark$ & $\checkmark$ & $\checkmark$ \\
W-G Joy Chart & Sec. 6.6 .4 & & & & $\checkmark$ & $\checkmark$ & $\checkmark$ \\
Physical view & Sec. 6.6.6 & & $\checkmark$ & & & \\
\hline \hline
\end{tabular}

Source: Garcia-Zanabria et al. (2020b).

\subsubsection{Location view}

This view, depicted in Figure 34 (a), enables the visualization of the geographical location of the anchor points. The color of each node depends on the group it belongs to, which 
is computed as described in Section 6.5. The location view is particularly useful to overview the spatial distribution of hotspots and their similarity. As shown in Figure 34 (a), location view provides $2 \mathrm{D}$ and $3 \mathrm{D}$ visualization of the hotspots, being possible to change visualization properties such as size, opacity, and elevation (in the 3D option) of the hotspots. Color opacity and elevation, the latter only available in the $3 \mathrm{D}$ view, can be set to correspond to the intensity or probability of the hotspots.

Anchor Point Selection. By simple clicking in each anchor point, Physical View shows the Google Street View photos of the selected hotspots.

\subsubsection{Hotspot Scatter View (Probability vs Intensity)}

Shown in Figure 34 (b), the hotspot scatter view show Probability\&Intensity scatter plot of the hotspots. To identify anchor points as hotspots, one can use the linear discriminant filter function described in Section 6.4 by tuning the parameters $\alpha$ and pct, although an interactive brushing mechanism is also available.

\subsubsection{Crime Pattern Projection}

Once hotspots have been selected they are grouped according to similarity and a multidimensional projection is performed to reveal their distribution in the feature space. As shown in Figure 34 (c), hotspots are colored according to their groups in the projection. Moreover, the legend on the right encodes the label of each group and the number of elements in each group.

Group Selection. By clicking in the label of each group, Location View, Between-Group Chart, and Within-Group Joy Chart are updated to highlight only the data in the selected group, making it easier for users to focus their analysis on a specific group of hotspots.

Filtering. In this view, it is possible to select hotspots using D3js-lasso selection. The filtered hotspots are highlighted in the Location View and Hotspot Scatter View. In addition, one can analyze individual time series of selected hotspots using the Within-Group Joy Chart.

\subsubsection{Within-Group Joy Chart}

This view relies on filled lines (see Figure 34 (e)). Each line chart encodes the number of crimes in the hotspots of a specific group over time. This visualization helps in understanding the crime pattern of the hotspots in a group.

Time Series Selection. By clicking in each area chart, Physical View shows the photos of the surroundings of the selected hotspot. 


\subsubsection{Between-Group Chart}

To complement the intra-cluster representation of the Within-Group Joy Chart, we also create a visual representation summarizing the crime pattern in a group (see Figure 34 (d)). This visualization consists in showing only one time-series of each group with glyph size proportional to the number of hotspots in that group. These rectangular glyphs are arranged so as to keep the most similar groups closer to each other in the layout, following the proximity between groups depicted in the Crime Pattern Projection view, optimizing the view is used as much as possible. The optimization is a variant of the method described in (GOMEZ-NIETO et al., 2015), and it is detailed in the supplementary material. This visualization is useful to understand the relation and difference among the crime patterns present in the data.

Group Selection. By clicking in the rectangle of each group, Crime Pattern Projection, and Within-Group Joy Chart are updated to highlight only the data of that group.

Time Series Selection. By clicking in each area chart, Physical View shows the photos of the surroundings of the selected hotspot.

\subsubsection{Physical View}

This view uses google street view to extract and organize temporally photos of the surroundings of selected hotspots (see Figure 34 (f)). Each photo is a collage of many (at least four) photos extracted during spatial padding. This padding is accomplished for each time slice. Physical View helps domain experts to understand the relation between crime patterns and the change in urban infrastructure over time.

\subsection{Implementation}

CriPAV is a web-based system under the python Flask server. The core of the system is divided into data/space modeling, computation of hotspots, and visualization modules. For the data/space modeling we used OSMnx ${ }^{2}$ (BOEING, 2017) and NetworkX ${ }^{3}$ python libraries to extract street network and crime events aggregation respectively. To achieve interactive rates, we pre-processed the data (i.e., computation of the nearest edges, vertex nodes, and street network simplification). For hotspot identification (i.e., computation of stochastic matrix, node probability, and intensity), we used Pandas and Numpy python libraries. Finally, all visualization resources have been developed based on JavaScript library: Deck-GL ${ }^{4}$, to perform the geomap representations (i.e., scatter-plot and choropleth map), Plotly $\mathrm{Js}^{5}$ for Probability $\times$ Intensity

\footnotetext{
$<$ https://osmnx.readthedocs.io/>

$<$ https://networkx.github.io/>

$<\mathrm{https}: / /$ deck.gl/>

$<$ https://plotly.com/javascript/>
} 
representation, D3.js ${ }^{6}$ to represent scatter-plot, line, and area charts. In our case, we have developed an extra component to manipulate Google Street View photos based on StreetView ${ }^{7}$ to SKImage (WALT et al., 2014) python libraries.

\subsection{Case Studies}

In this section, we present three case studies applying our methodology on a real crime data set from São Paulo - Brazil. These case studies show different analytical strategies while addressing analytical tasks described in Section 6.2.2. The first case study addresses T1, T2, and T5, highlighting the importance of accounting for probability in hotspot detection. The second case study focuses on the relation between hotspots and socioeconomic factors, addressing $\mathrm{T} 2$ and T4. The third case study aims to show the potential of CriPAV to assist the experts in their search for possible explanations for crime patterns, relating similar patterns to urban infrastructure (T3 and T6). In all case studies, except when explicitly stated, we focus on passerby robbery as the crime type under analysis.

\subsubsection{Intensity only versus Intensity \& Probability}

The main goal is to use the proposed framework to analyze the impact of selecting hotspots based only on crime intensity versus select them from the Probability vs. Intensity plot.

Figure 35 shows the Hotspot Scatter View (top-left graphic) where red dots are anchor points selected as hotspots in both the Intensity only and Intensity \& Probability. The blue dots are anchors considered in the Intensity \& Probability only, while the green dots are anchors considered in the Intensity only group. The gray dots are not considered hotspots. The WithinGroup Joy Charts (top-right line plots) show crime patterns from the blue and green set of hotspots. The Location View (bottom map) shows the distribution of blue and green hotspots in a specific region of the city.

It is possible to notice that the set of green hotspots left out in the Probability x Intensity selection correspond to crimes that take place in a short period of time, while the probable hotspots (blue dots) left out in the Intensity only selection contain patterns of crimes spread out over the whole period of time An interesting aspect pointed out in the Location View (Figure 35 (bottom)) is that the green (probable) hotspots tend to show up along main streets, avenues, and highways (dashed lines) while the blue (intense) hotspots are more spread, appearing also in secondary streets. Domain experts considered this an interesting finding because it shows that crime patterns seem to change according to urban infrastructure in a way they have not observed before. The possibility of identifying those patterns can make public security policies more efficient.

$6<$ https://d3js.org/>

$7<$ https://github.com/robolyst/streetview> 
Figure 35 - Analysis of hotspot identification technique. Comparison between missed hotspots with different cut variables. (left) Probability versus Intensity, red dots are hotspots identified by Intensity and Mixed definitions, blue dots are hotspots identified just by Mixed definition, and green dots are points identified only by Intensity Definition. (middle) The time series of blue and green dots. (right) Location View with plotted points.

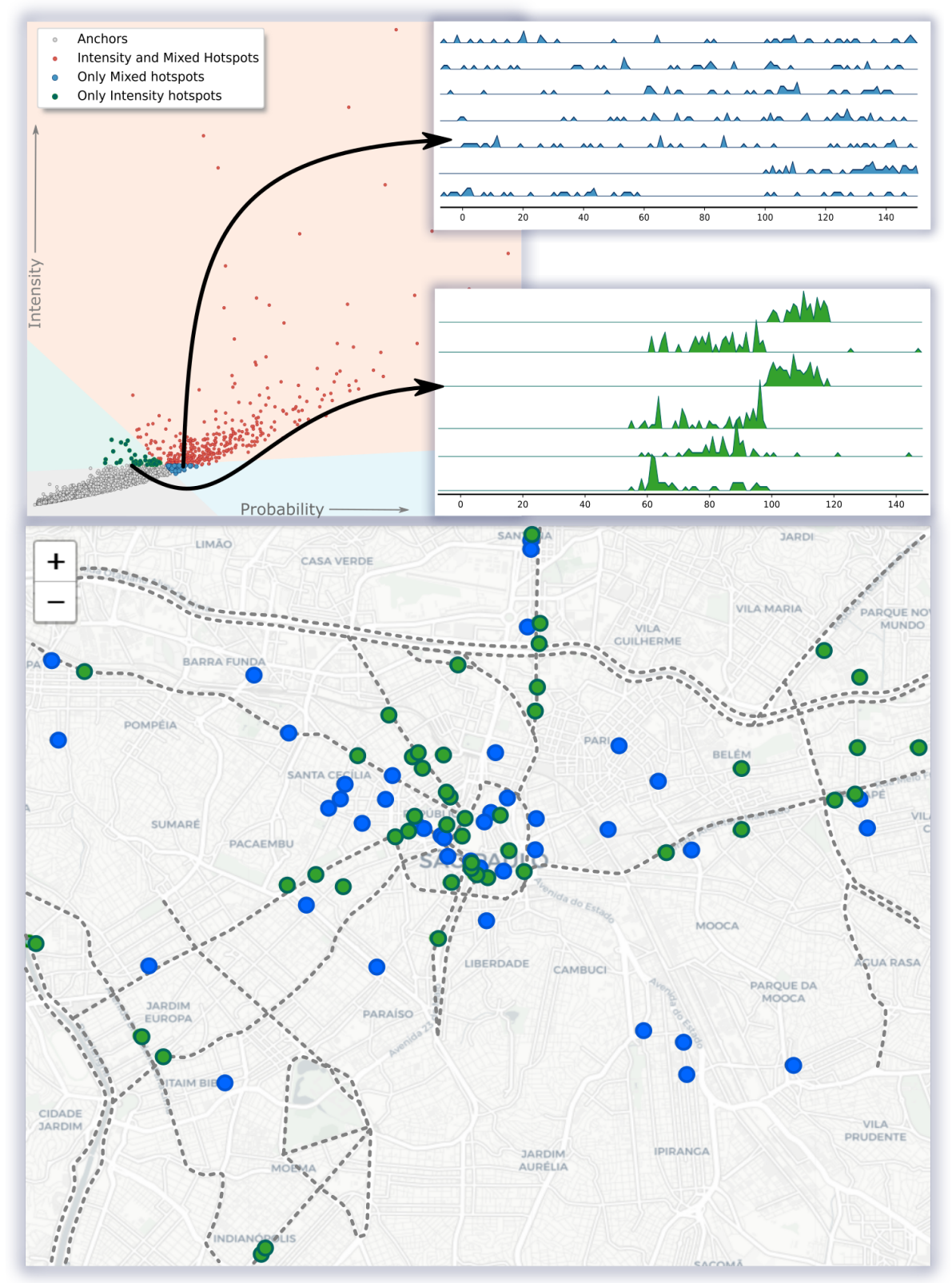

Source: Garcia-Zanabria et al. (2020b).

CriPAV enables the selection of both types of hotspots, and more importantly, it is capable of showing the importance of using the probability to characterize hotspots. Therefore, the case study shows that the proposed methodology is able to found robust hotspots based not only on the Intensity but also on the Probability (T1) while showing the geographical localization of the hotspots (T2). Finally, it is possible to analyze different crime patterns (T5) and their relation with urban characteristics. 


\subsubsection{Understanding Crime Patterns Over the City}

The main goal is this case study is to analyze spatial and temporal crime patterns over the city, relating those patterns with the socioeconomic properties of each location.

Specifically, domain experts aimed to address the following questions: (i) how are hotspots concentrated for each type of crime?; (ii) how are crime patterns spatially distributed?; and (iii) is there any correlation between hotspots and socioeconomic variables?. Domain experts provided socioeconomic indicators that account for population, housing, mobility, and urban expansion in each census unit, labeling those units according to eight categories ranging from A (better socioeconomic level) to $\mathrm{H}$ (worst level). We selected a region of study with 5,890 census units spread around downtown São Paulo. First, the location view was used to visualize the concentration of hotspots. Figure 36a shows the hotspot distribution for each crime type. The colored polygons in the background correspond to census units of level A (red) and B (orange). Notice that there are three prominent patterns of hotspot distribution. Passerby robbery hotspots are concentrated in the center of the city, Commercial Establishment robbery is scattered throughout the city, while Vehicle robbery hotspots are concentrated in the periphery. The socioeconomic indicators are also related to the crime types. The bottom legend in Figure 36a shows the percentage of hotspots in each socio-demographic region. We can see that Passerby robbery is more prevalent in class A, Commercial Establishment robbery is equally distributed, and Vehicle robbery takes place mostly in class B. Moreover, the passerby, commercial establishment, and Vehicle robberies hotspots are concentrated in only a small portion of the census unit, $535(9 \%), 305(5 \%)$, and 591 (10\%), respectively.

The relation between socioeconomic factors and crime types is even more pronounced when we take into account the temporal behavior. During the exploration of the three crime types using the CriPAV tool, domain experts perceived four prominent temporal patterns. Relying on Between-Group Chart, it was possible the notice the patterns depicted in Figure 36b left, where the four patterns are clearly seen, namely, high criminality in the past, high criminality in the recent past, recent high criminality, and criminality all over the time. Socioeconomic factors seem not to affect passerby robbery in any pattern. This might happen because this type of crime is highly related to people's flow; it doesn't matter if in class A or B. In contrast, socioeconomic factors have a great influence on crime patterns regarding Commercial Establishment and Vehicle robberies. It seems that lower class neighborhoods have become more vulnerable to those types of crimes over time. In the past, both types of crime were nearly equally distributed between socioeconomic class $\mathrm{A}$ and $\mathrm{B}$, However, in more recent years, we notice a shift in the percentage of crimes from class A to class B. The reason for that shift is an interesting aspect to be further investigated, and the expert has never noticed that phenomenon before. 
Figure 36 - Comparison of Passerby, Commercial Establishment, and Vehicle robberies patterns over the city. (a-first row) Concentration and dispersion tendency of hotspots; (a-second row) Relation between hotspots and socioeconomic variables, and (b) Spatial behavior of hotspots clusters and socioeconomic variables.

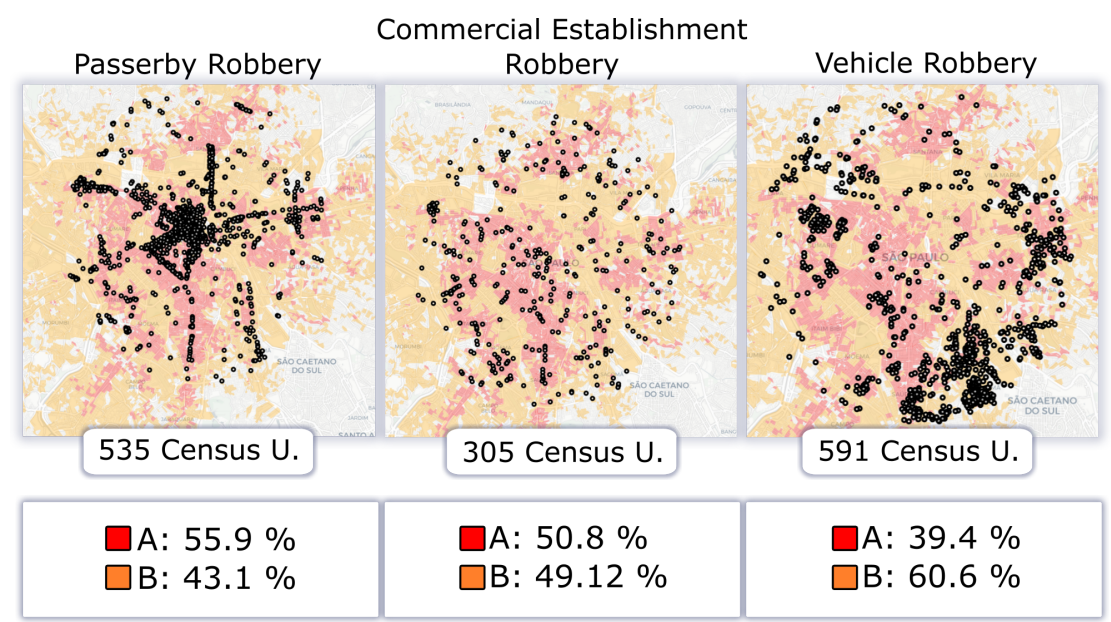

(a) First row: Location View of hotspots distribution of each crime type and number of census units involved. Second row: the relation between hotspots and socioeconomic variables for each crime type.

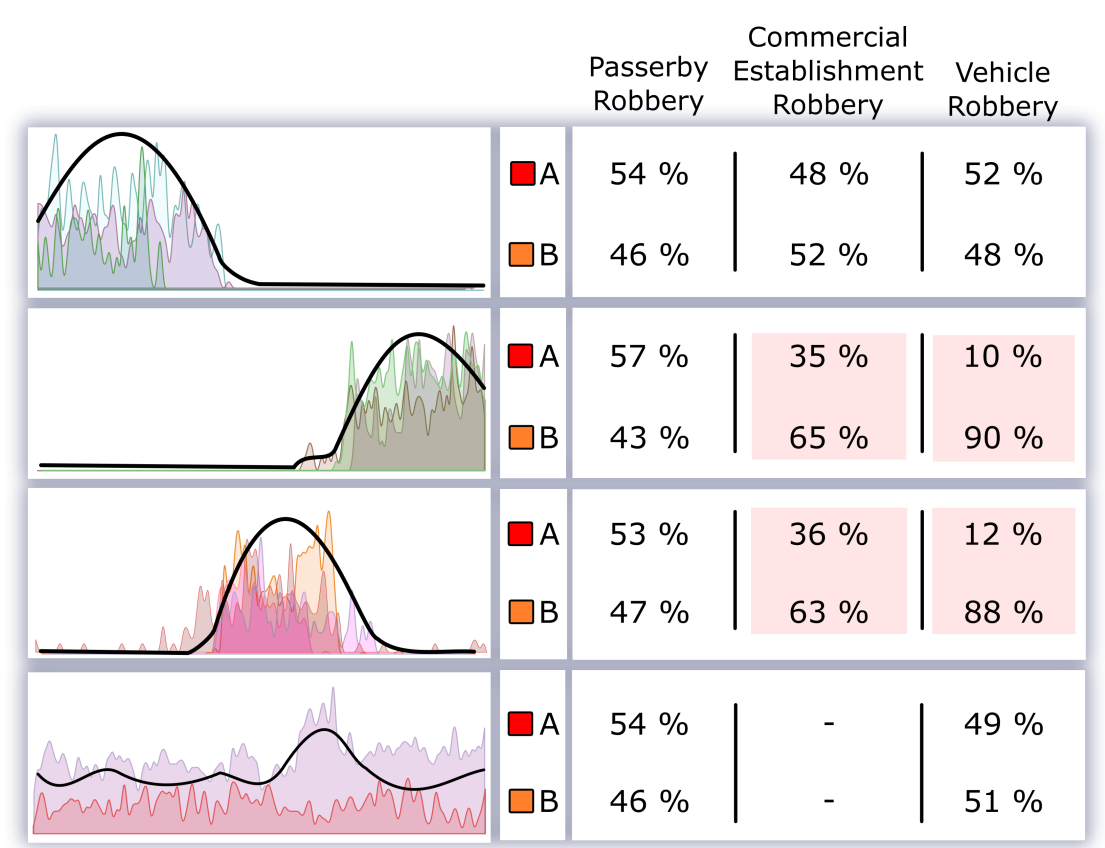

(b) Relation between Between-Group Chart temporal patterns and socioeconomic variables. First row, the four most prominent temporal patterns. Second row, the most prevalent socio-economic classes $(\mathrm{C}-\mathrm{H}$ are not considered because the sparcity). Third row, percentage of hotspots of a temporal pattern that are located in each socio-economic class.

Source: Garcia-Zanabria et al. (2020b).

The discussion above shows that the hotspot detection and the grouping of similar hotspots analytic functionalities implemented in CriPAV are able to sort out the three analytical questions raised by domain experts. In particular, Location View and Between-Group Chart turn out to be effective in showing the spatial distribution of the hotspots (T2) and the relation between hotspots and the characteristic of their surroundings (T4). 
Figure 37 - Hotspots infrastructure comparisons over time. (1) Clusterization and projection of hotspots using Hotspot2Vec, (2) Between-Group Chart showing temporal behavior of each group, (3) time series of the selected group of hotspots (Group 0 and Group 2), and (4) temporal images of point $\mathrm{P} 1, \mathrm{P} 2$, and $\mathrm{P} 3$.

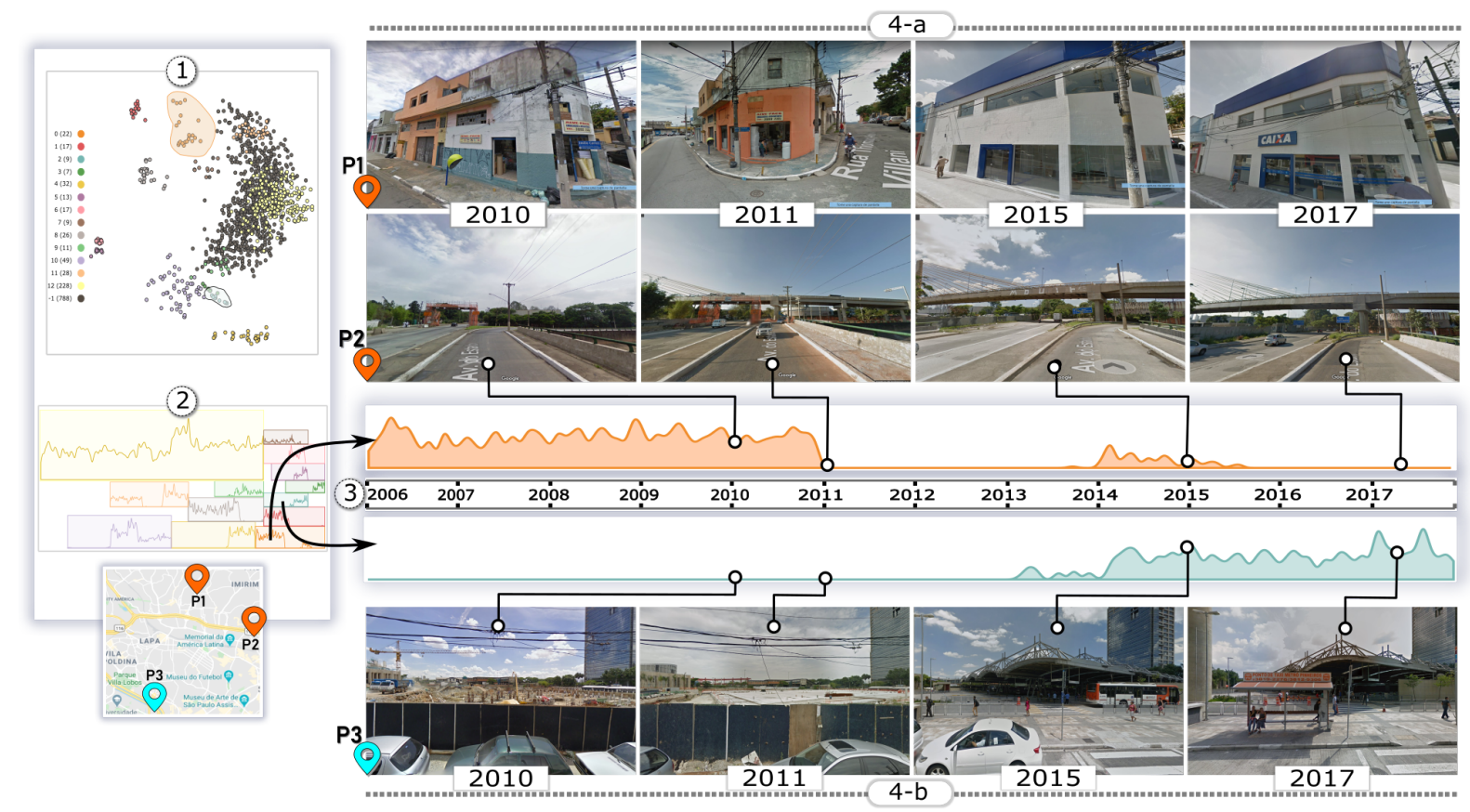

Source: Garcia-Zanabria et al. (2020b).

\subsubsection{Relating Urban Infrastructure and Crime Patterns}

This case study assesses the effectiveness of CriPAV in assisting experts to analyze the relation between crime patterns and urban infrastructure. To this end, it is necessary to explore crimes in a fine grain level of detail (T2), analyze the physical characteristics of each location (T3), and comparing different hotspots groups (T6).

We used Hotspot Scatter View to select 3 percent of the most important hotspots considering both probability and intensity. Figure 37 (1) shows the groups and projection of the selected hotspots. The color encodes the different groups, and the legend on the left represents the id and the number of elements in each group.

The Between-Group Chart (Figure 37 (2)) presents a visualization of the groups and their summarizing time series. To perform a detailed analysis, we selected three hotspots denoted as P1, P2, and P3 in the map shown on the left bottom of Figure 37. P1 is in the north zone of the city, in an industrial area. P2 is located in the east part of the city, and it is surrounded by recreational places such as sports clubs and Samba schools. P3 is located in a district that has high quality infrastructure in terms of transport, health, culture, and education, and it is surrounded by a metro station, a university, and a bus terminal. Figure 37 (3) top row shows the time series of group 0 and group 2. These time series were extracted using the Between-Group Chart. Notice that time series of group 0 (orange one), present relatively high criminality rates in the early years of the analysis, facing a quick drop in 2011. On the other hand, the time series of P3 (the light blue one) shows that the hotspots in group 2 do not have relevant crime activity 
until 2013 when they start to present relatively high crime rates.

In order to provide guidelines on why such crime patterns are observed in those locations, we used Physical View to inspect the infrastructure around the hotspots. Figure 37 (4-a) shows some pictures of urban infrastructure around P1 over the years. Notice that until 2011 there was a store with a facade in bad conditions, graffiti, trash bags, and break windows. In 2011, the store was replaced by a bank branch, improving cleanliness and possibly the security in this location. The change might have triggered the radical drop in criminality in P1. Regarding P2 (second row of Figure 37 (4-a)), a bridge was under construction before 2011. Once done, the bridge certainly changed the flow of people in that neighborhood, impacting the criminality rate. Performing the same analysis for P3, we observe a different behavior. For several years there was no crime activity in P3, and after 2013, criminality increased dramatically. Using the Physical View, we extract some images of P3 over the years in order to provide guidance about the reasons for the observed pattern. P3 is the entrance of a bus terminal and in front of a bus station. The bottom row of Figure 37 (4-b) shows some pictures of the of P3 over the years. Before 2013 the bus terminal was under construction, as shown in the first two photos. The bus terminal was inaugurated in 2013. It coincides with the beginning of the increase in crime rates in that location. Contrary to $\mathrm{P} 1$ and $\mathrm{P} 2$, the urban infrastructure might have triggered the criminality in that location.

The three examples show that urban infrastructure can certainly impacts the crime activity. $\mathrm{P} 1$ and $\mathrm{P} 2$ show how completely different infrastructure can positively impact the reduction of crime rates while in $\mathrm{P} 3$ the infrastructure might have triggered crime activities in that location.

\subsection{Evaluation from Experts}

After using our methodology and running a variety of experiments, including the case studies, domain experts have given us the following feedback.

"The methodology and techniques proposed in this paper have allowed a better and accurate understanding of geo-referenced criminal records not yet elucidated by conventional crime analysis resources. First, modeling geocoded crime data within a longitudinal perspective contributes to our understanding of the Spatio-temporal characteristics of criminal victimization, taking a multidisciplinary approach that requires giving proper attention to variation by type of crime, relationships between sets of variables and links between qualitative and quantitative methods. Second, by using robust mathematical and computational mechanisms helps to identify as criminal hotspot not only the most likely dangerous locations, but also the places that may not be receiving enough attention from secure public policies, in spite of frequent criminal activities. Third, spatio-temporal crime patterns are studied by different areas of knowledge, such as social science and data science. Identify similar temporal behavior of hotspots located in different locations leads to thinking the city in its complexity and, at the same time, guide the 
investigation of plausible explanations (demographic profile and urban pattern) for the dynamics of violence, which would be difficult with conventional analysis. Four, CriPAV integrates the proposed techniques in a single tool that enables the analysis of crime geocoded in a street-level of detail, showing the city's singularities and of what these singularities entail. Finally, the results demonstrate the importance of analyzing crime patterns at small scales and has important implications for theoretical development and empirical research.”.

The domain experts discussed the advantages and identified future directions to follow. Besides, they were quite enthusiastic about the identification of similar hotspots: "The identification of specific places with similar criminal behavior could be important to apply similar public policies...”.

\subsection{Discusion and Limitations}

CriPAV has been developed in collaboration with domain experts. As detailed in Section 6.2.2, we have constructed the techniques and tools to attend the experts' demands. However, some limitations and future work have been identified.

- Automatic crime perception on photos. Our approach presents a view called Physical View, which uses google street view to present temporal photo collage in the surroundings of particular hotspots. However, the relationship between physical characteristics and the crime in that location is performed manually. This analysis can be time consuming and exhaustive. An immediate future work is to rely on Image Emotion Recognition to detect the perception of danger in the surrounding infrastructure automatically. Besides, unfortunately, there are no photos before 2010 in google street view.

- Space Discretization. The space discretization employed is the street network. We adopted the network generated by python library osmnx (BOEING, 2017). However, we are aware that there are other representations, some of which with a finer level of detail. Enabling the tool to switch between street network representation is important, and we will address this issue in the future.

- Multiple Data Sources and Scenarios In order to analyze the whole crime story in each location, it is necessary to combine different data sources. For instance, in the case studies, we used information from infrastructure and socioeconomic variables. Given the increasing number of initiatives to make data publicly available, we are considering to incorporate other sources of information with CriPAV. 


\subsection{Final Considerations}

In this chapter, it was presented a visual analytic methodology for street-level crime analysis. This methodology develops two novel techniques: a novel technique based on stochastic matrix to identify hotspots based not only on the intensity but also on the probability; finding similar hotspots, a process for embedding (Hotspot2 $\mathrm{Vec}$ ), clustering, and projecting hotspots based on their temporal behavior. We also developed a visual tool in close collaboration with domain experts. This visual system involves CriPAV's components, and additional visual metaphors to spatio-temporal crime analysis. The proposed techniques are robust and quite effective in identifying important hotspots and reveal important patterns. Our methodology was validated through comparisons and case studies using real data and with feedback from the domain experts. 
CHAPTER

\section{7}

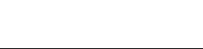

\section{CONCLUSION AND FUTURE WORKS}

In this dissertation, we presented different methodologies that allow a visual Spatiotemporal crime pattern analysis of urban areas considering different characteristics (socioeconomic and infrastructure factors). For that, we have proposed different solutions to tackle the presented problems: (1) we proposed two different methods based on NMF and Stochastic mechanisms for hotspots identification considering not only the intensity of crimes but also the frequency; (2) we have worked with different level of spatial discretization such as census blocks grid and a street-level discretization based on street-network to do more accurate analysis; (3) we developed different visual frameworks to represent spatio-temporal crime patterns based on some automatic mechanisms (i.e., auto-encoders). Each of the proposed approaches has been designed in close collaboration with domain experts and deal simultaneously with multiple requirements. These requirements are translated into analytical tasks that guide the development of the systems. Moreover, the set of case studies, experiments, and experts' feedbacks have shown the usefulness and effectiveness of the proposed methodologies. Supported by the experiments, the results, and the positive feedbacks, it is safe to say the proposed methodologies have the capability and functionalities to analyze successfully different crime patterns in different scenarios.

In Chapter 4, we presented our first approach called CrimAnalyzer, a visual analytics tool to support the analysis of crimes in local regions. CrimAnalyzer solved three major challenges: $i$ ) it is a flexible tool to explore local regions and understand their crime patterns, $i i$ ) it developed a technique based on NMF to identify hotspots that might not be the most prevalent ones in terms of the number of crime events but that are important enough to be investigated, and (iii) it provides multiple resources to help in the understanding of the dynamic of crime patterns over time. Despite satisfying many requirements, the space discretization used in CrimAnalyzer is the census units (See DataSet 3.3.1), and those units do not represent "natural units" of analysis, and the result of certain analyses can change by modifying the aggregation unit.

Subsequently, in order to improve the spatial discretization and to address other domain experts challenges, in Chapter 5, we presented Mirante, a visual analytic tool tailored for 
crime data analysis. Mirante relies on street maps as spatial discretization to identify crime patterns, making it easier to establish relations between crimes and other factors, such as urban infrastructure. Security authorities considered Mirante a valuable tool for different types of analysis in different scenarios. They have pointed out the analysis in a street-level of detail, a functionality they do not have in the crime mapping systems they use to play with.

In Chapter 6, we presented CriPAV, a visual analytic methodology for street-level crime analysis. CriPAV departs from the street-level data modeling used in Mirante. This methodology develops a novel technique based on a stochastic matrix to identify hotspots based not only on the intensity but also on the probability of crimes. Moreover, CriPAV brings another technical contribution to find similar hotspots, based on the embedding (Hotspot2Vec), clustering, and projection of anchor points based on their temporal behavior. We also establish a relation between hotspots and urban factors to reveal phenomena and patterns that were previously unknown by experts in crime analysis.

It is worth mentioning that each proposed methodology was implemented in an original web-based visualization tool developed in close collaboration with domain experts. These visual systems guided case studies to show the effectiveness and usefulness of our proposals in identifying crime-related phenomena. Some case studies were addressed by domain experts to validate some hypothesis with real data in different-sized cities. Moreover, each approach presents experts' feedback, attesting the usefulness of our proposals, highlighting that their current tools do not manage to do the same analysis as presented in this work.

Despite the promising performance of our approaches, there are some limitations. Specifically, our approaches use Google Street View to present temporal photo collage in the surrounding of particular locations. However, the relationship between physical characteristics and the crime in that location is performed manually. This may be solved with Image Emotion Recognition (KO, 2018; COZENS; SAVILLE; HILLIER, 2005b; Arietta et al., 2014) to automatically detect the perception of danger in the surrounding infrastructure.

In our context, considering the geo-referenced data of the crime events and other characteristics of the environment (i.e., street-network topology, traffic data, amenities' localization, socio-economic factors, neighborhood watch, social networks, weather data, etc.), it would be possible to develop compact models to simulate the influence of an establishment (i.e., schools, supermarkets, hospitals, etc.) on the criminality of a region. These environment variables would be important to show us a more accurate reality such as accessibility to certain points, the flow of the people, and localization of crime attractors.

Finally, in this project, we applied and validated our approaches in two different cities. Nevertheless, our methodologies can be extended to multiple scenarios other than crime analysis. For instance, it is possible to implement the system to analyze the geo-referenced data from mobile networks, traffic accidents in a particular location of the city, and epidemic spread. 


\section{BIBLIOGRAPHY}

ADORNO, S. Democracy in progress in contemporary brazil: Corruption, organized crime, violence and new paths to the rule of law. International Journal of Criminology and Sociology, v. 2, p. 409-425, 2013. Citation on page 29.

AKPINAR, E. Using geographic information systems in analysing the pattern of crime incidents and the relationship between landuse and incidents. Middle East Technical University Graduate School of Natural and Applied Sciences, Ankara, Turkey, p. 1-11, 2005. Citation on page 35 .

ALVES, L. G.; RIBEIRO, H. V.; LENZI, E. K.; MENDES, R. S. Distance to the scaling law: a useful approach for unveiling relationships between crime and urban metrics. PloS one, Public Library of Science, v. 8, n. 8, p. 1-8, 2013. Citations on pages 30, 39, 40, and 41.

ALVES, L. G.; RIBEIRO, H. V.; MENDES, R. S. Scaling laws in the dynamics of crime growth rate. Physica A: Statistical Mechanics and its Applications, Elsevier, v. 392, n. 11, p. 2672-2679, 2013. Citations on pages 30, 39, and 40.

ALVES, L. G.; RIBEIRO, H. V.; RODRIGUES, F. A. Crime prediction through urban metrics and statistical learning. Physica A: Statistical Mechanics and its Applications, v. 505, p. 435 - 443, 2018. ISSN 0378-4371. Citation on page 30.

ANDERSON, T. K. Kernel density estimation and k-means clustering to profile road accident hotspots. Accident Analysis \& Prevention, Elsevier, v. 41, n. 3, p. 359-364, 2009. Citation on page 36 .

ANDRESEN, M. A. Mapping crime prevention: What we do and where we need to go. In: Crime Prevention in the 21st Century. [S.1.]: Springer, 2017. p. 113-126. Citation on page 55.

ANSELIN, L. Local Indicators of Spatial Association - LISA. Geographical Analysis, v. 27, p. $93-115,1995$. Citation on page 63.

ANSELIN, L.; BERA, A. K. Introduction to spatial econometrics. Handbook of applied economic statistics, v. 237, 1998. Citation on page 38.

Arietta, S. M.; Efros, A. A.; Ramamoorthi, R.; Agrawala, M. City forensics: Using visual elements to predict non-visual city attributes. IEEE Transactions on Visualization and Computer Graphics, v. 20, n. 12, p. 2624-2633, 2014. Citation on page 118.

BADAWY, M.; CIRELLI, J.; SETYONO, H.; AQLAN, F. Analysis and visualization of city crimes. In: Proceedings of the International Conference on Industrial Engineering and Operations Management. [S.1.: s.n.], 2018. v. 2018, n. SEP, p. 1136-1145. Citation on page 30.

BAILEY, T. C.; GATRELL, A. C. Interactive spatial data analysis. [S.1.]: Longman Scientific \& Technical Essex, 1995. Citations on pages 36 and 40. 
BAK, P.; OMER, I.; SCHRECK, T. Visual analytics of urban environments using high-resolution geographic data. In: Geospatial Thinking. [S.1.]: Springer, 2010. p. 25-42. Citation on page 46.

BALLESTER-RIPOLL, R.; LINDSTROM, P.; PAJAROLA, R. Tthresh: Tensor compression for multidimensional visual data. IEEE Trans. Visual. Comp. Graph., IEEE, 2019. Citation on page 77 .

BENNETT, T.; HOLLOWAY, K.; FARRINGTON, D. P. Does neighborhood watch reduce crime? a systematic review and meta-analysis. Journal of Experimental Criminology, Springer, v. 2, n. 4, p. 437-458, 2006. Citations on pages 40 and 42.

A review of the effectiveness of neighbourhood watch. Security Journal, Springer, v. 22, n. 2, p. 143-155, 2009. Citations on pages 40 and 42.

BERNASCO, W.; BLOCK, R. Robberies in chicago: A block-level analysis of the influence of crime generators, crime attractors, and offender anchor points. Journal of Research in Crime and Delinquency, Sage Publications Sage CA: Los Angeles, CA, v. 48, n. 1, p. 33-57, 2011. Citation on page 79 .

BLOCK, C. R. STAC hot-spot areas: A statistical tool for law enforcement decisions. In: Crime analysis through computer mapping. Washington, DC: Police Executive Research Forum. [S.l.: s.n.], 1995. p. 15-32. Citation on page 36.

BLOCK, R.; BLOCK, C. Space, place and crime: Hot spot areas and hot places of liquor-related crime. Crime Prevention Studies, v. 4, 01 1995. Citation on page 46.

BLOCK, R.; DAVIS, S. The environs of rapid transit stations: A focus for street crime or just another risky place. In: Preventing Mass Transit Crime. [S.1.]: Criminal Justice Press, 1996. Citations on pages 31 and 46.

BOEING, G. OSMnx: new methods for acquiring, constructing, analyzing, and visualizing complex street networks. Computers, Environment and Urban Systems, v. 65, p. 126-139, 2017. ISSN 0198-9715. Citations on pages 82, 108, and 115.

BRAGA, A. A.; HUREAU, D. M.; PAPACHRISTOS, A. V. The relevance of micro places to citywide robbery trends: A longitudinal analysis of robbery incidents at street corners and block faces in boston. Journal of Research in Crime and Delinquency, Sage Publications Sage CA: Los Angeles, CA, v. 48, n. 1, p. 7-32, 2011. Citation on page 100.

BRAGA, A. A.; PAPACHRISTOS, A. V.; HUREAU, D. M. The concentration and stability of gun violence at micro places in boston, 1980-2008. Journal of Quantitative Criminology, Springer, v. 26, n. 1, p. 33-53, 2010. Citation on page 46.

BRANTINGHAM, P. J.; BRANTINGHAM, P. L. Environmental criminology: from theory to urban planning practice. Studies on crime and crime prevention, v. 7, n. 1, p. 31-60, 1998. Citations on pages 30, 31, 39, 96, and 98 .

BREETZKE, G. D.; PEARSON, A. L. The fear factor: Examining the spatial variability of recorded crime on the fear of crime. Applied Geography, Elsevier, v. 46, p. 45-52, 2014. Citation on page 42 . 
BRIMICOMBE, A. J.; RALPHS, M. P.; SAMPSON, A.; TSUI, H. Y. An analysis of the role of neighbourhood ethnic composition in the geographical distribution of racially motivated incidents: Implications for evaluating treatment. British Journal of Criminology, Oxford University Press, v. 41, n. 2, p. 293-308, 2001. Citation on page 40.

BRUNSDON, C.; CORCORAN, J.; HIGGS, G. Visualising space and time in crime patterns: A comparison of methods. Computers, environment and urban systems, Elsevier, v. 31, n. 1, p. 52-75, 2007. Citation on page 45 .

BRUNTON-SMITH, I.; BULLOCK, K. Patterns and drivers of co-production in neighborhood watch in england and wales: From neo-liberalism to new localism. The British Journal of Criminology, Oxford University Press UK, v. 59, n. 1, p. 85-106, 2019. Citation on page 40.

CALDEIRA, T. P.; HOLSTON, J. Democracy and violence in brazil. Comparative studies in society and history, Cambridge University Press, v. 41, n. 4, p. 691-729, 1999. Citation on page 29 .

CALHOUN, C. C.; STOBBART, C. E.; THOMAS, D. M.; VILLARRUBIA, J. A.; BROWN, D. E.; CONKLIN, J. H. Improving crime data sharing and analysis tools for a web-based crime analysis toolkit: Webcat 2.2. In: IEEE. Systems and Information Engineering Design Symposium, 2008. SIEDS 2008. IEEE. [S.1.], 2008. p. 40-45. Citation on page 42.

CAMPELLO, R. J. G. B.; MOULAVI, D.; ZIMEK, A.; SANDER, J. Hierarchical density estimates for data clustering, visualization, and outlier detection. ACM Trans. Knowl. Discov. Data, Association for Computing Machinery, v. 10, n. 1, 2015. ISSN 1556-4681. Citation on page 104 .

CANTER, D.; YOUNGS, D. Crime and society. Contemporary Social Science, Routledge, v. 11, n. 4 , p. 283-288, 2016. Citation on page 30.

CAPLAN, J. M.; KENNEDY, L. W. Risk terrain modeling manual: Theoretical framework and technical steps of spatial risk assessment for crime analysis. [S.1.]: Rutgers Center on Public Security, 2010. Citations on pages 40, 41, and 98.

CHAINEY, S.; RATCLIFFE, J. GIS and Crime Mapping. [S.1.]: Wiley, 2005. Citations on pages $37,40,77$, and 78 .

GIS and crime mapping. [S.1.]: John Wiley \& Sons, 2013. Citations on pages 30, 36, and 40 .

CHAINEY, S.; REID, S.; STUART, N. When is a hotspot a hotspot? a procedure for creating statistically robust hotspot maps of crime. Innovations in GIS, v. 9, p. 21-36, 2002. Citations on pages 36 and 37.

CHAINEY, S.; TOMPSON, L.; UHLIG, S. The utility of hotspot mapping for predicting spatial patterns of crime. Security journal, Springer, v. 21, n. 1-2, p. 4-28, 2008. Citations on pages 30,36 , and 95.

CHEN, H.; ATABAKHSH, H.; PETERSEN, T.; SCHROEDER, J.; BUETOW, T.; CHABOYA, L.; O'TOOLE, C.; CHAU, M.; CUSHNA, T.; CASEY, D.; HUANG, Z. COPLINK: Visualization for crime analysis. In: National Conf. Digital Government Research. [S.1.: s.n.], 2003. Citations on pages 40,42 , and 43 . 
CHEN, H.; ATABAKHSH, H.; TSENG, C.; MARSHALL, B.; KAZA, S.; EGGERS, S.; GOWDA, H.; SHAH, A.; PETERSEN, T.; VIOLETTE, C. Visualization in law enforcement. In: CHI'05 extended abstracts on Human factors in computing systems. [S.1.: s.n.], 2005. p. 1268-1271. Citation on page 30.

CHEN, H.; CHUNG, W.; XU, J. J.; WANG, G.; QIN, Y.; CHAU, M. Crime data mining: A general framework and some examples. Computer, v. 37, n. 4, p. 50-56, 2004. Citation on page 36 .

COZENS, P.; SAVILLE, G.; HILLIER, D. Crime prevention through environmental design (CPTED): A review and modern bibliography. Property Management, v. 23, p. 328-356, 2005. Citations on pages 40 and 41.

COZENS, P. M.; SAVILLE, G.; HILLIER, D. Crime prevention through environmental design (CPTED): a review and modern bibliography. Property management, Emerald Group Publishing Limited, v. 23, n. 5, p. 328-356, 2005. Citation on page 118.

CRAGLIA, M.; HAINING, R.; WILES, P. A comparative evaluation of approaches to urban crime pattern analysis. Urban Studies, Sage Publications Sage UK: London, England, v. 37, n. 4, p. 711-729, 2000. Citations on pages 36 and 40.

DAVIES, T.; JOHNSON, S. D. Examining the relationship between road structure and burglary risk via quantitative network analysis. Journal of Quantitative Criminology, Springer, v. 31, n. 3, p. 481-507, 2015. Citation on page 40.

DAY, P.; BREETZKE, G.; KINGHAM, S.; CAMPBELL, M. Close proximity to alcohol outlets is associated with increased serious violent crime in New Zealand. Australian and New Zealand journal of public health, v. 36, p. 48-54, 2012. Citations on pages 40 and 41.

DEMOTTO, N.; DAVIES, C. A GIS analysis of the relationship between criminal offenses and parks in Kansas City, Kansas. Cartography and Geographic Information Science - CARTOGR GEOGR INF SCI, v. 33, p. 141-157, 2006. Citations on pages 40 and 41.

DERYOL, R.; WILCOX, P.; LOGAN, M.; WOOLDREDGE, J. Crime places in context: An illustration of the multilevel nature of hot spot development. Journal of quantitative criminology, Springer, v. 32, n. 2, p. 305-325, 2016. Citation on page 42.

DULCE, M.; RAMÍREZ-AMAYA, S.; RIASCOS, Á. Efficient allocation of law enforcement resources using predictive police patrolling. arXiv preprint arXiv:1811.12880, 2018. Citations on pages 37 and 40.

EAGLIN, T.; CHO, I.; RIBARSKY, W. Space-time kernel density estimation for real-time interactive visual analytics. In: Proceedings of the 50th Hawaii International Conference on System Sciences. [S.1.: s.n.], 2017. Citation on page 43.

ECK, J.; CHAINEY, S.; CAMERON, J.; WILSON, R. Mapping crime: Understanding hotspots. [S.1.], 2005. Citations on pages 55 and 57.

ECK, J.; WEISBURD, D. L. Crime places in crime theory. Crime and place: Crime prevention studies, v. 4, p. 1-33, 2015. Citations on pages 30, 31, 39, 96, and 98. 
EFTELIOGLU, E.; SHEKHAR, S.; TANG, X. Crime hotspot detection: A computational perspective. In: Improving the Safety and Efficiency of Emergency Services: Emerging Tools and Technologies for First Responders. [S.1.]: IGI Global, 2020. p. 209-238. Citations on pages 30 and 55.

EMIG, M.; HECK, R.; KRAVITZ, M. Crime analysis-a selected bibliography. Washington, DC: US National Criminal Justice Reference Service, 1980. Citation on page 35.

ESTER, M.; KRIEGEL, H.-P.; SANDER, J.; XU, X. A density-based algorithm for discovering clusters in large spatial databases with noise. In: Proceedings of the Second International Conference on Knowledge Discovery and Data Mining. [S.1.]: AAAI Press, 1996. (KDD'96), p. 226-231. Citation on page 104.

FELSON, M.; BOBA, R. L. Crime and everyday life. [S.1.]: Sage, 2010. Citation on page 35.

FENG, Z.; LI, H.; ZENG, W.; YANG, S.-H.; QU, H. Topology density map for urban data visualization and analysis. arXiv preprint arXiv:2007.15828, 2020. Citations on pages 40 and 100 .

FERREIRA, A.; RUBIANO, G.; MOJICA-NAVA, E. Urban security analysis in the city of bogotá using complex networks. In: SPRINGER. International Conference on Complex Systems. [S.1.], 2018. p. 424-438. Citation on page 46.

GAO, R.; TAO, H.; CHEN, H.; WANG, W.; ZHANG, J. Multi-view display coordinated visualization design for crime solving analysis: Vast challenge 2014: Honorable mention for effective use of coordinated visualizations. In: Conf. VAST. [S.1.: s.n.], 2014. p. 321-322. Citations on pages 36 and 42 .

GARCIA-ZANABRIA, G.; GOMEZ-NIETO, E.; SILVEIRA, J.; POCO, J.; NERY, M.; ADORNO, S.; NONATO, L. G. Mirante: A visualization tool for analyzing urban crimes. p. $148-155,2020$. Citations on pages $31,40,42,50,82,84,85$, and 86.

GARCIA-ZANABRIA, G.; RAIMUNDO, M. M.; POCO, J.; NERY, M. B.; SILVA, C. T.; ADORNO, S.; NONATO, L. G. Cripav: Street-level crime patterns analysis and visualization. Submitted: IEEE Transactions on Visualization and Computer Graphics, IEEE, 2020. Citations on pages 32, 40, 42, 50, 96, 99, 100, 102, 103, 106, 110, 112, and 113.

GARCIA-ZANABRIA, G.; SILVEIRA, J. A.; POCO, J.; PAIVA, A.; NERY, M. B.; SILVA, C. T.; ADORNO, S.; NONATO, L. G. Crimanalyzer: Understanding crime patterns in São Paulo. IEEE Transactions on Visualization and Computer Graphics, IEEE, 2019. Citations on pages $30,31,40,42,50,51,59,61,63,64,65,66,70,72,73,74,75,97$, and 98 .

GETIS, A.; ORD, J. K. The analysis of spatial association by use of distance statistics. Geographical Analysis, v. 24, n. 3, p. 189-206, 1992. Citation on page 63.

GODWIN, A.; STASKO, J. Hotsketch: Drawing police patrol routes among spatiotemporal crime hotspots. In: Proceedings of the 50th Hawaii International Conference on System Sciences. [S.1.: s.n.], 2017. Citations on pages 40, 42, 44, and 47.

GOLD, O.; SHARIR, M. Dynamic time warping and geometric edit distance: Breaking the quadratic barrier. ACM Transactions on Algorithms (TALG), ACM New York, NY, USA, v. 14, n. 4, p. 1-17, 2018. Citation on page 104. 
GOMEZ-LIEVANO, A.; YOUN, H.; BETTENCOURT, L. M. The statistics of urban scaling and their connection to Zipf's law. PloS one, Public Library of Science, v. 7, n. 7, p. e40393, 2012. Citations on pages 39 and 40.

GOMEZ-LIEVANO, A.; YOUN, H.; BETTENCOURT, L. M. A. The statistics of urban scaling and their connection to Zipf's law. PLOS ONE, Public Library of Science, v. 7, p. 1-11, 2012. Citation on page 30 .

GOMEZ-NIETO, E.; CASACA, W.; MOTTA, D.; HARTMANN, I.; TAUBIN, G.; NONATO, L. G. Dealing with multiple requirements in geometric arrangements. IEEE transactions on visualization and computer graphics, IEEE, v. 22, n. 3, p. 1223-1235, 2015. Citation on page 108.

GRAAN, J. V. Multi-sector cooperation in preventing crime: the case of a south african neighbourhood watch as an effective crime prevention model. Police Practice and research, Taylor \& Francis, v. 17, n. 2, p. 136-148, 2016. Citations on pages 40 and 42.

GROFF, E. R.; WEISBURD, D.; YANG, S.-M. Is it important to examine crime trends at a local "micro" level?: A longitudinal analysis of street to street variability in crime trajectories. Journal of Quantitative Criminology, Springer, v. 26, n. 1, p. 7-32, 2010. Citations on pages 46, 78, 79 , and 95.

GRUBESIC, T. H.; MURRAY, A. T. Detecting hot spots using cluster analysis and gis. In: Proceedings from the fifth annual international crime mapping research conference. [S.1.: s.n.], 2001. v. 26, p. 1-12. Citations on pages 36, 38, and 40.

GRUBESIC, T. H.; PRIDEMORE, W. A. Alcohol outlets and clusters of violence. International journal of health geographics, Springer, v. 10, n. 1, p. 30, 2011. Citations on pages 40 and 41.

GRUENEWALD, P. J.; FREISTHLER, B.; REMER, L.; LASCALA, E. A.; TRENO, A. Ecological models of alcohol outlets and violent assaults: crime potentials and geospatial analysis. Addiction, v. 101, n. 5, p. 666-677, 2006. Citations on pages 40 and 41.

HART, T.; ZANDBERGEN, P. Kernel density estimation and hotspot mapping. Policing: An International Journal of Police Strategies \& Management, Emerald Group Publishing Limited, 2014. Citation on page 36.

HIRSCHFIELD, A.; BOWERS, K. Mapping and analysing crime data: Lessons from research and practice. [S.1.]: CRC Press, 2014. Citations on pages 36, 37, and 40.

HOJMAN, D. E. Explaining crime in Buenos Aires: the roles of inequality, unemployment, and structural change. Bulletin of Latin American Research, Wiley Online Library, v. 21, n. 1, p. 121-128, 2002. Citations on pages 30, 39, and 40.

Inequality, unemployment and crime in Latin American cities. Crime, Law and Social Change, v. 41, n. 1, p. 33-51, 2004. ISSN 1573-0751. Citations on pages 39, 40, and 41.

HOJMAN, D. E. et al. Unemployment, Inequality and Crime: Latin American Cities under Neoliberal Policies. [S.1.]: Department of Economics and Accounting, University of Liverpool, 2001. Citation on page 30 .

HU, Y.; WANG, F.; GUIN, C.; ZHU, H. A spatio-temporal kernel density estimation framework for predictive crime hotspot mapping and evaluation. Applied geography, Elsevier, v. 99, p. 89-97, 2018. Citations on pages 37, 40, and 45. 
Huang, X.; Zhao, Y.; Ma, C.; Yang, J.; Ye, X.; Zhang, C. Trajgraph: A graph-based visual analytics approach to studying urban network centralities using taxi trajectory data. IEEE Transactions on Visualization and Computer Graphics, v. 22, n. 1, p. 160-169, 2016. Citations on pages 40 and 47.

INGRAM, M. C.; COSTA, M. Marchesini da. A spatial analysis of homicide across Brazil's municipalities. Homicide studies, SAGE Publications Sage CA: Los Angeles, CA, v. 21, n. 2, p. 87-110, 2017. Citation on page 29.

JACOBS, B. A. Robbing drug dealers: Violence beyond the law. [S.1.]: Transaction Publishers, 2000. Citation on page 100.

JEAN, P. Pockets of crime: Broken windows, collective efficacy, and the criminal point of view. Bibliovault OAI Repository, the University of Chicago Press, p. 1-278, 01 2007. Citations on pages 46 and 100 .

JOHNSON, S. D.; BERNASCO, W.; BOWERS, K. J.; ELFFERS, H.; RATCLIFFE, J.; RENGERT, G.; TOWNSLEY, M. Space-time patterns of risk: a cross national assessment of residential burglary victimization. Journal of Quantitative Criminology, Springer, v. 23, n. 3, p. 201-219, 2007. Citation on page 31.

JOHNSON, S. D.; BOWERS, K. J. et al. Stable and fluid hotspots of crime: differentiation and identification. Built Environment, Alexandrine Press, v. 34, n. 1, p. 32-45, 2008. Citations on pages 36 and 40 .

JOIA, P.; COIMBRA, D.; CUMINATO, J. A.; PAULOVICH, F. V.; NONATO, L. G. Local affine multidimensional projection. IEEE Transactions on Visualization and Computer Graphics, IEEE, v. 17, n. 12, p. 2563-2571, 2011. Citation on page 105.

Joshi, A.; Sabitha, A. S.; Choudhury, T. Crime analysis using k-means clustering. In: 2017 3rd International Conference on Computational Intelligence and Networks (CINE). [S.1.: s.n.], 2017. v. 1, n. 1, p. 33-39. Citation on page 30.

JUNIOR, F. C. N.; SILVA, T. L. C. d.; NETO, J. F. d. Q.; MACÊDO, J. A. F. d.; PORCINO, W. C. A novel approach to approximate crime hotspots to the road network. In: Proceedings of the 3rd ACM SIGSPATIAL International Workshop on Prediction of Human Mobility. [S.1.: s.n.], 2019. p. 53-61. Citation on page 40.

KALINIC, M.; KRISP, J. Kernel density estimation (kde) vs. hot-spot analysis detecting criminal hot spots in the city of San Francisco. In: Proceeding of the 21 Conference on GeoInformation Science. [S.1.: s.n.], 2018. v. 1, n. 1. Citations on pages 36 and 37.

KELLY, M. Inequality and crime. Review of economics and Statistics, MIT Press, v. 82, n. 4, p. 530-539, 2000. Citations on pages 30, 39, and 40.

KIM, H.; PARK, H. Sparse non-negative matrix factorizations via alternating non-negativityconstrained least squares for microarray data analysis. Bioinformatics, Oxford University Press, v. 23, n. 12, p. 1495-1502, 2007. Citation on page 63.

KIM, S.; JEONG, S.; WOO, I.; JANG, Y.; MACIEJEWSKI, R.; EBERT, D. S. Data flow analysis and visualization for spatiotemporal statistical data without trajectory information. IEEE transactions on visualization and computer graphics, IEEE, v. 24, n. 3, p. 1287-1300, 2018. Citation on page 42. 
KO, B. C. A brief review of facial emotion recognition based on visual information. sensors, Multidisciplinary Digital Publishing Institute, v. 18, n. 2, p. 401, 2018. Citation on page 118.

KOPPEN, P. van; JANSEN, R. The time to rob: variations in time of number of commercial robberies. Journal of Research in Crime and Delinquency, SAGE Publications, Inc., v. 36, n. 1, p. 7-29, 1999. Citation on page 74 .

KRISHNA, K.; MURTY, M. N. Genetic k-means algorithm. IEEE Transactions on Systems, Man, and Cybernetics, Part B (Cybernetics), IEEE, v. 29, n. 3, p. 433-439, 1999. Citation on page 38 .

LAND, K. C.; DEANE, G. On the large-sample estimation of regression models with spatial-or network-effects terms: A two-stage least squares approach. Sociological methodology, JSTOR, p. 221-248, 1992. Citation on page 38.

LAURITSEN, J. L.; REZEY, M. L.; HEIMER, K. Violence and economic conditions in the united states, 1973-2011: Gender, race, and ethnicity patterns in the national crime victimization survey. Journal of Contemporary Criminal Justice, v. 30, n. 1, p. 7-28, 2014. Citations on pages 30,39 , and 40 .

LAW, S.; NEIRA, M. An unsupervised approach to geographical knowledge discovery using street level and street network images. In: Proceedings of the 3rd ACM SIGSPATIAL International Workshop on AI for Geographic Knowledge Discovery. [S.1.: s.n.], 2019. p. 56-65. Citation on page 46.

LEE, D. D.; SEUNG, H. S. Algorithms for non-negative matrix factorization. In: Advances in Neural Information Processing Systems. [S.1.: s.n.], 2001. p. 556-562. Citations on pages 56 and 60 .

LEVINE, N. CrimeStat: A Spatial Statistical Program for the Analysis of Crime Incidents. 2017. Citations on pages 36 and 39.

LEVITT, S. Alternative strategies for identifying the link between unemployment and crime. Journal of Quantitative Criminology, v. 17, p. 377-390, 2001. Citation on page 30.

LEVITT, S. D. Alternative strategies for identifying the link between unemployment and crime. Journal of quantitative criminology, Springer, v. 17, n. 4, p. 377-390, 2001. Citations on pages 39 and 40 .

LIEBOW, E. Tally's corner: A study of Negro streetcorner men. [S.l.]: Rowman \& Littlefield, 2003. Citation on page 100.

LIVINGSTON, M. A longitudinal analysis of alcohol outlet density and domestic violence. Addiction (Abingdon, England), v. 106, p. 919-25, 2010. Citations on pages 40 and 41.

MACIEJEWSKI, R.; RUDOLPH, S.; HAFEN, R.; ABUSALAH, A.; YAKOUT, M.; OUZZANI, M.; CLEVELAND, W. S.; GRANNIS, S. J.; EBERT, D. S. A visual analytics approach to understanding spatiotemporal hotspots. IEEE Transactions on Visualization and Computer Graphics, IEEE, v. 16, n. 2, p. 205-220, 2009. Citation on page 45.

MALIK, A.; MACIEJEWSKI, R.; COLLINS, T. F.; EBERT, D. S. Visual analytics law enforcement toolkit. In: IEEE. Technologies for Homeland Security (HST), 2010 IEEE International Conference on. [S.1.], 2010. p. 222-228. Citations on pages 40, 42, 43, and 45. 
MALIK, A.; MACIEJEWSKI, R.; TOWERS, S.; MCCULLOUGH, S.; EBERT, D. S. Proactive spatiotemporal resource allocation and predictive visual analytics for community policing and law enforcement. IEEE transactions on visualization and computer graphics, IEEE, v. 20, n. 12, p. 1863-1872, 2014. Citations on pages 40 and 44.

MCCORD, E. S.; RATCLIFFE, J. H. Intensity value analysis and the criminogenic effects of land use features on local crime patterns. Crime Patterns and Analysis, v. 2, n. 1, p. 17-30, 2009. Citations on pages 40 and 41.

MCCULLOCH, J. et al. Criminal justice and political cultures: National and international dimensions of crime control [book review]. Current Issues in Criminal Justice, University of Sydney, Institute of Criminology, v. 17, n. 2, p. 320, 2005. Citations on pages 30, 39, and 95.

MCEWEN, J. T.; TAXMAN, F. S. Applications of computer mapping to police operations. Crime and place, Criminal Justice Press Monsey, New York, v. 4, p. 259-284, 1995. Citation on page 40 .

MCLAFFERTY, S.; WILLIAMSON, D.; MCGUIRE, P. Identifying crime hot spots using kernel smoothing. V. Goldsmith. PO McGuire, JH Mollenkopf and TA Ross Crime Mapping and the Training needs of Law Enforcement, v. 127, 2000. Citations on pages 36 and 40.

MELO, S. N. de; MATIAS, L. F.; ANDRESEN, M. A. Crime concentrations and similarities in spatial crime patterns in a Brazilian context. Applied Geography, Elsevier, v. 62, p. 314-324, 2015. Citation on page 42.

MENCKEN, F. C.; BARNETT, C. Murder, nonnegligent manslaughter, and spatial autocorrelation in mid-south counties. Journal of Quantitative Criminology, Springer, v. 15, n. 4, p. 407-422, 1999. Citations on pages 36, 38, and 40.

MOHD, M. R. S.; HERMAN, S. H.; SHARIF, Z. Application of k-means clustering in hot spot detection for thermal infrared images. In: IEEE. 2017 IEEE Symposium on Computer Applications \& Industrial Electronics (ISCAIE). [S.1.], 2017. p. 107-110. Citations on pages 36,38 , and 40 .

MOHLER, G. O.; SHORT, M. B.; BRANTINGHAM, P. J.; SCHOENBERG, F. P.; TITA, G. E. Self-exciting point process modeling of crime. Journal of the American Statistical Association, Taylor \& Francis, v. 106, n. 493, p. 100-108, 2011. Citation on page 37.

MORAIS, J. D.; SILVA, R. R. D.; ROCHA, E. C. O. Análise multitemporal da ocorrência de queimadas no bioma cerrado no periodo de 2010 a 2013 utilizando tecnologias da geoinformação. 2014. Citation on page 37.

MUNZNER, T. A nested model for visualization design and validation. IEEE TVCG, v. 15, n. 6, p. 921-928, 2009. Citation on page 57.

MURRAY, J.; CERQUEIRA, D. R. de C.; KAHN, T. Crime and violence in Brazil: Systematic review of time trends, prevalence rates and risk factors. Aggression and Violent Behavior, v. 18, n. 5 , p. $471-483$, 2013. ISSN 1359-1789. Citation on page 29.

NAKAYA, T.; YANO, K. Visualising crime clusters in a space-time cube: An exploratory dataanalysis approach using space-time kernel density estimation and scan statistics. Transactions in GIS, Wiley Online Library, v. 14, n. 3, p. 223-239, 2010. Citation on page 45. 
NATH, S. V. Crime pattern detection using data mining. In: IEEE. Web intelligence and intelligent agent technology workshops, 2006. wi-iat 2006 workshops. 2006 ieee/wic/acm international conference on. [S.1.], 2006. p. 41-44. Citation on page 42.

NETO, J. F. de Q.; SANTOS, E.; VIDAL, C. A.; EBERT, D. S. A visual analytics approach to facilitate crime hotspot analysis. Europhysics, v. 39, n. 3, p. 1-13, 2020. Citations on pages 40, $42,43,45$, and 48 .

NETO, J. F. de Q.; SANTOS, E. M. dos; VIDAL, C. A. Mskde-using marching squares to quickly make high quality crime hotspot maps. In: IEEE. 2016 29th SIBGRAPI Conference on Graphics, Patterns and Images (SIBGRAPI). [S.1.], 2016. p. 305-312. Citations on pages $15,37,40$, and 43.

NIE, K.; WANG, Z.; DU, Q.; REN, F.; TIAN, Q. A network-constrained integrated method for detecting spatial cluster and risk location of traffic crash: A case study from wuhan, China. Sustainability, Multidisciplinary Digital Publishing Institute, v. 7, n. 3, p. 2662-2677, 2015. Citations on pages 37 and 40.

NONATO, L. G.; CARMO, F. P.; SILVA, C. T. Glog: Laplacian of gaussian for spatial pattern detection in spatio-temporal data. IEEE Transactions on Visualization and Computer Graphics, IEEE, n. (online first), 2020. Citation on page 30.

OLIVEIRA, M.; BASTOS-FILHO, C.; MENEZES, R. The scaling of crime concentration in cities. PloS one, Public Library of Science, v. 12, n. 8, p. e0183110, 2017. Citations on pages 30, 39, and 40 .

O'MAHONY, M. The relative influence of proximity to fast road infrastructure, accessibility, and deprivation on crime. Journal of Advanced Transportation, Hindawi, v. 2018, 2018. Citation on page 88 .

OpenStreetMap contributors. Planet dump retrieved from https://planet.osm.org 2017. $<$ https://www.openstreetmap.org $>$. Citation on page 82.

ORD, J. K.; GETIS, A. Local spatial autocorrelation statistics: distributional issues and an application. Geographical analysis, Wiley Online Library, v. 27, n. 4, p. 286-306, 1995. Citation on page 63.

OSGOOD, D. W. Poisson-based regression analysis of aggregate crime rates. Journal of quantitative criminology, Springer, v. 16, n. 1, p. 21-43, 2000. Citations on pages 39 and 40.

Statistical models of life events and criminal behavior. In: Handbook of Quantitative Criminology. [S.1.]: Springer, 2010. p. 375-396. Citation on page 36.

OTSU, N. A threshold selection method from gray-level histograms. IEEE Transactions on Systems, Man, and Cybernetics, IEEE, v. 9, n. 1, p. 62-66, 1979. Citation on page 62.

PATTAVINA, A.; BYRNE, J. M.; GARCIA, L. An examination of citizen involvement in crime prevention in high-risk versus low-to moderate-risk neighborhoods. Crime \& Delinquency, Sage Publications Sage CA: Thousand Oaks, CA, v. 52, n. 2, p. 203-231, 2006. Citations on pages 40 and 42 .

POLVI, N.; LOOMAN, T.; HUMPHRIES, C.; PEASE, K. The time course of repeat burglary victimization. The British Journal of Criminology, Oxford University Press, v. 31, n. 4, p. 411-414, 1991. Citation on page 75. 
PORTA, S.; CRUCITTI, P.; LATORA, V. The network analysis of urban streets: A dual approach. Physica A: Statistical Mechanics and its Applications, Elsevier, v. 369, n. 2, p. 853-866, 2006. Citation on page 46.

POVEDA, A. C. Violence and economic development in colombian cities: a dynamic panel data analysis. Journal of international development, Wiley Online Library, v. 24, n. 7, p. 809-827, 2012. Citations on pages 30, 39, and 40.

RATCLIFFE, J. H.; MCCULLAGH, M. J. Hotbeds of crime and the search for spatial accuracy. Journal of geographical systems, Springer, v. 1, n. 4, p. 385-398, 1999. Citations on pages 36 and 40.

RAZIP, A. M.; MALIK, A.; AFZAL, S.; POTRAWSKI, M.; MACIEJEWSKI, R.; JANG, Y.; ELMQVIST, N.; EBERT, D. S. A mobile visual analytics approach for law enforcement situation awareness. In: IEEE. Visualization Symposium (PacificVis), 2014 IEEE Pacific. [S.1.], 2014. p. 169-176. Citations on pages 42 and 44.

REY, S. J.; ANSELIN, L. PySAL: A Python Library of Spatial Analytical Methods. The Review of Regional Studies, v. 37, n. 1, p. 5-27, 2007. Citation on page 64.

ROBINSON, A. I.; CARNES, F.; ORESKOVIC, N. M. Spatial analysis of crime incidence and adolescent physical activity. Preventive medicine, Elsevier, v. 85, p. 74-77, 2016. Citation on page 42 .

S, C.; CHA, S. A survey of binary similarity and distance measures. Journal of Systemics, Cybernetics and Informatics, p. 43-48, 2010. Citation on page 63.

SANDERS, C.; CONDON, C. Crime analysis and cognitive effects: the practice of policing through flows of data. Global Crime, Routledge, v. 18, n. 3, p. 237-255, 2017. Citation on page 30 .

SANTOS, R. B. Crime analysis with crime mapping. [S.1.]: Sage, 2016. Citation on page 36.

SHAO, L.; MITTELSTÄDT, S.; GOLDBLATT, R.; OMER, I.; BAK, P.; SCHRECK, T. Analysis and comparison of feature-based patterns in urban street networks. In: SPRINGER. International Joint Conference on Computer Vision, Imaging and Computer Graphics. [S.1.], 2016. p. 287-309. Citations on pages 40 and 46.

SHIODE, S. Street-level spatial scan statistic and STAC for analysing street crime concentrations. Transactions in GIS, Wiley Online Library, v. 15, n. 3, p. 365-383, 2011. Citations on pages 39 and 40.

SHIODE, S.; SHIODE, N. Network-based space-time search-window technique for hotspot detection of street-level crime incidents. International Journal of Geographical Information Science, Taylor \& Francis, v. 27, n. 5, p. 866-882, 2013. Citations on pages 39 and 40.

SHIODE, S.; SHIODE, N.; BLOCK, R.; BLOCK, C. R. Space-time characteristics of microscale crime occurrences: an application of a network-based space-time search window technique for crime incidents in Chicago. International Journal of Geographical Information Science, Taylor \& Francis, v. 29, n. 5, p. 697-719, 2015. Citations on pages 39 and 40.

SILVA, L. J. S.; FIOL-GONZÁLEZ, S.; ALMEIDA, C. F.; BARBOSA, S. D.; LOPES, H. Crimevis: An interactive visualization system for analyzing crime data in the state of Rio de Janeiro. In: ICEIS (1). [S.1.: s.n.], 2017. p. 193-200. Citations on pages 30, 40, 42, 45, and 47. 
SIRINGI, N.; MALA, S.; RAWAT, A. Study of k-means clustering algorithm for identification of dengue fever hotspots. In: ICDSMLA 2019. [S.1.]: Springer, 2020. p. 51-61. Citations on pages 38 and 40 .

SOLTANI, A.; ASKARI, S. Exploring spatial autocorrelation of traffic crashes based on severity. Injury, Elsevier, v. 48, n. 3, p. 637-647, 2017. Citation on page 40.

SPADON, G.; SCABORA, L. C.; ARAUJO, M. V.; OLIVEIR, P. H.; MACHADO, B. B.; SOUSA, E. P.; TRAINA, C.; RODRIGUES, J. F. Complex-network tools to understand the behavior of criminality in urban areas. In: Information Technology-New Generations. [S.1.]: Springer, 2018. p. 493-500. Citation on page 46.

SPICER, V.; SONG, J.; BRANTINGHAM, P.; PARK, A.; ANDRESEN, M. A. Street profile analysis: A new method for mapping crime on major roadways. Applied Geography, Elsevier, v. 69 , p. $65-74,2016$. Citations on pages 40 and 42.

SZABO, F. The Linear Algebra Survival Guide: Illustrated with Mathematics. [S.1.]: Academic Press, 2015. Citation on page 101.

ULLAH, A. Handbook of applied economic statistics. [S.1.]: CRC Press, 1998. Citation on page 38 .

VALDIVIA, P.; DIAS, F.; PETRONETTO, F.; SILVA, C. T.; NONATO, L. G. Wavelet-based visualization of time-varying data on graphs. In: IEEE Visual Analytics Science and Technology (VAST). [S.1.: s.n.], 2015. p. 1-8. Citation on page 60.

VASCONCELOS, V. V.; PINHO, C. M. de D. Multivariate geovisualization of dengue, zika and chikungunya cases in BRAZIL: A didactic experience. Hygeia, v. 13, n. 25, p. 91-106, 2017. Citation on page 37 .

VITO, G. F.; BLANKENSHIP, M. B.; KUNSELMAN, J. C.; SURESH, G. Statistical analysis in criminal justice and criminology: A user's guide. [S.1.]: Waveland Press, 2020. Citation on page 30 .

WALT, S. van der; SCHÖNBERGER, J. L.; Nunez-Iglesias, J.; BOULOGNE, F.; WARNER, J. D.; YAGER, N.; GOUILLART, E.; YU, T.; CONTRIBUTORS the scikit-image. scikit-image: image processing in Python. PeerJ, v. 2, p. e453, 2014. ISSN 2167-8359. Citation on page 109.

WANG, D.; DING, W.; LO, H.; MORABITO, M.; CHEN, P.; SALAZAR, J.; STEPINSKI, T. Understanding the spatial distribution of crime based on its related variables using geospatial discriminative patterns. Computers, Environment and Urban Systems, Elsevier, v. 39, p. 93-106, 2013. Citations on pages 15, 30, 36, 40, 42, 43, 55, and 95.

WANG, T.; RUDIN, C.; WAGNER, D.; SEVIERI, R. Learning to detect patterns of crime. In: Machine Learning and Knowledge Discovery in Databases. [S.1.: s.n.], 2013. p. 515-530. Citation on page 36 .

Wang, Z.; Ye, T.; Lu, M.; Yuan, X.; Qu, H.; Yuan, J.; Wu, Q. Visual exploration of sparse traffic trajectory data. IEEE Transactions on Visualization and Computer Graphics, v. 20, n. 12, p. 1813-1822, 2014. Citation on page 47.

WEISBURD, D.; BUSHWAY, S.; LUM, C.; YANG, S.-M. Trajectories of crime at places: A longitudinal study of street segments in the city of Seattle. Criminology, Wiley Online Library, v. 42, n. 2, p. 283-322, 2004. Citation on page 79. 
WEISBURD, D.; MORRIS, N. A.; GROFF, E. R. Hot spots of juvenile crime: A longitudinal study of arrest incidents at street segments in seattle, washington. Journal of Quantitative Criminology, Springer, v. 25, n. 4, p. 443, 2009. Citations on pages 46, 78, and 95.

WILLIAMS, S.; GALSTER, G.; VERMA, N. Home foreclosures and neighborhood crime dynamics. Housing Studies, Taylor \& Francis, v. 29, n. 3, p. 380-406, 2014. Citation on page 97.

WOODY, C. These were the 50 most violent cities in the world in 2018 . 2018. Available: <web.archive.org/web/20200402213800/https://www.businessinsider.com/ most-violent-cities-in-the-world-in-2018-2019-3>. Citation on page 29.

XIANG, Y.; CHAU, M.; ATABAKHSH, H.; CHEN, H. Visualizing criminal relationships: Comparison of a hyperbolic tree and a hierarchical list. Decision Support Systems, Elsevier, v. 41, n. 1, p. 69-83, 2005. Citation on page 44.

XU, J.; CHEN, H. Criminal network analysis and visualization. Commun. ACM, v. 48, n. 6, p. 100-107, 2005. Citation on page 36.

Yadav, S.; Timbadia, M.; Yadav, A.; Vishwakarma, R.; Yadav, N. Crime pattern detection, analysis prediction. In: 2017 International conference of Electronics, Communication and Aerospace Technology (ICECA). [S.1.: s.n.], 2017. v. 1, p. 225-230. Citations on pages 30 and 36.

YE, N.; WANG, B.; KITA, M.; XIE, M.; CAI, W. Urban commerce distribution analysis based on street view and deep learning. IEEE Access, IEEE, v. 7, p. 162841-162849, 2019. Citation on page 46.

YE, X.; XU, X.; LEE, J.; ZHU, X.; WU, L. Space-time interaction of residential burglaries in wuhan, China. Applied Geography, v. 60, p. 210 - 216, 2015. Citation on page 42.

YING, Z. Analysis of crime factors correlation based on data mining technology. In: Int. Conf. Robots Intel. Sys. (ICRIS). [S.1.: s.n.], 2016. p. 103-106. Citation on page 36.

Zeng, W.; Ye, Y. Vitalvizor: A visual analytics system for studying urban vitality. IEEE Computer Graphics and Applications, v. 38, n. 5, p. 38-53, 2018. Citations on pages 40 and 47.

ZHOU, G.; LIN, J.; ZHENG, W. A web-based geographical information system for crime mapping and decision support. In: IEEE. Computational Problem-Solving (ICCP), 2012 International Conference on. [S.1.], 2012. p. 147-150. Citations on pages 40, 44, and 46.

ZITNIK, M.; ZUPAN, B. Nimfa: A Python Library for Nonnegative Matrix Factorization. Journal of Machine Learning Research, v. 13, p. 849-853, 2012. Citation on page 66. 

APPENDIX

\section{A}

LAYOUT ARRANGEMENT

\section{A.1 Layout Arrangement of Between-Group Chart}

Detailing some technical aspects of such visualization, we want to observe a crime time series representation of each cluster. To do that, we want to show $C$ time-series representation of the clusters in a screen of width of $W$ and height of $H$. The representations must have a size proportional to its clusters size $a_{i}, \forall i \in\{1, \ldots, C\}$ and respect a aspect ratio of $r$. The final requirement reside on arranging such clusters similarly to the projected in the screen where the clusters have its center on $x_{i}^{c}, y_{i}^{c}, \forall i \in\{1, \ldots, C\}$.

Relying on a mixed-integer model we created the following variables: $x_{i}, y_{i}, \forall i \in$ $\{1, \ldots, C\}$ are the localization of the center of the box; $r_{i, j}, \forall i \in\{1, \ldots, C\}, \forall j \in\{1, \ldots, C\}$ shows if there is an intersection of boxes $i$ and $j$ in a single dimension - this variable, with help of some constraints, will forbid having intersections on both dimensions. The variable $\delta$ it is a multiplier of the size of the box - worth mentioning that the height of a box $i$ is given by $\delta a_{i}$, and its width is $r \delta a_{i}$; Given that, we can formulate a set of constraints:

Constraints to calculate the $l_{1}$ distance $d_{i}$ from the center of the box to the reference point:

$$
\begin{array}{r}
\left(x_{i}-x_{i}^{c}\right)+\left(y_{i}-y_{i}^{c}\right) \leq d_{i}, \forall i \in\{1, \ldots, C\} \\
\left(x_{i}-x_{i}^{c}\right)-\left(y_{i}-y_{i}^{c}\right) \leq d_{i}, \forall i \in\{1, \ldots, C\} \\
-\left(x_{i}-x_{i}^{c}\right)+\left(y_{i}-y_{i}^{c}\right) \leq d_{i}, \forall i \in\{1, \ldots, C\} \\
-\left(x_{i}-x_{i}^{c}\right)-\left(y_{i}-y_{i}^{c}\right) \leq d_{i}, \forall i \in\{1, \ldots, C\}
\end{array}
$$


Constraints to avoid intersections:

$$
\begin{aligned}
& x_{i}-x_{j}>=r \delta \frac{a_{i}+a_{j}}{2}-W r_{i, j}, \forall i \in\{1, \ldots, C\}, \\
& \forall j \in\{i+1, \ldots, C\} \\
& y_{j}-y_{i}>=\delta \frac{a_{i}+a_{j}}{2}-H\left(1-r_{i, j}\right), \forall i \in\{1, \ldots, C\}, \\
& \forall j \in\{i+1, \ldots, C\}
\end{aligned}
$$

Constraints to respect the limits of the window:

$$
\begin{array}{r}
x_{i}-r \delta a_{i} / 2 \geq 0, \forall i \in\{1, \ldots, C\} \\
x_{i}+r \delta a_{i} / 2 \leq W, \forall i \in\{1, \ldots, C\} \\
y_{i}-\delta a_{i} / 2 \geq 0, \forall i \in\{1, \ldots, C\} \\
y_{i}-\delta a_{i} / 2 \leq H, \forall i \in\{1, \ldots, C\}
\end{array}
$$

Now it is possible to define the objective functions of the problem:

- Function that increase the screen usage by the boxes in the screen: $f_{1}=\left(\delta^{\max }-\delta\right) / \delta^{\max }-$ $\underline{f}_{1}$, where $\delta^{\max }=\sqrt{\frac{W H}{r \sum a_{i}^{2}}}$, and $\underline{f}_{1}$ is a relaxation of the objective (e.g. if $\underline{f}_{1}=0.1$ the optimization stops at $10 \%$ of $\delta^{\max }$ ).

- Function that makes the boxes as close as possible to the projection: $f_{2}=\frac{\sum d_{i}}{W H N}-f_{2}$, where $\underline{f}_{2}$ is a relaxation of the objective (e.g. if $\underline{f}_{2}=0.1$ the optimization stops at $10 \%$ of $W H N$.

To improve the speed of optimizing the layout arrangement, we make the optimization in two steps: first we only optimize $f_{1}$ with $\underline{f}_{1}=0.2$, finding the optimal values $f_{1} *$ and $f_{2} *$ and then optimize $f_{1}+f_{2}$ constrained by $f_{1} \leq f_{1} *$ and $f_{2} \leq f_{2} *$ improving the optimal value of $f_{2}$ without losing the occupation promoted by optimizing $f_{1}$ ). 


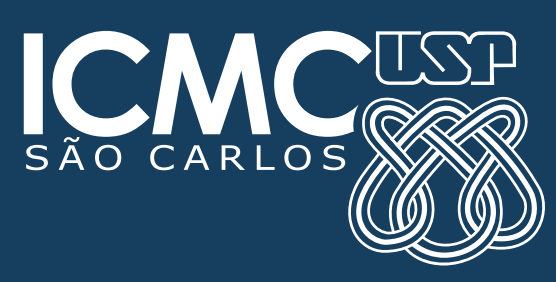

